\title{
Numerical Modelling of Shock Wave Boundary Layer Interactions in Aero-engine Intakes at Incidence
}

\author{
Hardeep S Kalsi \\ Supervisor: Prof. Paul G Tucker \\ Department of Engineering \\ University of Cambridge
}

This thesis is submitted for the degree of

Doctor of Philosophy 



\title{
Numerical Modelling of Shock Wave Boundary Layer Interactions in Aero-engine Intakes at Incidence
}

\author{
Hardeep S Kalsi \\ Supervisor: Prof. Paul G Tucker
}

\begin{abstract}
Aero-engine intakes play a critical role in the performance of modern high-bypass turbofan engines. It is their function to provide uniformly distributed, steady air flow to the engine fan face under a variety of flow conditions. However, during situations of high incidence, high curvature of the intake lip can accelerate flow to supersonic speeds, terminating with a shock wave. This produces undesirable shock wave boundary layer interactions (SWBLIs).

Reynolds-Averaged Navier Stokes (RANS) turbulence models have been shown to be insensitive to the effects of boundary layer relaminarisation present in these highlyaccelerated flows. Further, downstream of the SWBLI, RANS methods fail to capture the distorted flow that propagates towards the engine fan face.

The present work describes simulations of a novel experimental intake rig model that replicates the key physics found in a real intake- namely acceleration, shock and SWBLI. The model is a simple geometric configuration resembling a lower intake lip at incidence. Simulations are carried out at two angles of attack, $\alpha=23^{\circ}$ and $\alpha=25^{\circ}$, with the more aggressive $\alpha=25^{\circ}$ possessing a high degree of shock oscillation.

RANS, Large Eddy Simulations (LES) and hybrid RANS-LES are carried out in this work. Modifications to the one-equation Spalart-Allmaras (SA) RANS turbulence model are proposed to account for the effects of re-laminarisation and curvature. The simulation methods are validated against two canonical test cases. The first is a subsonic hump model where RANS modifications give a noticeable improvement in surface pressure predictions, even for this mild acceleration case. However, RANS is shown to over-predict the separation size. LES performs much better here, as long as the Smagorinsky-Lilly SGS model is not used. The $\sigma$-SGS model is found to perform best, and is used to run a hybrid RANS-LES that predicts a separation bubble size within $4 \%$ of LES.
\end{abstract}

The second canonical test case is a transonic hump that features a normal shock- 
wave and SWBLI. RANS performs well here, predicting shock location, surface pressure and separation with good agreement with experimental measurements. Hybrid RANS-LES also performs well, but predicts a shock downstream of that measured by experiment. The use of an improved shock sensor here is able to maintain solution accuracy.

Simulations of the intake rig are then run. RANS modifications provide a significant improvement in prediction of the shock location and lip surface pressure compared to the standard SA model. However, RANS models fail to reproduce the post shock interaction flow well, giving incorrect shape of the flow distortion. Further, RANS is inherently unable to capture the unsteady shock oscillations and related flow features.

LES and hybrid RANS-LES predict the shock location and SWBLI well, with the downstream flow distortion also in very good agreement with experimental measurements. LES and hybrid RANS-LES are able to reproduce the time averaged smearing of the shock which RANS cannot. However, shock oscillations in the $\alpha=25^{\circ}$ case present a particular challenge for costly LES, requiring long simulation time to obtain time averaged flow statistics. Hybrid RANS-LES offers a significant saving in computational expense, costing approximately $20 \%$ of LES. The work proposes recommendations for simulation strategy for intakes at incidence based on computational cost and performance of simulation methods. 
To my family 



\section{Declaration}

This dissertation is the result of my own work and includes nothing which is the outcome of work done in collaboration except as declared in the Preface and specified in the text. It is not substantially the same as any that I have submitted, or, is being concurrently submitted for a degree or diploma or other qualification at the University of Cambridge or any other University or similar institution except as declared in the Preface and specified in the text. I further state that no substantial part of my dissertation has already been submitted, or, is being concurrently submitted for any such degree, diploma or other qualification at the University of Cambridge or any other University or similar institution except as declared in the Preface and specified in the text.

This thesis contains approximately 37,000 words and 70 figures. It does not exceed the prescribed word limit for the relevant Degree Committee.

Hardeep S Kalsi

Supervisor: Prof. Paul G Tucker

October 2018 



\section{Acknowledgements}

This $\mathrm{PhD}$ would not have been possible without the support of many people. First I would like to sincerely thank my project supervisor, Prof. Paul G Tucker. His invaluable guidance, technical knowledge and enthusiasm during my $\mathrm{PhD}$ has made it a thoroughly enjoyable experience. I would also like to thank my project advisor, Prof. Holger Babinsky, who stepped in during the later stages of my project and helped in the preparation of this thesis.

I would also like to thank Holger and his PhD students Tafara Makuni and Andrea Coschignano who carried out the experimental effort that partnered this work. Being able to physically see the rig I was simulating in action and being able to request specific measurements has added an extra dimension to this project that few are lucky to have.

Next i would like to thank the Cambridge CFD group, whose expertise has greatly helped during challenging times of the project. I am grateful to Rob Watson, Jiahuan Cui, Ashley Scillitoe, Andrea Masi, Bryn Ubald and James Tyack for valuable discussions on CFD methods and the HYDRA CFD code. I would specifically like to thank Rao Vadlamani for the Darwin HPC compute time that enabled the majority of the LES simulations in this project, and also Iffi Nakavi, who generated turbulent boundary layers used for the canonical flow cases.

This project is funded by the European Physical Sciences Research Council (EPSRC) and Rolls-Royce, which is gratefully acknowledged. I would like to thank Chris Sheaf and Kevin Menzies from the installations team at Roll-Royce, whose discussions on the ultimate application of my research has always been a great motivator for me.

Finally, I would like to thank my family, without whom i could not have achieved so much. To my Dad, Mum, Sister, Grandma and late Grandad - who sadly passed away two months before the submission of this thesis- I am deeply grateful for your unconditional love, encouragement and support. 



\section{Table of contents}

Table of contents $\quad$ xi

List of figures $\quad$ xiii

List of tables $\quad$ xvii

1 Introduction $\quad 1$

1.1 Motivations ........................ 1

1.2 Intakes at Incidence . . . . . . . . . . . . . . . . . . . 3

1.3 Computational Fluid Dynamics (CFD) . . . . . . . . . . . . 4

1.4 Aims and Research Objectives . . . . . . . . . . . . . . 6

2 Literature Review $\quad 7$

2.1 Introduction . . . . . . . . . . . . . . . . . 7

2.2 Aero-engine Crosswind Flows _ . . . . . . . . . . . . . 7

2.3 Shock Wave Boundary Layer Interactions (SWBLIs) . . . . . . . . . . . 19

2.3.1 CFD of SWBLIs . . . . . . . . . . . . . . . . 22

2.3.2 Transitional SWBLIs . . . . . . . . . . . . . . 25

2.4 Summary . . . . . . . . . . . . . . . . . . . . 27

2.5 Project Strategy . . . . . . . . . . . . . . . . . . . . . . 28

3 Numerical Methods $\quad 29$

3.1 Turbulence Treatments . . . . . . . . . . . . . . . . . . . . . . 29

3.2 Reynolds-Averaged Navier-Stokes . . . . . . . . . . . . . . . 30

3.2 .1 SA Model . . . . . . . . . . . . . . . . . . . . 32

3.3 Large Eddy Simulations . . . . . . . . . . . . . . . . . 35

3.3.1 Smagorinsky-Lilly Model . . . . . . . . . . . . . . . 36

3.3.2 WALE Model . . . . . . . . . . . . . . . . . . . 37

3.3.3 Sigma Model . . . . . . . . . . . . . . . . . 38 
3.4 Hybrid RANS-LES . . . . . . . . . . . . . . . . . . . . . 38

3.5 HYDRA . . . . . . . . . . . . . . . . . 39

3.5.1 Spacial Discretisation . . . . . . . . . . . . . . 39

3.5.2 Ducros Sensor . . . . . . . . . . . . . . . . . . 42

3.6 Turbulent Boundary Layers . . . . . . . . . . . . . . . . . . . . . . 43

4 Canonical Test Case 1: NASA Hump 45

4.1 Case Details . . . . . . . . . . . . . . . . . . . . . . . 45

4.2 Computational Setup . . . . . . . . . . . . . . . . . . . 47

4.3 Results . . . . . . . . . . . . . . . . . . 50

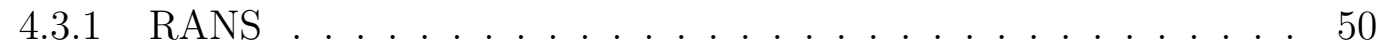

4.3.2 LES and Hybrid RANS-LES . . . . . . . . . . . . . . . . 54

4.4 Conclusions . . . . . . . . . . . . . . . . . . 62

5 Canonical Test Case 2: Axisymmetric Hump 63

5.1 Case Details . . . . . . . . . . . . . . . . . . . . . 64

5.2 Computational Setup . . . . . . . . . . . . . . . . . 65

5.3 Results . . . . . . . . . . . . . . . . . . . 68

5.4 Conclusions . . . . . . . . . . . . . . . . . . . . 74

6 Intake Rig Model $\quad 77$

6.1 Introduction . . . . . . . . . . . . . . . . . . . 77

6.2 Case details . . . . . . . . . . . . . . . . . . . . . . . . . . . . . . 78

6.3 Computational Setup . . . . . . . . . . . . . . . . 81

6.4 Results . . . . . . . . . . . . . . . . . . . . 84

6.4.1 $\alpha=23^{\circ}$ Case . . . . . . . . . . . . . . . . . 84

$6.4 .2 \alpha=25^{\circ}$ Case . . . . . . . . . . . . . . . . . 89

6.5 Conclusion . . . . . . . . . . . . . . . . . . . . 97

7 Concluding Remarks and Recommendations for Future Work 101

7.1 Concluding Remarks . . . . . . . . . . . . . . . . . . . . . . 101

7.2 Recommendations for Future Work . . . . . . . . . . . . . . . . . 102

$\begin{array}{ll}\text { References } & 105\end{array}$ 


\section{List of figures}

1.1 Rolls-Royce Trent-700 at take-off showing the intake section, fan and nacelle . . . . . . . . . . . . . . . . . 1

1.2 Flow features of intakes at incidence . . . . . . . . . . . . . 3

1.3 Hierarchy of CFD methods . . . . . . . . . . . . . . . 5

2.1 Key flow features of intakes in 90 degree crosswind flow . . . . . . . . 8

2.2 Variation of shape factor with acceleration parameter in accelerating flow in a nozzle from Oriji and Tucker [1] . . . . . . . . . . . . . . . 10

2.3 Intake rig case details and results by Oriji and Tucker [1] for pure crosswind flow, with the modified (3-component) SA model matching much closer to experiment . . . . . . . . . . . . . . . . 12

2.4 Hybrid RANS-LES results of crosswind flow over an intake lip by Oriji and Tucker [2] . . . . . . . . . . . . . . . . . . . . . . . . 14

2.5 Flow around an intake under crosswind using LES from Vadlamani and Tucker $[3] \ldots \ldots \ldots \ldots$

2.6 Turbulent flow structures around an intake model by Hall and Hynes [4] 17

2.7 Contours of loss coefficient at the fan face with the intake flow attached and separated from Hall and Hynes [5] . . . . . . . . . . . . . . 18

2.8 3D Intake geometry and crosswind flow separation over an intake lip by Colin et al. [6] . . . . . . . . . . . . . . . . . . . 18

2.9 Shock induced flow separation over a transonic aerofoil from Delery [7] 19

2.10 Typical SWBLIs from [8]. (a) Transonic normal shock (b) Oblique shock (c) supersonic flow over a compression ramp . . . . . . . . . 20

2.11 Typical experimental test cases for the study of SWBLIs from [9] . . . 21

2.12 Comparisons between DNS and experimental flow fields of the shock over a compression ramp by Adams [10] . . . . . . . . . . . 23

2.13 Mach number distributions for a transonic aerofoil under free and fixed transition, and at two shock locations from Becker at al. [11] . . . . . 26 
3.1 HYDRA's dual median control volume around an arbitrary grid node . 40

3.2 Schematic showing the collection of TBL data using the Lund recycling/rescaling technique $[12] \ldots \ldots$. . . . . . . . . . . . 43

3.3 TBL data generated for $R e_{\theta}=8000$, coloured by contours of velocity magnitude . . . . . . . . . . . . . . . . . . . . 44 44

3.4 Mean velocity and Reynolds stress profiles of the two TBLs generated using the Lund recycling method. Lines: Lund recycling TBL data, Symbols: reference LES data at $R e_{\theta}=8300$ from Eitel-Amor et al. [13]

4.1 NASA hump model showing the experimental features from [14] . . . . 46

4.2 Schematic of the NASA hump geometry normalised by the hump chord length C . . . . . . . . . . . . . . . . . . . . . 44 46

4.3 Two-dimensional slice of the computational grid and boundary conditions used for LES of the NASA hump. Every 8th point shown in the streamwise direction and every 4th point shown in the wall normal direction for clarity. Note the contour on the top wall to account for endplate blockage. . . . . . . . . . . . . . . . . . . . . . 47

4.4 Boundary layer velocity profile at $x / c=-2.14 C$ obtained from RANSSA compared to experimental measurements. . . . . . . . . . . . . 49

$4.5 C_{P}$ distribution over the NASA hump using RANS . . . . . . . . . . 51

$4.6 \quad K_{S}$ distribution over the NASA hump forebody . . . . . . . . . . . . 52

4.7 Linear ramp function used to boost production in the separated region $\quad 52$

4.8 Streamwise velocity profiles located downstream of the separation compared with experimental measurements for RANS . . . . . . . . . . . . 53

4.9 Instantaneous Q-criterion contours coloured by velocity over the NASA hump using LES with the $\sigma$-SGS model. The flow direction is left to right. . . . . . . . . . . . . . . . . . 54

$4.10 C_{P}$ distribution over the NASA hump . . . . . . . . . . . . . . 55

4.11 Time averaged axial velocity contours downstream of separation from CFD compared to a plane. Flow reattachment locations are marked $x_{r}$. The vertical dashed line shows the experimental reattachment point . . 57

4.12 Streamwise velocity profiles located downstream of the separation compared with experimental measurements for LES and hybrid RANS-LES

4.13 Reynolds stress profiles located downstream of the separation compared with experimental measurements for LES and hybrid RANS-LES . . .

4.14 Time averaged contours of resolved turbulent kinetic energy (TKE) over the NASA hump for the three LES SGS models tested 
4.15 Turbulent kinetic energy profiles upstream of separation on the hump surface for LES . . . . . . . . . . . . . . . . . . . . 61

5.1 Axisymmetric hump model showing the main flow features . . . . . . . 64

5.2 Schematic of the transonic hump geometry normalised by the hump chord length c . . . . . . . . . . . . . . . . . . 6 65

5.3 Two-dimensional slice of the computational grid and boundary conditions used for hybrid RANS-LES of the transonic hump. Every 8th point shown in the streamwise direction and every 4th point shown in the wall normal direction for clarity. . . . . . . . . . . . . . . 65

5.4 Computational domain used for hybrid RANS-LES simulations of the transonic hump . . . . . . . . . . . . . . . . 6 66

5.5 Boundary layer velocity profile at $x / c=-0.25 C$ obtained from RANSSA compared to experimental measurements. . . . . . . . . . . 67

5.6 Mach number contours of flow over the transonic hump . . . . . . . . 68

5.7 Instantaneous Q-criterion contours coloured by velocity over that transonic hump using hybrid RANS-LES with the $\sigma$-SGS model. The flow direction is left to right. Note the grey iso-surface showing the boundary of the supersonic region $(\mathrm{M}=1) \ldots \ldots \ldots$

5.8 Comparisons of the classical Jameson shock sensor and the improved Ducros sensor . . . . . . . . . . . . . . . . . 70

$5.9 C_{p}$ distribution over the transonic hump . . . . . . . . . . . . 71

5.10 Streamwise velocity profiles located downstream of the shock separation compared with experimental measurements for RANS and hybrid RANS-LES

5.11 Turbulent kinetic energy and Reynolds stress profiles located downstream of the shock separation compared with experimental measurements for hybrid RANS-LES . . . . . . . . . . . . . 73

5.12 Reynolds stress profiles at a fixed height of $0.01 \mathrm{c}$ above the hump surface 74

6.1 Simplification of a real intake to a two dimensional aerofoil. Figure

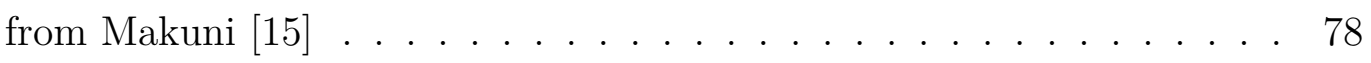

6.2 Schematic of the intake rig working section and key components . . . . 79

6.3 Photos of the Intake rig and aerofoil . . . . . . . . . . . . . . . . 80

6.4 Processed time-averaged surface pressure data captured experimentally for both $\alpha=23^{\circ}$ and $\alpha=25^{\circ}$ cases using PSP. Flow direction is left to right. . . . . . . . . . . . . . . . . . 81 
6.5 Two-dimensional slice of the computational grid and boundary conditions used for LES of the intake rig at $\alpha=25^{\circ}$. Every 8th point shown for clarity. Inset: close up of the grid around the lip. Note the blue lines showing the O-grid around the aerofoil. . . . . . . . . . 82

6.6 Static pressure distribution around the aerofoil using the RANS SA model 85

6.7 Effects of varying underside blockage on the lip flow. Top: CFD using the RANS SA model, bottom: experimental schlieren images . . . . . . 85

6.8 Comparison of flow features between experimental and CFD results for

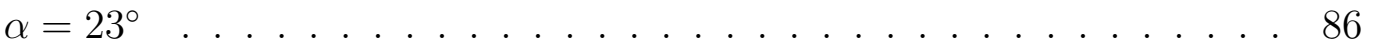

6.9 Instantaneous contours of Q-criterion coloured by velocity for hybrid RANS-LES at $\alpha=23^{\circ}$. Note the grey iso-surface showing the boundary of the supersonic region $(\mathrm{M}=1) \ldots \ldots \ldots$. . . . . . . . . 87

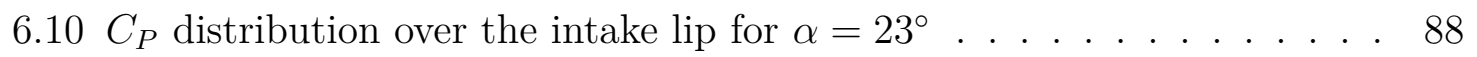

6.11 Velocity profiles at 3 locations normal to the intake aerofoil surface for $\alpha=23^{\circ} \ldots \ldots \ldots \ldots \ldots \ldots \ldots \ldots$

6.12 Skin friction coefficient distribution over the intake aerofoil lip for $\alpha=23^{\circ} 89$

6.13 Instantaneous contours of Q-criterion coloured by velocity for LES at $\alpha=25^{\circ}$. Note the grey iso-surface showing the boundary of the supersonic region $(\mathrm{M}=1) \ldots \ldots . \ldots . \ldots . \ldots 90$

6.14 Comparisson of the classical Jameson shock sensor and the improved Ducros sensor . . . . . . . . . . . . . . . . . . . . 91

$6.15 C_{P}$ distribution over the intake lip for $\alpha=25^{\circ} \ldots \ldots$. . . . . . . 92

6.16 Time-averaged contours of Mach number over the aerofoil lip region for $\alpha=25^{\circ}$

6.17 Velocity profiles at one location normal to the intake aerofoil surface

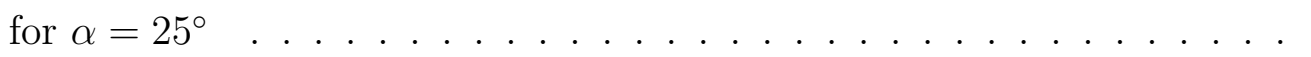

6.18 Velocity and turbulence intensity profiles at 5 locations over the intake aerofoil lip for $\alpha=25^{\circ} \ldots \ldots$. . . . . . . . . . . . . 95

6.19 Skin friction coefficient distribution over the intake aerofoil lip for $\alpha=25^{\circ} 96$

6.20 Instantaneous contours of Q-criterion coloured by velocity using LES for $\alpha=25^{\circ}$ at 3 time instances. Note the grey iso-surface showing the boundary of the supersonic region $(\mathrm{M}=1) . x_{t}$ and $x_{s}$ mark the onset of transition and shock location respectively . . . . . . . . . . . . . . 98

6.21 Comparisson of secondary flow features between CFD and exprimental schlieren image for $\alpha=25^{\circ} \ldots \ldots . \ldots . \ldots . . \ldots 99$

7.1 Recommended zonalisation strategies for future intake rig simulations . 103 


\section{List of tables}

2.1 Grid requirements for different eddy resolving methods from Tucker [16]. $\Delta x^{+}, \Delta y^{+}$and $\Delta z^{+}$are the recommended grid spacings in the streamwise, wall normal and cross stream directions respectively. . . . . 13

4.1 Separation, reattachment and bubble size over the NASA hump . . . . 56

5.1 Separation, reattachment and bubble size over the transonic hump . . . 71

6.1 Separation, reattachment and bubble size over the intake aerofoil lip for

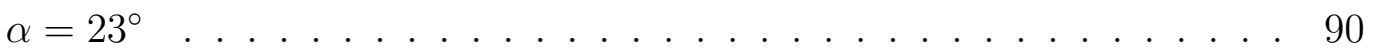

6.2 Separation, reattachment and bubble size over the intake aerofoil lip for

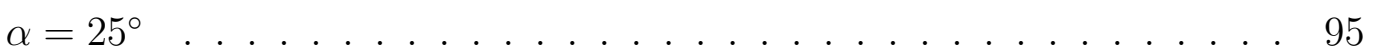





\title{
Nomenclature
}

\author{
Abbreviations \\ BL Boundary Layer \\ CFD Computational Fluid Dynamics \\ CFL Courant Friedrichs Lewy condition \\ DES Detached Eddy Simulation \\ DNS Direct Numerical Simulation \\ EVM Eddy Viscosity based turbulence Model \\ LES Large Eddy Simulations \\ RANS Reynolds Averaged Navier-Stokes \\ $\mathrm{RC} \quad$ Rotation/Curvature \\ RSM Reynolds Stress Model \\ SA Spalart-Allmaras turbulence model \\ SGS Sub-grid Scale \\ SM Smagorinsky-Lilly SGS model \\ SWBLI Shock Wave Boundary Layer Interaction \\ TBL Turbulent Boundary Layer \\ TKE Turbulent Kinetic Energy \\ WALE Wall-Adapting-Local-Eddy viscosity SGS model
}




\section{Symbols}

\begin{tabular}{|c|c|}
\hline$\alpha$ & Angle of attack \\
\hline$\Delta t$ & Time step size \\
\hline$\Delta x^{+}$, & $\Delta z^{+}$Grid spacing in wall units \\
\hline$\delta^{*}$ & Boundary layer displacement thickness \\
\hline$\kappa$ & Von Karman constant \\
\hline$\nu$ & Kinematic viscosity \\
\hline$\nu_{S G S}$ & Sub-grid scale viscosity \\
\hline$\nu_{t}$ & Turbulent viscosity \\
\hline$\rho_{\infty}$ & Freestream density \\
\hline$\sigma$ & Sigma SGS model \\
\hline$\tau_{w}$ & Wall shear stress \\
\hline$\theta$ & Boundary layer momentum thickness \\
\hline$A^{+}$ & Van Driest constant/variable \\
\hline$C$ & Chord length \\
\hline$C_{f}$ & Skin friction coefficient \\
\hline$C_{P}$ & Pressure coefficient \\
\hline$d$ & Distance to the nearest wall \\
\hline$D_{J L}$ & Modified Van Driest damping function \\
\hline$d S$ & Surface direction \\
\hline$H$ & Shape factor \\
\hline$k$ & Turbulence kinetic energy \\
\hline$K_{S}$ & Acceleration parameter \\
\hline$I$ & Maximum lip thickness \\
\hline
\end{tabular}




$\begin{array}{ll}M & \text { Mach number } \\ M_{\infty} & \text { Freestream Mach number } \\ p & \text { Static pressure } \\ P_{\infty} & \text { Freestream static pressure } \\ Q & \text { Q-criterion } \\ S & \text { Strain rate } \\ T^{*} & \text { Flow through time } \\ T i & \text { Turbulence intensity } \\ u & \text { Streamwise velocity } \\ u_{e} & \text { Boundary layer edge velocity } \\ U_{\infty} & \text { Freestream velocity } \\ v & \text { Wall normal velocity } \\ y^{+} & \text {Non-dimensional wall-normal distance } \\ R e & \text { Reynolds number }\end{array}$





\section{Chapter 1}

\section{Introduction}

\subsection{Motivations}

The rapid growth of civil air travel in recent years is pushing aero-engine manufacturers to develop more fuel efficient engines. This is primarily driven by rising fuel prices, as well an increase in environmental impact.

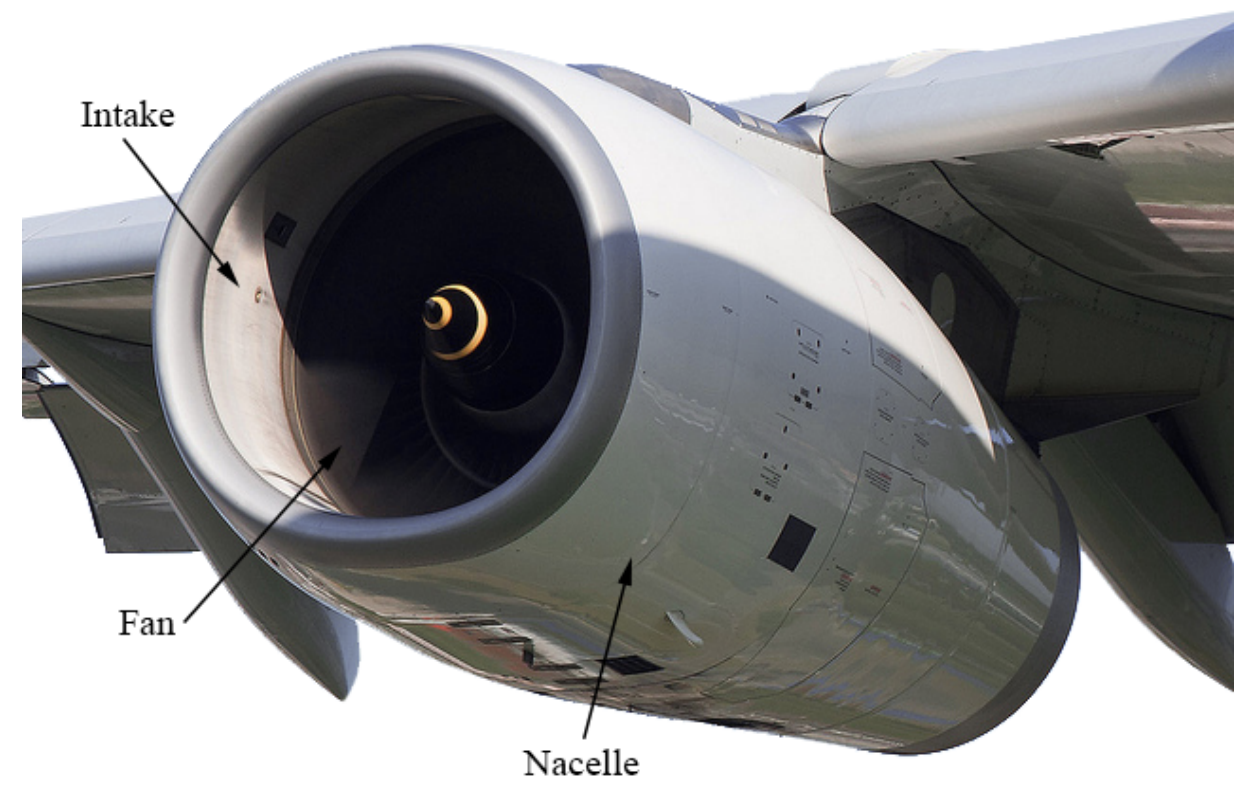

Fig. 1.1 Rolls-Royce Trent-700 at take-off showing the intake section, fan and nacelle

Figure 1.1 shows a typical high-bypass ratio turbofan engine of the type used on most modern airliners. The overall efficiency of these engines is governed by a combination of thermal and propulsive efficiencies. Recent efforts in increasing thermal 
efficiency have plateaued as a results of limits of turbine cooling and material technology. Consequently, efforts have shifted towards improving propulsive efficiencies. In the case of high-bypass ratio turbofan engines, this can be achieved by increasing the fan pressure ratio through larger fan diameters.

However, larger fans bring about many unwelcome side effects. An increase in the fan diameter means an overall increase in the physical dimensions of the engine. This means bigger intakes and nacelles, as shown in figure 1.1. These lead to increased drag (due to increased wetted area) and weight of the engine that could negate propulsive efficiency gains. For under-wing podded-engines, additional adverse effects include: the need for longer (and heavier) landing gear to maintain engine ground clearances; increased interactions between the nacelle and wing, including disruption of nearby wing leading edge high lift devices; and often overlooked logistical issues in transporting these massive engines around the world. Thus, as future engine fan diameters increase, shorter intakes and slimmer nacelles are desired to mitigate the effects associated with directly scaling these components.

Progress in the field is being made. Rolls-Royce's recent announcement of next generation Advance and UltraFan engine designs aim to offer up to $25 \%$ reduction in fuel burn over first generation trent engines, with an entry into service as early as 2025 [17]. In addition to a new core architecture and composite carbon/titanium fan blades, the design of the engine intakes will play a key role in achieving this.

Intakes play a critical role in the performance of the engines. As the initial stage of the engine system, it is their function to provide steady, uniformly distributed air flow to the engine fan face in a variety of operating conditions, all whilst minimising drag and losses [18].

When moving towards shorter intakes and reduced lip thicknesses, new design challenges emerge. A shorter intake length means increased intake fan coupling, with complex intake lip flow interacting more to the engine fan face. If not properly managed, this can lead to increased unsteady flow distortion at the fan that, in addition to fan aerodynamic operability issues, can cause blade fatigue if sustained over a longer period [19]. Shorter intakes also leave less internal area for components such as noise suppression liners, which must move forward into the lip region, increasing the flow complexity here. Sufficient understanding and accurate predictability of intake flow features is therefore crucial when designing engine intakes, to minimise these effects or push them outside of the flight envelope.

Ideally, this close intake-fan coupling calls for the two components to be developed together for optimum performance. However in reality, the long and somewhat 
independent processes of intake and fan design (requiring detailed computational simulation and wind tunnel testing over various design iterations and flight conditions) mean the development time scales are often out of sync. For example at Roll-Royce, the intake geometry is usually fixed to within millimetres before the fan design is even finalised. This design uncoupling is made worse by the fact that most engine manufacturers are not in control of the intake or nacelle geometries themselves, with the airframers defining these. As a result, intake geometries are made less aggressive with regards to optimum performance in order to accommodate uncertainties in fan design $[18,19]$. Thus intake designers do not only need accurate predictive methods, but time efficient ones too, that enable quick turn around times so that intake design can be brought forward and more in phase with the fan design process. Robust methods will also lessen the need for expensive, time consuming wind tunnel testing, supporting this further.

\section{$1.2 \quad$ Intakes at Incidence}

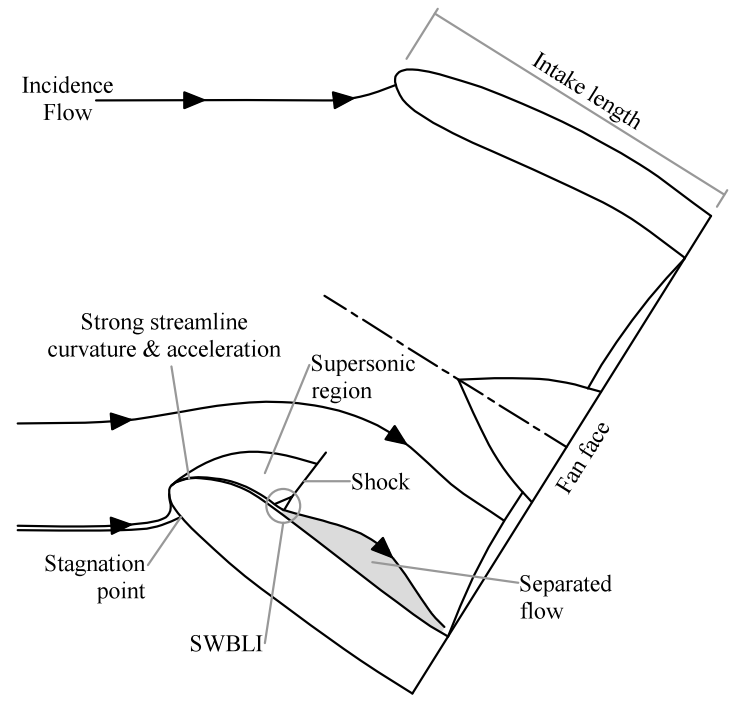

(a) Standard intake

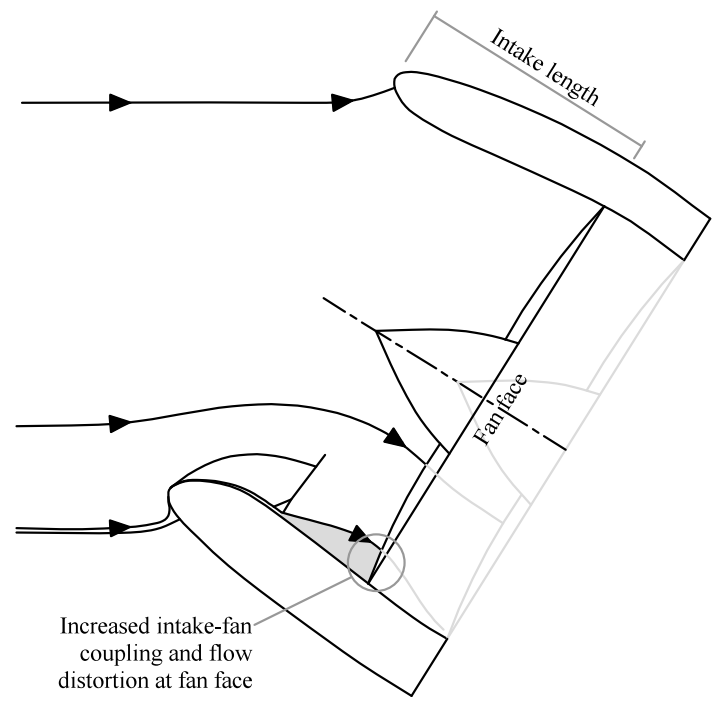

(b) Short intake

Fig. 1.2 Flow features of intakes at incidence

Intakes operating during situations of high incidence flow- e.g. take-off or instantaneous crosswinds/gusts- present a particular challenge, and will be the focus of this work. Under these conditions, the high streamline curvature of the intake lip can accelerate flow to supersonic speeds, creating localised supersonic regions at the intake 
lip, terminating with a near normal shock wave. This shock impacts directly onto a young boundary layer on the inner lip surface, believed to be in a laminar/transitional state. This leads to unfavourable shock-wave boundary layer interactions (SWBLI). If the shock is strong enough, shock-induced boundary layer separation can occur which inevitably leads to strong three-dimensional flow unsteadiness or shock buffet. These key flow features are illustrated in Fig 1.2a. Resultant flow oscillations can propagate far downstream of the interaction, giving rise to severe flow distortion and unsteadiness at the fan face [20]. This can be so severe that they can cause damage to the fan or, if the separation is large enough, can cause fan stall $[6,20]$.

Since the intake shape is fixed, intake geometries must be designed to handle this severely off-design flight condition, compromising performance at cruise to meet certification requirements [18].

With future intakes moving towards shorter, slimmer designs, interactions with this distorted flow will become even more significant. As illustrated in fig. 1.2b, a shorter intake moves the fan much closer to the complex flow features at the lip. This increases the intake fan coupling and the potential distortion experienced by the fan.

Predictive methods must be able to reproduce key flow physics such as accurate reproduction of streamwise acceleration, surface pressure distribution, supersonic region size and shock strength and location, all within reasonable time scales. Furthermore, these should allow accurate assessment of intake characteristics like incidence angles at which flow separation occurs, post separation flow unsteadiness and maximum acceptable distortion levels. This will allow for more refined and efficient intake designs, as well as a more reliable design framework.

\subsection{Computational Fluid Dynamics (CFD)}

With the ever-increasing computational power available today, using computers to accurately simulate fluid dynamics has become a real possibility. Coupled with the use of wind tunnel and full scale engine tests, Computational fluid dynamics (CFD) is extensively used throughout the process of modern aero-engine development.

CFD offers numerous benefits over conventional experimental techniques. Computational models can be created and run over multiple configurations and flow conditions without the time, expense or intrusive measurement methods required to build and test physical components. Furthermore, data can be viewed, investigated, and analysed over and over, with the ability for new information to be derived long after testing. 


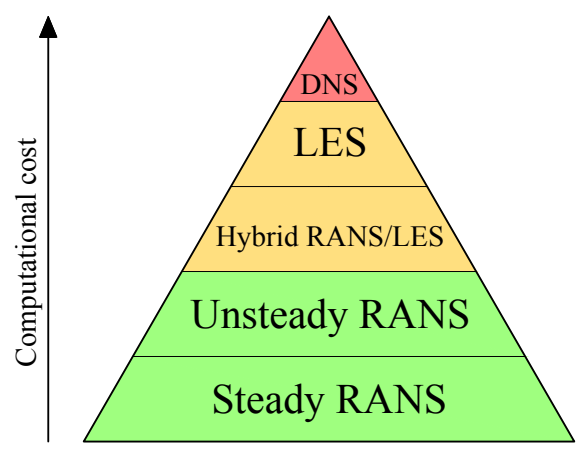

Fig. 1.3 Hierarchy of CFD methods

Despite its wide spread use, CFD methods are still in need of continuous research and development. Although most flows can be fully described by the Navier-Stokes equations of fluid motion, Direct Numerical Simulations (DNS), requiring resolution of all spatial and temporal scales of the turbulence down to the Kolmogorov scales, are still far too computationally expensive for most applications. This has given rise to techniques such as Reynolds-averaged Navier-Stokes (RANS) that model the turbulent motions instead. These provide much more efficient computations for use in commercial environments. However, the variety of models and their suitability (or unsuitability) to certain flow types mean RANS simulations are not truly predictive. They often require sufficient validations before use. Nevertheless, the relative cheapness of RANS means it is still the workhorse CFD tool in industry, and so continuous development and improvement of RANS models is still a necessity. As a result, partnering of computational and experimental efforts still forms an integral part of aero-engine design.

Figure 1.3 presents a hierarchy of the main CFD methods in order of computational cost, with DNS at the top and steady RANS at the bottom. In the middle lie Large Eddy Simulations (LES). LES is an approach where the large turbulent scales that form the bulk of the turbulent motions are explicitly resolved, while only the smallest scales are modelled using a sub-grid scale (SGS) model. While still expensive for wall bounded flows, LES offers far greater fidelity compared to RANS methods since only the smallest scales are modelled, while being orders of magnitude cheaper than DNS.

Further still, it is often desired to combine the advantages of RANS and LES, without bearing the full cost of LES. Hybrid RANS-LES methods provide a framework for this, circumventing the primary cost of LES- the need to resolve fine near wall turbulent structures in boundary layer flows. These instead conduct RANS in the near wall flow and switch to LES away from the wall. This gives an intermediate 
between RANS and LES and is well suited for large separated flows. Hybrid methods such as these look to provide a promising next step for use in industry, bridging the gap between RANS in use today and LES in the future.

Details of the numerics and applications of the aforementioned CFD methods are explored in later chapters.

\subsection{Aims and Research Objectives}

This project aims to study CFD simulations of transitional shock wave boundary layer interactions in engine intakes at incidence. The objective of the work is to improve and assess the modelling of this complex, very interactive and potentially unsteady flow, comparing to new experimental rig measurements. Work is partnered by a novel state of the art experimental effort at the Department of Engineering, University of Cambridge, consisting of a lower intake lip mounted in a transonic wind tunnel ${ }^{1}$. It is believed to be the only such setup, and this project hopes to be the first to carry out and validate CFD of this flow type. The close partnering of experimental and CFD work offers a substantial advantages. As Aeschliman and Oberkampf [25] put it, "careful experiments designed and executed specifically for CFD validations are the recommended source for validation data", which these projects aim to provide.

CFD simulations and development will be undertaken using the Rolls-Royce HYDRA CFD code with the aim of assessing the current capability of the code and CFD methods, as well as testing and implementing additional modules to improve the prediction of intake shock separation flow physics. The work will centre on the following main technical activities:

- Development of improved RANS modelling capability to address deficiencies in current methods for intake flow physics.

- Assess wall-resolved LES and hybrid RANS-LES for intake flow physics prediction.

- Calculations of the experimental intake rig with enhanced models.

The project aims to conclude by providing a hierarchy of predictive strategies, ranging from improved RANS models for current use, to LES methods for future simulations, for an improved predictive framework for future intake design.

\footnotetext{
${ }^{1}$ Experimental work is carried out by fellow $\mathrm{PhD}$ students Tafara Makuni [21, 22] and Andrea Coschignano $[23,24]$ both under the supervision of Professor Holger Babinsky at the Department of Engineering, University of Cambridge
} 


\section{Chapter 2}

\section{Literature Review}

\subsection{Introduction}

This chapter will first review literature on aero-engine intake flows, SWBLIs and related flow phenomena. The search for literature on intake flows at incidence has revealed a lack of work that combines the complex flow features described in the previous section. However, there has been considerable work on CFD modelling of intake flows at cross wind, as well as significant progress on SWBLIs.

As such, a review of literature presented in the following sections will be split into two parts; studies that investigate intake flow, and studies on SWBLIs.

\subsection{Aero-engine Crosswind Flows}

Fig. 2.1 shows the key flow features in crosswind flow. While transonic flow over intakes at less severe incidence angles share some of these characteristics, such as strong lip flow acceleration and large flow separation, they lack some key flow features. These are primarily large, well defined supersonic regions, and strong shock waves and SWBLIs that can significantly alter the flow physics. Nevertheless, if flow velocities are high enough, crosswind flows have been shown to exhibit small scale transonic flows that provide a suitable case for discussion $[1,6]$. These describe the accurate prediction of the shock position and resulting separation as a challenge for RANS models.

Recent work has been done on the prediction of aero-engine intake flows at crosswind by Oriji and Tucker [1, 19], Vadlamani and Tucker [3] and Colin et al. [6]. These have been supported by experimental studies such as those by Hall and Hynes [4, 5] 


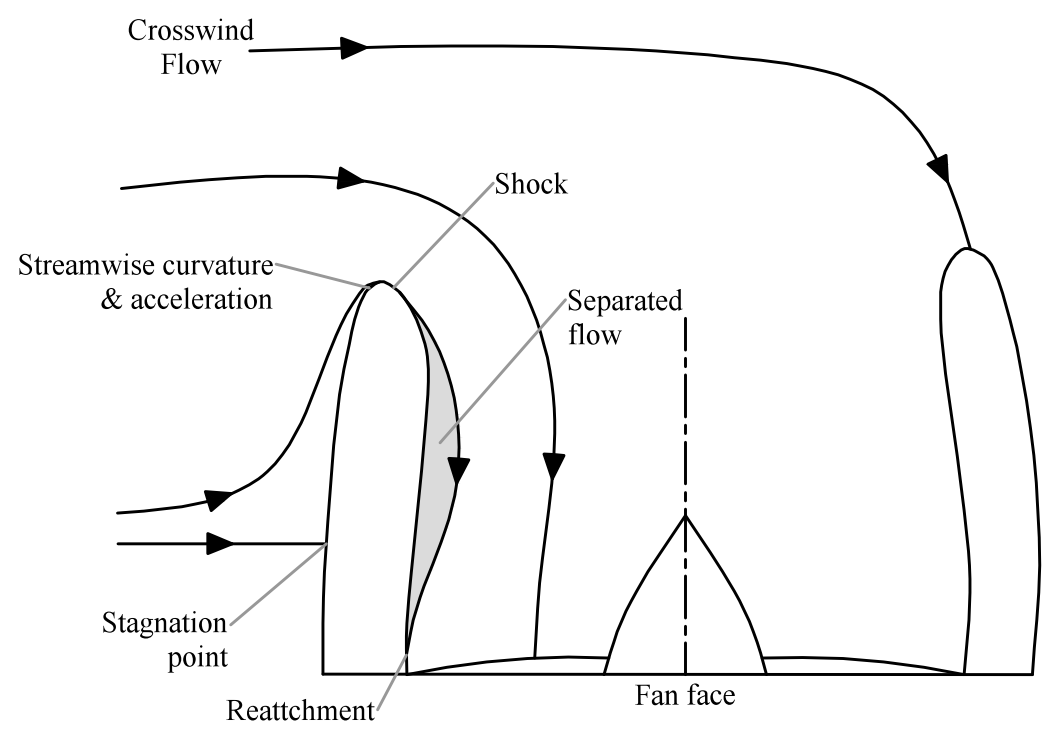

Fig. 2.1 Key flow features of intakes in 90 degree crosswind flow

that aim to provide numerical validation data for such flows, as well as to better understand intake separation mechanisms and downstream flow distortions. Work by Wakelam et al. [18, 26] has also looked at flow control techniques to reduce separation induced flow nonuniformity in intakes that may prove beneficial for future intake design. These studies typically consider intakes operating in pure 90 degree crosswind flows, with a lack of work investigating transonic flow features at less aggressive incidence angles.

Oriji and Tucker [1] identified four key flow zones present in an intake at crosswind that required accurate reproduction to represent the overall flow. These were prediction of accelerating boundary layers, high streamline curvature, boundary layer transition and surface roughness. This coupling of flow features presents a challenge for RANS simulations, whereby turbulence models that are often tailored for certain types of flow must respond correctly to the varying flow regimes.

In particular, highly accelerated flows pose difficulty for RANS models. Flows of this nature can exhibit effects of boundary layer relaminarisation, or reverse transition, whereby severe favourable pressure gradients can revert the boundary layer from a turbulent to a quasi-laminar state. The dimensionless acceleration parameter $K_{S}$ used to discern laminarisation described by Launder and Jones [27] is,

$$
K_{S}=\frac{\nu}{u_{e}^{2}} \frac{d u_{e}}{d s}
$$

where $\nu$ is the kinematic viscosity, $u_{e}$ is the velocity at the boundary layer edge 
and $s$ is the flow streamline direction. Loyd et al. [28] and Launder and Stinchombe [29], suggested the acceptable threshold for which laminarisation occurs is between $2.5 \times 10^{-6}$ and $3.5 \times 10^{-6}$ [19]. Oriji and Tucker [1] showed that $K_{S}$ can peak to values up to $4 \times 10^{-6}$ for intakes in crosswind [1] which is more than sufficient to laminarise the boundary layer in this zone. Subsequent interactions of this laminar flow with a shock can induce premature boundary layer separation. In addition to a decay in turbulence, laminarisation is accompanied by flow features such as an increase in the viscous sub-layer and shape factor $H$ and a reduction in boundary layer thickness [19]. These can significantly influence surface pressure distributions and boundary layer profiles. It is therefore crucial to understand and accurately predict reverse transition when designing engine intakes.

Reynolds-Averaged Navier Stokes (RANS) turbulence models often perform well for the prediction of attached boundary layer flows, however most have been shown to be insensitive to relaminarisation and need modification [30]. The main difficulty faced by RANS models in regions of laminarisation is the need for the model to stop net production of turbulence in the laminarisation zone, and resume production afterwards. Standard models remain turbulent in regions where experiments show decay, as examined by Rumsey and Spalart [19, 31]. Karimisani et al. [30] also showed in a recent study that most RANS models fail to capture the effects of laminarisation, with only the Launder Sharma, Explicit algebraic stress and Cubic models able to predict laminarisation behaviour correctly in their standard forms.

In addition to acceleration, strong streamline curvature is also an important consideration for intake flows. The presence of a large favourable pressure gradient over the convex lip can exhibit similar behaviour to accelerating boundary layers (i.e. laminarisation), and the combination of these together can make a more rapid and complete reversion to laminar flow [19]. It is well-known that many eddy-viscosity-based turbulence models (EVMs) fail to capture curvature well [19, 32], as opposed to the Reynolds stress model (RSM), whose sensitivity to curvature is considered a fundamental advantage [32, 33]. However, the increased complexity of RSM with up to seven governing equations, high computational cost, and notorious difficulties in simulation convergence make it unappealing [32]. As a result, sensitising simpler EVM models to curvature is desired. A number of corrections to this effect have been proposed [1, 32, 33], the most notable being that by Spalart and Shur [33]. They proposed a rotation/curvature $(\mathrm{RC})$ correction that can be applied to one and two equation turbulence models, which introduces an additional transport equation to account for the effects of RC. This has been shown to be much more accurate than the standard 
models for a wide range of rotating and curved flows [32, 34]. Simpler alternatives such as that proposed by Zhang and Yang [32] based on the Richardson (Ri) number, negate the complexity of implementation and increased computational cost imposed by the RC function of Spalart and Shur [33] and have been shown to give competitive if not superior results.

It is also well known that RANS is fundamentally unable to predict separated flows regions well, such as separation bubble size, where modelled turbulence production in separated shear layers is often insufficient to correctly reattach the flow [1]. This is due to the RANS formulation's inability to account for the three dimensional unsteady turbulent motions present here. For intake simulations, this means RANS tends to over-predict the separation size. More advanced eddy resolving methods such as LES are able to resolve turbulence scales directly, however are far too computationally expensive in an industrial environment.

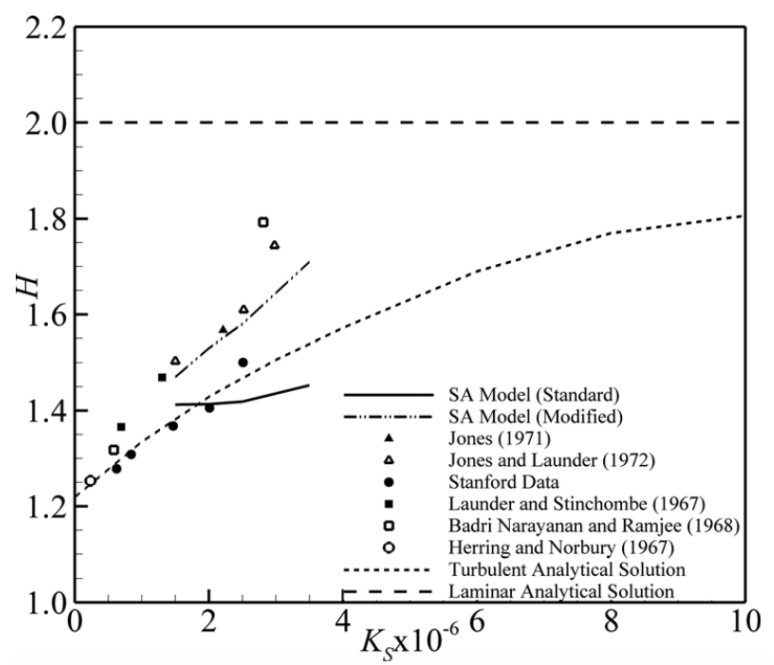

Fig. 2.2 Variation of shape factor with acceleration parameter in accelerating flow in a nozzle from Oriji and Tucker [1]

Oriji and Tucker [1] presented corrections to the one equation Spalart-Allmaras turbulence model in a modular form, applying modifications for laminarisation, curvature, transition and roughness. Their laminarisation correction involved damping of the turbulence viscosity in regions of high $K_{S}$. Fig. 2.2 shows the variation of shape factor $(H)$ with $K_{S}$ from [1] for the standard and modified SA models, together with some validation data. $H$ is used to characterise the nature of boundary layer flow, 
defined,

$$
\begin{gathered}
H=\frac{\delta^{*}}{\theta} \\
\text { where } \delta^{*}=\int_{0}^{\infty}\left(1-\frac{\rho(y) u(y)}{\rho_{0} u_{0}}\right) d y, \quad \theta=\int_{0}^{\infty} \frac{\rho(y) u(y)}{\rho_{0} u_{0}}\left(1-\frac{u(y)}{u_{0}}\right) d y
\end{gathered}
$$

$\delta^{*}$ and $\theta$ are the boundary layer displacement and momentum thicknesses respectively for a compressible flow. $\rho_{0}$ and $u_{0}$ are the density and velocity outside the boundary layer respectively, and $y$ is the wall normal coordinate. $\delta^{*}$ and $\theta$ represent the distance a viscous wall would have to be displaced normal to its surface to give the same flow rate or total momentum in an inviscid solution respectively. $H$ is usually in the range 2.0-2.6 for laminar flow, and 1.2-1.4 for turbulent flow [1]. As can be seen from fig. 2.2, the standard SA model maintains a relatively constant $H$ with increasing $K_{S}$, indicating insensitivity to laminarisation, whereas the modified model correctly shows reversion to laminar flow [1]. The modified model was applied for the prediction of crosswind flow at $M=0.55$ around the lip of a 90 degree sector of an intake as shown in fig. 2.3.

Fig. 2.3b shows the predicted size of the separation bubble on the inner intake lip during pure crosswind flow using the standard and modified SA models, with the modified model matching much closer to experiment. The standard SA model predicts a separation bubble much larger than is experimentally measured. This results in the surface isentropic Mach number $\left(M_{i s}\right)$ on the inner intake lip being incorrectly predicted as shown in figure $2.3 \mathrm{~d}$. The standard model shows a flat $M_{i s}$ due to the large separation, while the modified model is able to capture the $M_{i s}$ that agrees well with experimental measurements. The standard model's overprediction of the separation also causes a significantly large total pressure loss at the fan face as shown in figure $2.3 \mathrm{c}$ which would make it unreliable for intake-fan predictions.

The significant difference in the flow field between the standard and corrected SA models, namely the separation bubble size, highlights the importance of correctly modelling the aforementioned flow features. 

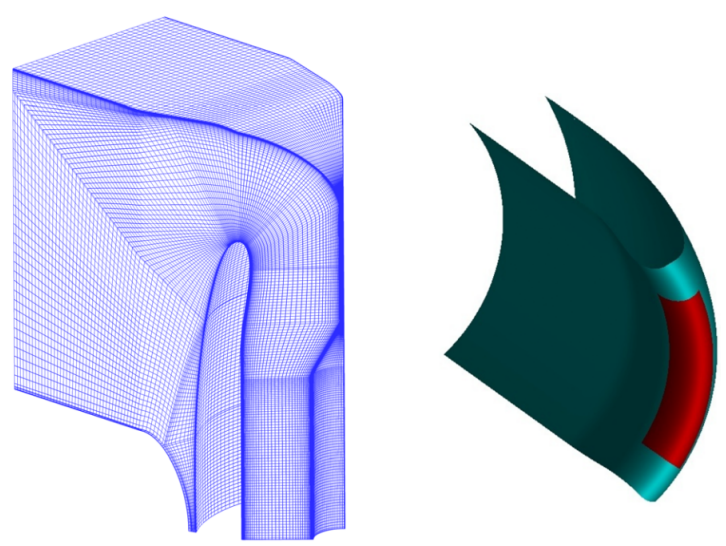

(a) Intake lip geometry and mesh

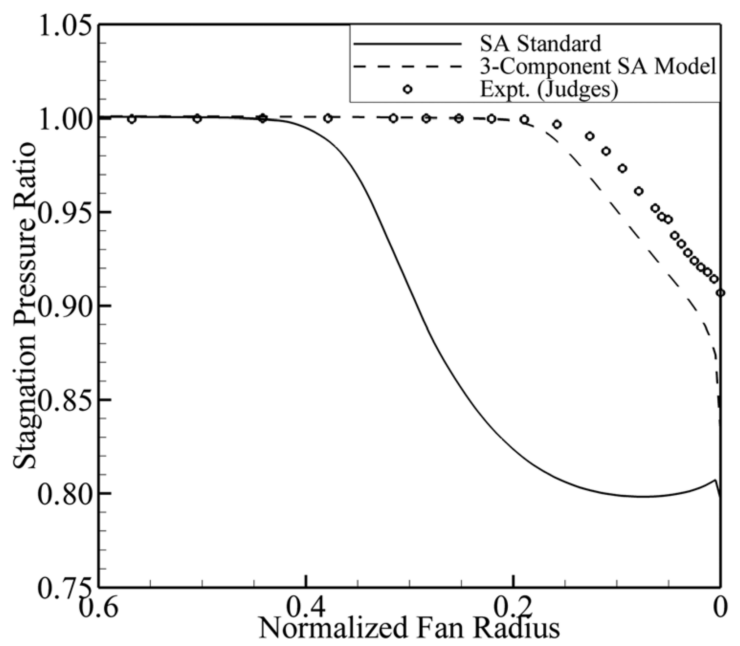

(c) Stagnation pressure ratio at the virtual fan face

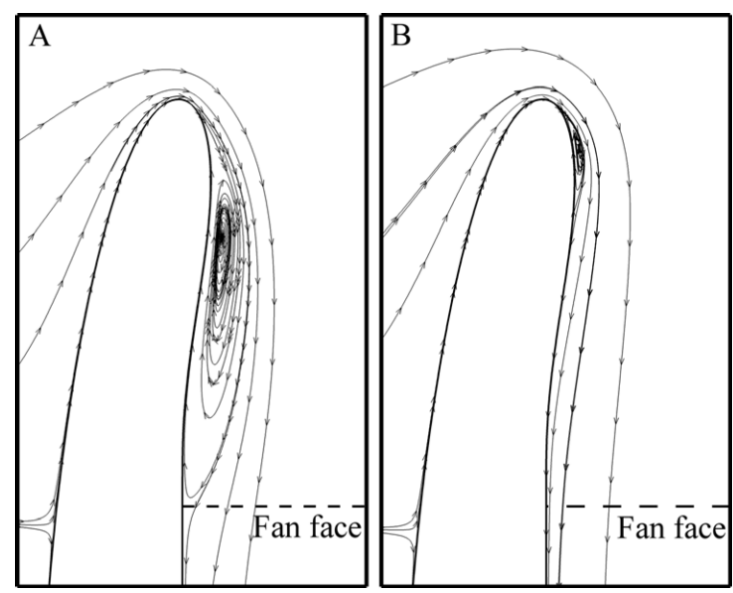

(b) Predicted intake separation with the standard model(A) and modified model (B)

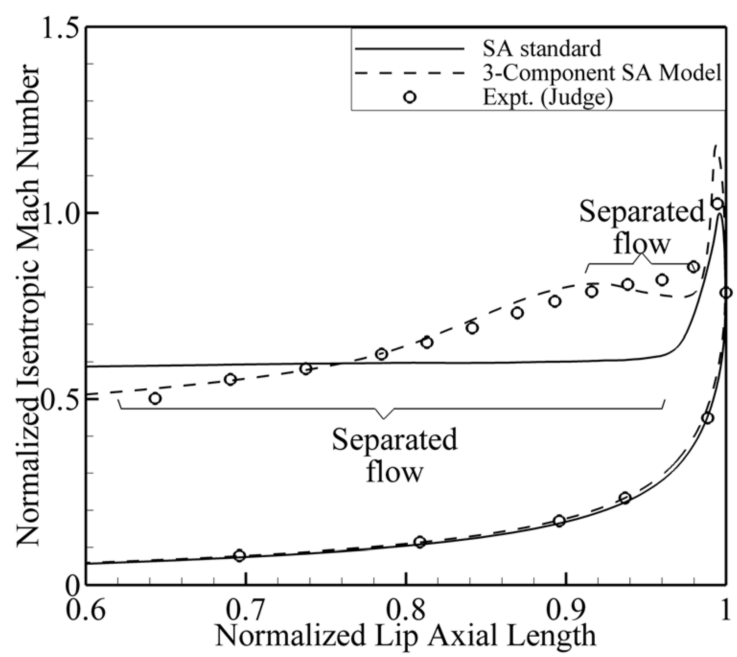

(d) Lip isentropic Mach number

Fig. 2.3 Intake rig case details and results by Oriji and Tucker [1] for pure crosswind flow, with the modified (3-component) SA model matching much closer to experiment

Oriji and Tucker [2] also carried out hybrid RANS-LES of this crosswind flow configuration, using their enhanced SA model in a RANS layer around the intake lip surface, and implicit LES away from the wall. Hybrid RANS-LES methods aim to circumvent the most computationally demanding task for LES - the need to resolve extremely fine flow structures in boundary layers. The relatively simple subgrid scale (SGS) models used for LES are unable to accurately account for these, and so they must be resolved fully [35]. This means that for LES, computational grids in the boundary layer must be sufficiently fine to resolve these directly. Table 2.1 shows 
the recommended grid resolutions in wall units from Tucker [16] for different eddy resolving methods.

\begin{tabular}{|c|c|c|}
\hline Method & $\Delta x^{+} / \Delta y^{+} / \Delta z^{+}$ & Number $y^{+}<10$ points \\
\hline DNS & $10-15 / 1 / 5$ & $3-5$ \\
\hline Wall resolved LES & $50-130 / 1 / 15-30$ & $3-5$ \\
\hline Hybrid RANS-LES & $100-600 / 1 / 100-300$ & $2-5$ \\
\hline RANS & $1000 / 1$ & $2-5$ \\
\hline
\end{tabular}

Table 2.1 Grid requirements for different eddy resolving methods from Tucker [16]. $\Delta x^{+}, \Delta y^{+}$and $\Delta z^{+}$are the recommended grid spacings in the streamwise, wall normal and cross stream directions respectively.

Non-dimensional wall units are defined,

$$
y^{+}=\frac{y}{\nu} \sqrt{\frac{\tau_{w}}{\rho}}
$$

where $y$ is the physical distance to the nearest wall and $\tau_{w}$ is the wall shear stress $\left(\tau_{w}=\right.$ $\left.\mu(\partial u / \partial y)_{y=0}\right)$. As the Reynolds number (Re) increases, the physical grid dimensions needed to meet these grid requirements decreases. In addition to this, timestep size must also accordingly reduce to maintain simulation stability, thus needing more steps for a given simulation time [35]. This can lead to extreme computational demands that make LES infeasible for high Re flows.

Hybrid RANS-LES methods aim to limit this computational expense of LES by instead modelling the fine turbulent structures within the boundary layer with a RANS layer, and blending this near wall solution with LES away from the wall. This significantly relaxes the grid requirements as shown in table 2.1 and is particularly suited to large separated flows. Many hybrid RANS-LES techniques have been proposed over the years. One of the most widely used of these are Detatched-Eddy Simulations (DES) proposed by Spalart et. al [36] that use a modified wall distance term $\tilde{d}$ based on the mesh size $\Delta$ and true wall distance $d$, to move between SA RANS and LES regions. $\tilde{d}$ is given by,

$$
\tilde{d}=\min \left(d, C_{D E S} \Delta\right)
$$

where $C_{D E S}$ is a constant. This gives an adaptive turbulence model whereby the RANS-LES interface can vary depending on the mesh. However, effort must be made to carefully design the mesh for DES to ensure this interface sufficiently encapsulates the whole boundary layer region. Oriji and Tucker [2] avoid this mesh dependency 
by using a zonal approach where the RANS layer is used for only the inner part of the boundary layer and is fixed at $y^{+}<60$. A similar approach was also used by Davidson and Peng [37]. This method aims to only model the very near wall streaks (that arguably require high resolution). LES is used for $y^{+}>60$.

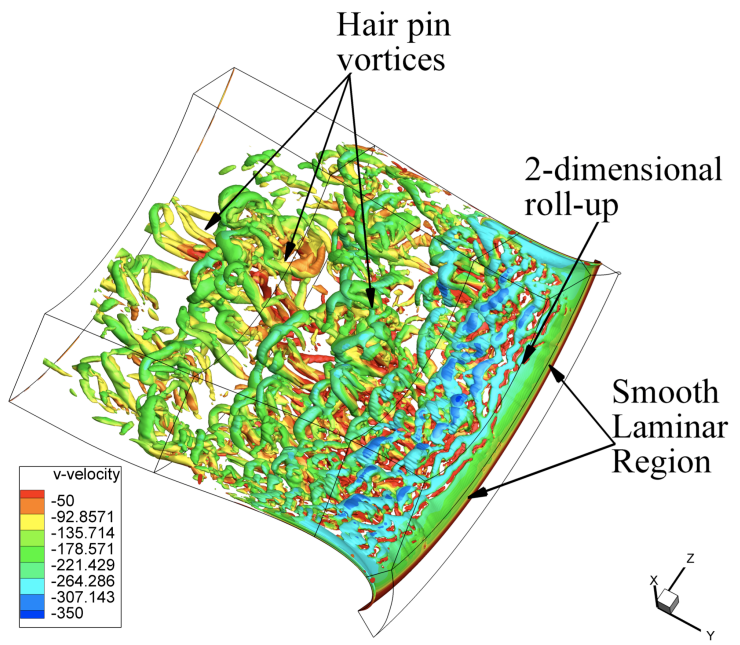

(a) Iso-surface of Q around the lip using hybrid RANS-LES

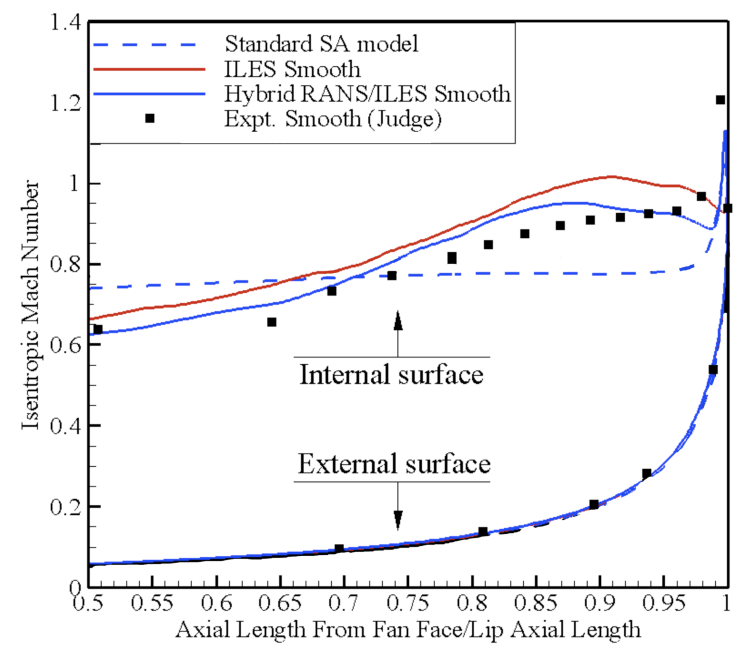

(b) Lip isentropic Mach number

Fig. 2.4 Hybrid RANS-LES results of crosswind flow over an intake lip by Oriji and Tucker [2]

Figure 2.4a shows iso-surfaces of Q-criterion displaying vortical flow structures around the intake lip under crosswind using hybrid RANS-LES by Oriji and Tucker [2]. $\mathrm{Q}$ is defined,

$$
Q=\frac{1}{2}\left(\Omega^{2}-S^{2}\right)
$$

where $\Omega=\sqrt{2 \Omega_{i j} \Omega_{i j}}$ and $S=\sqrt{2 S_{i j} S_{i j}}$ are the vorticity and strain magnitudes respectively, and is used to identify vortical structures in the flow. The figure shows the smooth laminar region over the intake lip as the flow highly accelerates here, before transitioning and breaking down to large scale turbulence. The exit Mach number used for this case was $M=0.58$ with a modest grid of 33 million nodes and wall grid spacing of $\Delta y^{+}=1, \Delta x^{+}=360$ and $\Delta z^{+}=250$. It was found that conducting pure LES on this grid over-predicted the isentropic Mach number $M_{i s}$ on the lip and failed to capture peak $M_{i s}$ as shown in figure 2.4b. This was expected as the grid was not fine enough for LES to resolve the small scale near-wall turbulence, causing premature flow separation. Adding the RANS layer (albeit using an enhanced RANS model) performed significantly better here, clearly showing the benefits for this type of flow.

Athough LES is costly, the superior physical insights that can be obtained provide 
motivations for LES in an academic environment. These can feed back into improving lower-order methods. Vadlamani and Tucker [3] conducted wall resolved LES of intake crosswind flow at reduced Re to explore the effects of Re and flow separation. Their setup followed the experimental work of Wakelam et al. [18] who investigated separation control over an aeroengine intake lip operating in crosswind. To keep computational cost low, a spanwise extrusion of the lip profile by $20 \%$ lip length was used as the computational domain, as opposed to the three-dimensional lip sector used in experiments. Inviscid walls were also used at the wind tunnel walls to minimise grid requirements further. Nevertheless, around 55 million grid nodes were used for simulations at two different Re: $1 \times 10^{5}$ and $4 \times 10^{5}$.

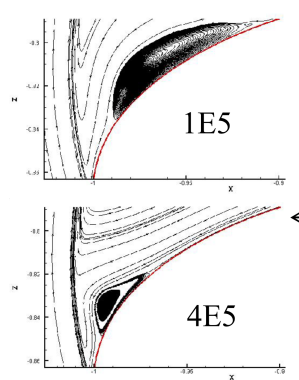

Secondary separation

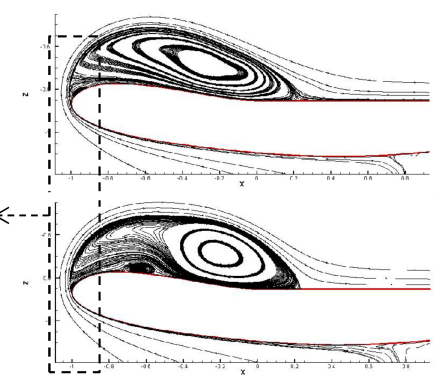

Primary separation

(a) Flow separation around the lip at two Re

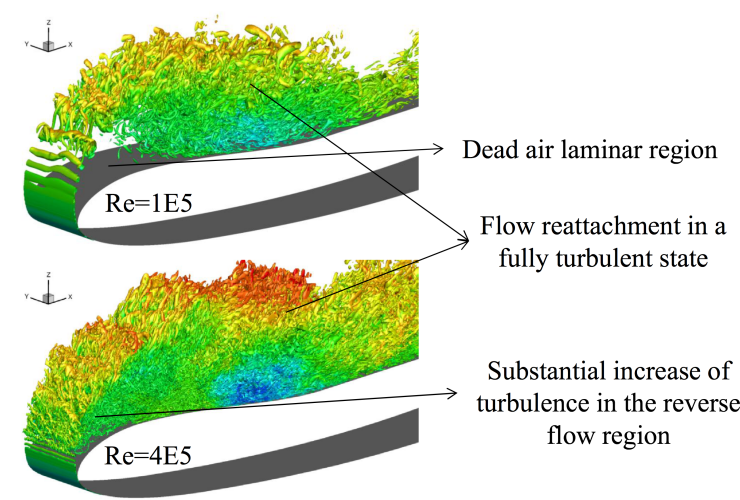

(b) Iso-surface of Q around the lip at two Re

Fig. 2.5 Flow around an intake under crosswind using LES from Vadlamani and Tucker [3]

Results showed that Re had minimum influence on the size of the primary separation over the intake lip as the reattachment process is entirely driven due to turbulence in the separation. However, a secondary separation bubble is typically formed due to the flow reversal and the length of this decreased with increased Re. This is shown in figure 2.5a. It was further observed that the turbulence in the reversed flow is much higher with increasing Re. This can be seen in figure $2.5 \mathrm{~b}$ where due to an increase in the inertial force of the flow with increasing Reynolds number, the energy cascades down to much more finer scales. It is this increase in turbulence with higher Re that results in the suppression of the secondary separation bubble.

In addition to predicting correct size and location of flow separations, distortion levels at the fan face are also desired to assess maximum acceptable limits and intake suitability. Hall and Hynes [4, 5], Motycka [38] and Longley and Greitzer [39] have studied inlet distortion effects experimentally, in addition to CFD of inlet distortions 
by Cao et al. [40], Colin et al. [6] and Peters et al. [41]. These have been studied in a variety of flow conditions.

Cao et al. [40] carried out an extensive numerical study on the interaction between the downstream fan and the flow separating over an intake under high incidence using both steady and unsteady RANS. A low order fan model was used to represent the fan for steady simulations while a full 3D geometry was used for unsteady RANS. Varying the ratio of the intake length to diameter (L/D) was investigated. Results showed that at low $\mathrm{L} / \mathrm{D}$, an increase in the separation-free angle of incidence was achieved, which decreased with increasing L/D. At high L/D, the fan was effective in suppressing the post-separation distortion rather than entirely eliminating the separation. Simulations revealed that the fan accelerates the flow upstream of the fan face, thus decreasing the distortion level in the immediate vicinity, although this effect decayed rapidly with increasing upstream distance from the fan-face. Peters et al. [41] also studied inlet and nacelle design for low fan pressure ratio (FPR) propulsors with large fan diameters and short nacelles, where the fan and nacelle are more closely coupled. A low order body-force-based model was also used for the fan rotor and stator blade rows. They identified the interaction of the rotor with a region of high streamwise Mach number at the fan face as the key mechanism limiting the design of short inlets. For a candidate short-inlet design with $\mathrm{L} / \mathrm{D}=0.19$, they found the streamwise Mach number at the fan face to increase by up to 0.16 at cruise and by up to 0.36 at off-design conditions relative to a long inlet propulsor. This caused penalties in fan efficiency due to the rotor locally operating close to choke. For inlets with $\mathrm{L} / \mathrm{D}<0.25$, the benefit from reduced nacelle drag was offset by the reduction in fan efficiency. These studies demonstrate the importance of carefully defining intake design paramteters, in addition to those such as Andrew et al. [42] that describe the redesign of an inlet/fan cowl for increased angle of attack requirements of transport aircraft.

Unsteady flow conditions can be potentially troublesome for intake performance, yet are often neglected in the steady tests and computation typically carried out [4]. Atmospheric flow can be naturally unsteady or gusting and so consideration of this real world situation is needed, although very little previous work exists [4]. Hall and Hynes [4] presented the first comprehensive experimental study on interactions of the flow in an operating engine model with unsteady atmospheric turbulence. Fig. 2.6 shows a image of flow structures around the intake. The high straining of the flow is immediately visible, with the rapid flow contraction showing stretching of the turbulence flow field and turbulent flow structures. 


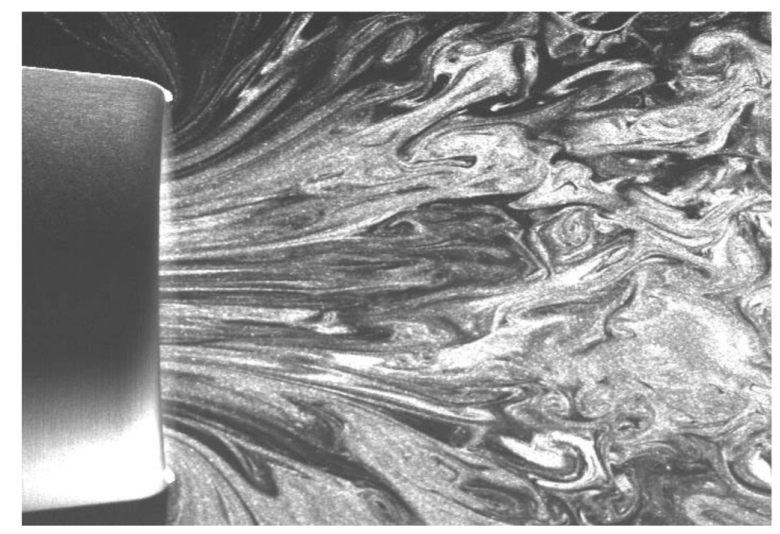

Fig. 2.6 Turbulent flow structures around an intake model by Hall and Hynes [4]

Results also showed significant hysteresis in the intake separation when considering unsteady flow. Accurate measurements of hysteresis made in a further study by Hall and Hynes [5], showed that once separation had occurred, the size of the distortion increased with increasing the wind angle, yet as the angle was reduced, reattachment occurred at a much lower angle than required for separation. This should be taken into account when testing intakes under crosswind conditions. The presence of the fan was found to have negligible effects on the onset of separation, but had a strong influence on reattachment once separation had occurred. The fan pulled flow into the separated wake and reduced its size, promoting reattachment at greater flow angles. This motivates the need for coupled fan-intake predictive methods, such as fan representing bodyforce models, that are more representative of the complete flow. Fig. 2.7 shows contours of loss coefficient at the fan face with the intake flow attached and separated, showing the severe distortion at that fan face that separation can produce.

Colin et al. [6] tested nine RANS turbulence models frequently employed for aeronautical flows to assess their predictive accuracy in computing complex threedimensional separations. The models considered were the algebraic model of Baldwin and Lomax, both basic and rotation-curvature corrected versions of the SA oneequation models, $k-l$ and three different $k-\omega$ models, the nonlinear eddy-viscosity model of Wallin and Johansson, and the differential Reynolds stress model (RSM) of Speziale, Sarkar and Gatski. Models were tested on a three-dimensional intake geometry, shown with a cross sectional Mach number distribution in fig. 2.8.

It was found that none of the models were completely satisfactory at agreeing with with experimental data. Each model possessed its own unique strengths and weaknesses. The SA, $k-\omega \mathrm{SST}$ and RSM models for example were more accurate in the separated flow region, but failed to predict boundary layer reattachment correctly. 


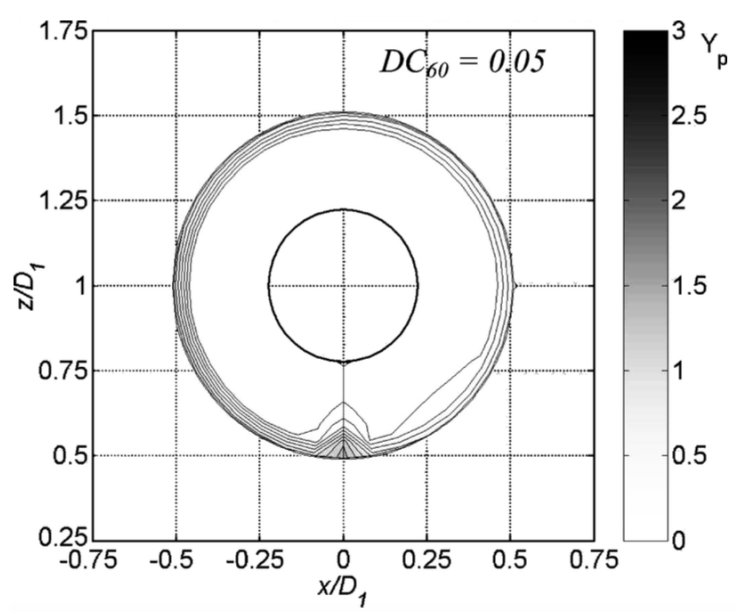

(a) Attached intake flow

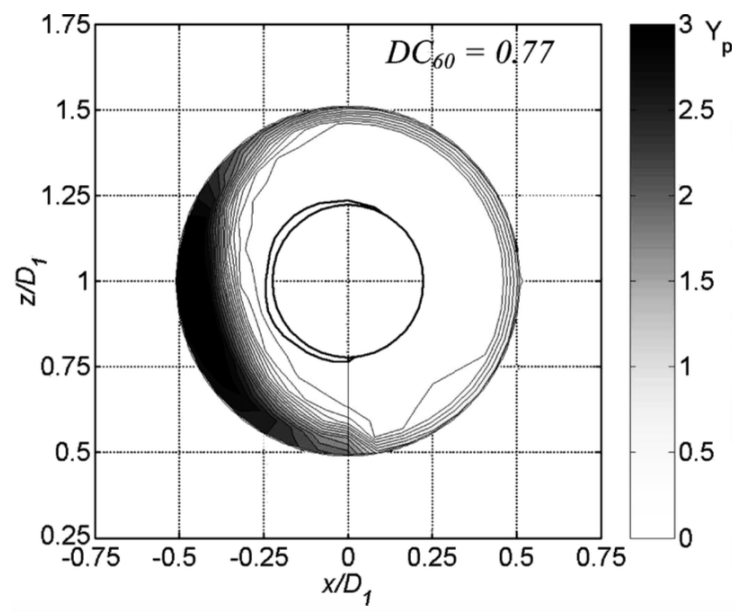

(b) Separated intake flow

Fig. 2.7 Contours of loss coefficient at the fan face with the intake flow attached and separated from Hall and Hynes [5]

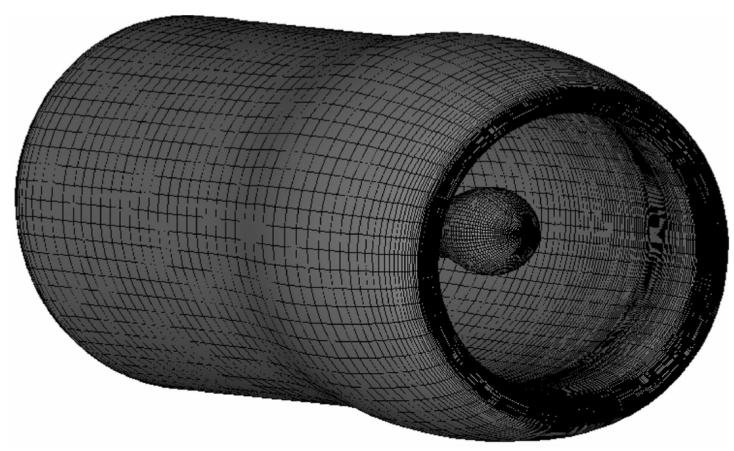

(a) Intake geometry and mesh

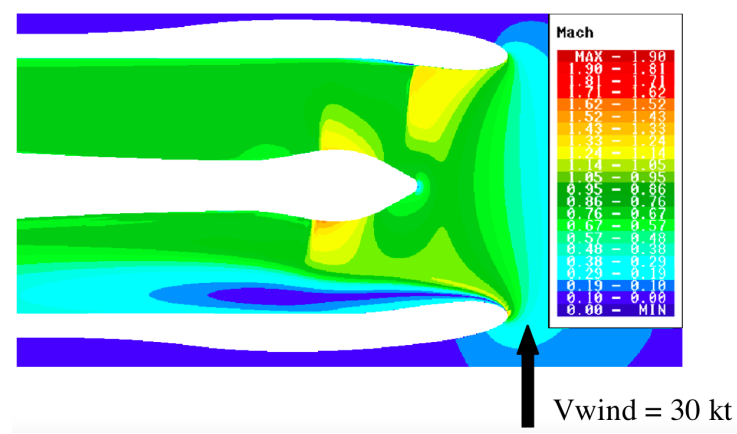

(b) Mach number distribution

Fig. 2.8 3D Intake geometry and crosswind flow separation over an intake lip by Colin et al. [6]

This displays one of the primary drawbacks with RANS models, with no 'one model fits all' approach possible. Models need continuous assessment for specifics flows types to determine best practises. While corrections to model deficiencies can be introduced [1], inherent weaknesses in the RANS formulation will always be a fundamental drawback. For example, the eddy viscosity approach that forms the basis of most RANS models assumes an isotropic character for the turbulent motions, making it difficult to capture the three-dimensional time dependant anisotropic motions of highly separated flow [6]. Nevertheless, the speed, simplicity and practical usability make RANS methods the "work horses" in the aerospace industry, and so RANS recommendations must continue for the foreseeable future [6]. 


\subsection{Shock Wave Boundary Layer Interactions (SWB- LIs)}

SWBLIs can be found in almost every transonic and supersonic flow. In almost all cases, these interactions lead to extremely undesirable effects that include increased drag, flow separation, shock unsteadiness and high wall heating [8]. Fig. 2.9 shows an example of this with shock induced flow separation over a transonic aerofoil. In unsteady cases in particular, strong flow fluctuations are prejudicial to mechanical behaviour and in some cases can cause fatigue or structural damage [8]. As a result, SWBLIs have been the subject of several experimental and numerical studies, and must be an important design consideration when dealing with such flows.

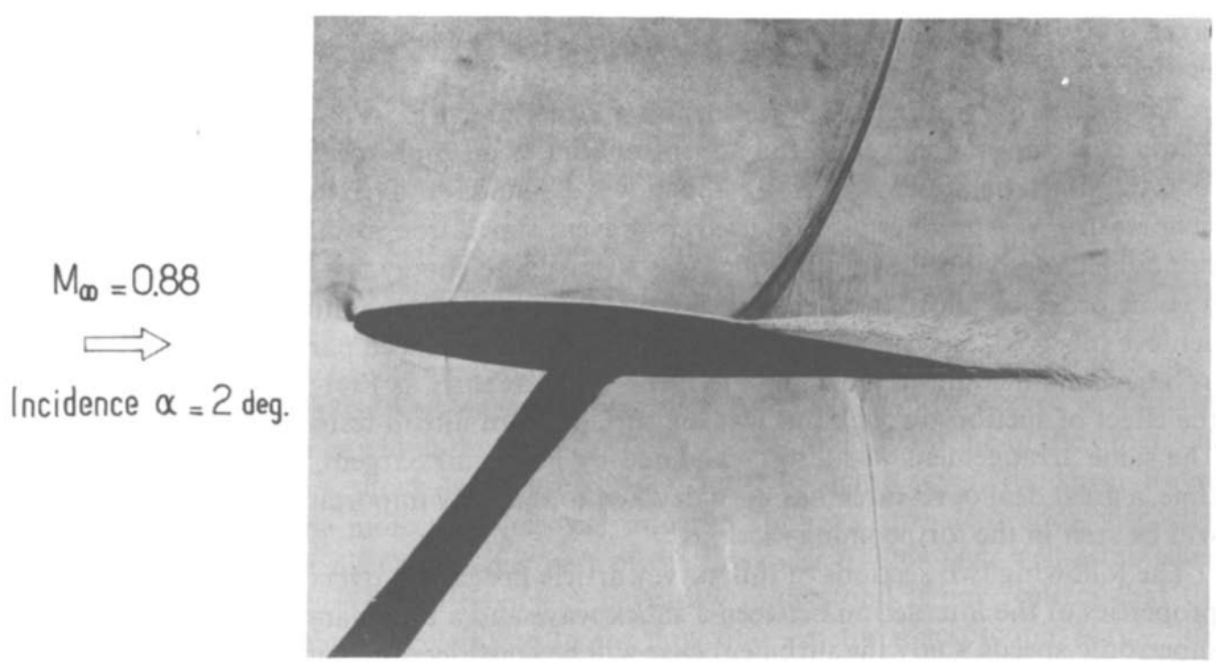

Fig. 2.9 Shock induced flow separation over a transonic aerofoil from Delery [7]

SWBLIs can be characterised into a variety of distinct types. These arise from the various natures of the component boundary layers and shocks waves depending on the flow regime. Their coupling in SWBLIs also mean predictive methods must accurately model these individually, in order to stand a chance at modelling the overall interaction.

Shock waves in transonic and supersonic flow bring about unique flow properties. Flow across a shock wave undergoes a discontinuous decrease in velocity, an abrupt increase in pressure, a rise in flow temperature and a decrease in stagnation pressure [20]. The sudden increase in pressure in particular has several major consequences on boundary layer flow. Boundary layers that are subjected to a shock experience a strong adverse pressure gradient that thickens it and may separate it depending on 
the nature of the interaction. Moreover, the nature of the boundary layer itself is critical to the outcome of the interaction, and can have a strong influence on this [43]. Laminar boundary layers for example are less resilient to adverse pressure gradients, and may separate prematurely. Perturbations caused by the shock could also cause the boundary layer to transition to a turbulent state much earlier than it naturally would. These can have a significant impact on the overall flow.

Schematics of three of the most typically found SWBLIs are shown in fig. 2.10. Note the $\lambda$ shaped foot at the base of the shocks, where the compression waves spread out in the vicinity of the viscous boundary layer. SWBLIs found in aero-engine intakes at incidence are of the transonic normal shock type, and thus will form the focus of this work. Normal shock waves are produced in transonic flows by a back pressure that forces the flow to return to subsonic [20]. The near normal orientation of the shock with respect to the oncoming flow means that the flow decelerates through the shock without significant deflection of the velocity vector; a distinctive feature of normal shocks [20]. However, the flow downstream of a normal shock is subsonic and this can lead to specific problems. Downstream disturbances can influence the flow upstream, interacting with the shock, and can lead to large scale unsteadiness and shock buffet [20]. This is contrary to a pure supersonic flow, where the downstream flow is also supersonic, 'protecting' the shock from upstream propagation of disturbances [20]. As a result, transonic normal shock waves present a particular challenge for predictions. The flow in all regions of the domain becomes closely coupled with the shock dynamics, and accurate predictions of boundary layers both upstream and downstream are required to represent the flow well. SWBLIs can be further categorised into strong and weak interactions, where the interactions occur with and without boundary layer separation respectively.

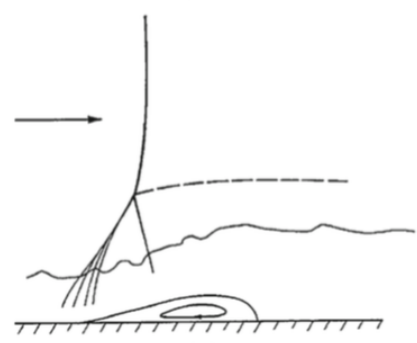

(a)

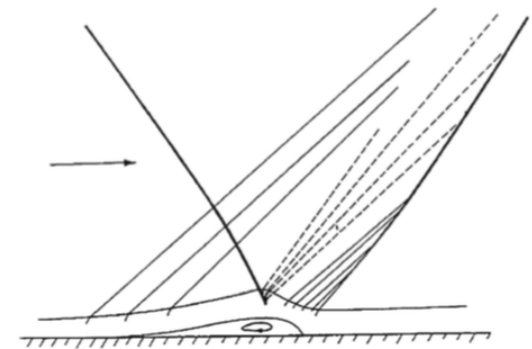

(b)

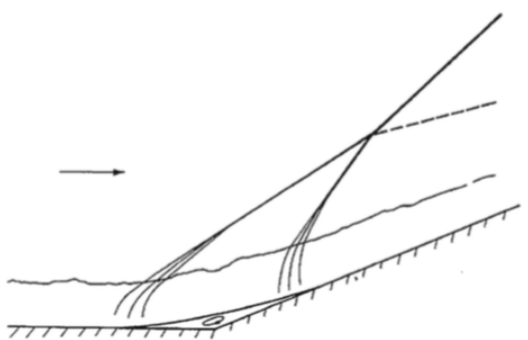

(c)

Fig. 2.10 Typical SWBLIs from [8]. (a) Transonic normal shock (b) Oblique shock (c) supersonic flow over a compression ramp

Numerous review papers can be found on SWBLIs such as those by Delery [7], 
Delery and Marvin [9] and Dolling [43], that give a detailed account of SWBLIs and a rich overview on the works that have been done. Reading material such as Babinsky and Harvey [20] also provide a comprehensive explanation of this phenomenon in all flow regimes where it occurs.

Some examples of typical SWBLIs experimental test cases are shown in fig. 2.11 from Delery [9]. These include the experimental work such as that of Bachalo and Johnson [44] (shown in fig. 2.11g) which have been designed and carried out specifically to provide validations for CFD codes. The axisymmetric hump model for this case for example allows a pure 2D computational approach, as well as measurements of turbulence intensity and Reynolds shear stress profiles for turbulence model validation.

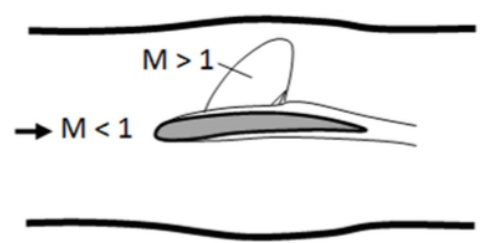

(a) Complete aerofoil

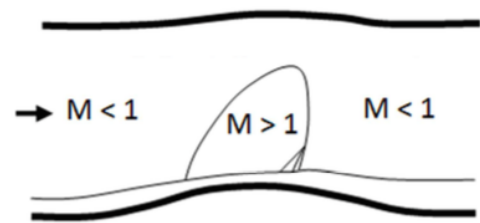

(c) Bump on wind tunnel floor

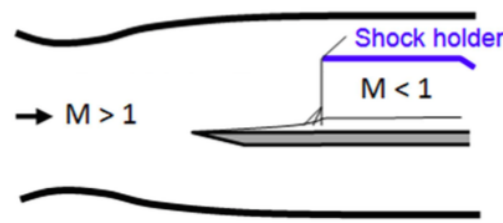

(e) Flat plate with shock generator

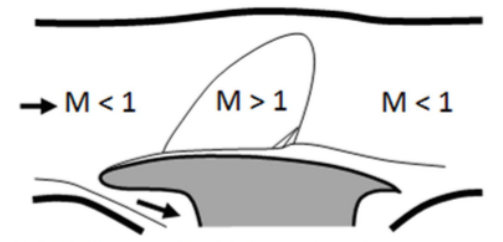

(b) Half aerofoil with boundary-layer removal

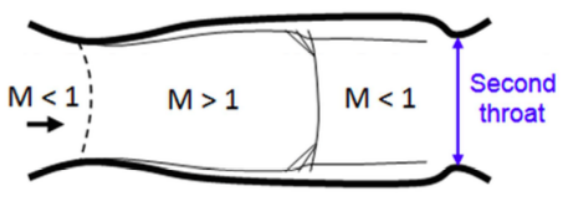

(d) Symmetrical supersonic nozzle

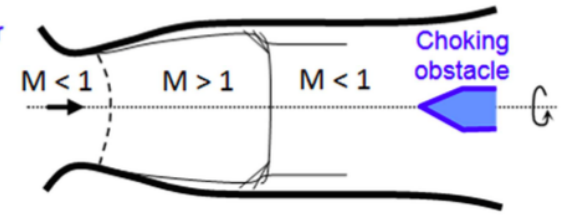

(f) Axisymmetric Supersonic nozzle

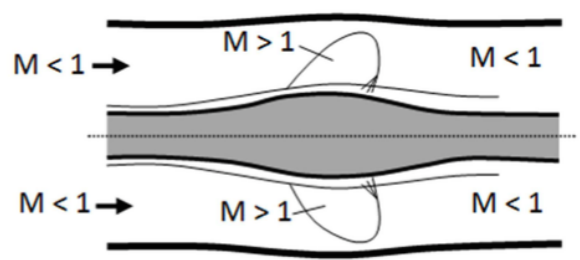

(g) Toroidal bump in axisymmetric flow

Fig. 2.11 Typical experimental test cases for the study of SWBLIs from [9] 


\subsubsection{CFD of SWBLIs}

Ackeret et al. [45] and Liepmann [46] pioneered research on SWBLIs with the first experimental studies on the subject in the 1940s. Since then, the multitude of experimental and numericals studies on SWBLIs has lead to remarkable progress in the computations of SWBLIS, and numerous review papers that aim to summarise the CFD capabilities of current methods. These include work by Zheltovodov [47], Knight and Degrez [48], Knight et al. [49] and Hadjadj and Dussauge [8].

Knight et al. [49] reviewed the advances in CFD predictions of SWBLIs in 2009. The review studied simulation methods including DNS, LES and RANS, and computed results were compared with experimental data to assess the overall capabilities of current CFD methods. Five separate configurations were considered, namely, a 2D compression corner, 2D shock impingement, 2D expansion-compression corner, 3D single fin and 3D double fin. For DNS and LES, Knight et al. [49] note that although computational resources have increased greatly in recent times, the computational cost of these methods means that simulations at experimental Re, or in full 3D, are still not possible. Nevertheless, significant progress has been made at Re close to experiments, which show good agreement. Hadjadj and Dussauge [8] also note that recent efforts with advanced eddy resolving methods such as LES [50, 51] and DNS [10] (shown in fig. 2.12) have been applied with significant success [8]. In fact, simulations of the same case of a supersonic compression ramp using DNS [10] and LES [50] show a very good agreement between the filtered DNS and the LES, with the LES computed at a fraction of the cost $(0.5 \% \mathrm{CPU}$ time of the DNS). These are encouraging for the use of hybrid RANS-LES for SWBLI simulations that could offer significant advances for future use in industry [8]. From Piomelli's review of hybrid RANS-LES approaches [52], they find hybrid RANS-LES methods to be most effective in flow conditions that facilitate the rapid amplification of any instability, whether due to numerical or natural causes. These include well-defined separations, concave surfaces and adverse pressure gradients where the eddy generation at the RANS-LES interface is greatly increased. This reduces the adverse effects of unphysical phenomena that can occur at this interface (the so called grey area, where RANS and LES overlap) and accurate results can be obtained. SWBLIs therefore seem particularly suited to hybrid RANSLES with shocks imposing a strong instability on the boundary layer.

Regarding RANS, Knight et al. [49], Zheltovodov [47] and Knight and Degrez [48], the latter of whom studied 13 configurations and 37 computations by 18 participants, all conclude that in general, RANS methods perform well at predicting mean surface pressure and heat transfer, and primary separation location for weak to moderate $2 \mathrm{D}$ 


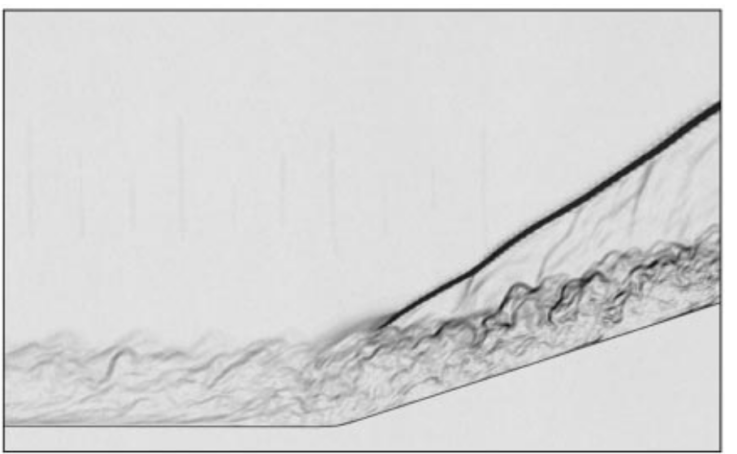

(a) Instantaneous DNS

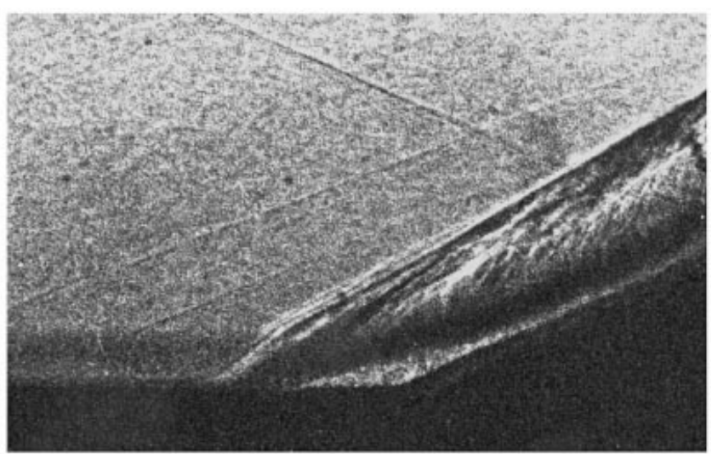

(b) Experimental Schlieren

Fig. 2.12 Comparisons between DNS and experimental flow fields of the shock over a compression ramp by Adams [10]

and 3D SWBLIs. Moreover, little variation in turbulence models is observed. Dolling [43] attributes this to the triple deck structure of these flows, where surface pressure results from the interactions between the inviscid second and third deck, and thus is not effected by turbulence model choice. This also gives further motivation for hybrid RANS-LES. However, for strong interactions, computed surface pressure, heat transfer distributions, and location of primary separation, show significant disagreement with experiment. Moreover, they find that RANS calculations failed to capture the high level of unsteadiness in the shock system and the appearance of secondary separation, and no RANS calculation provided a prediction of rms fluctuating surface pressure and heat transfer. The main cause of inaccuracy could be related to the low-frequency unsteadiness of the shock, which is not reproduced by steady RANS approaches [43, 51]. Hadjadj and Dussauge [8] also found that although significant progress has been made on SWBLI RANS prediction, models fail to capture important characteristics like high levels of shock unsteadiness and subsequent pressure fluctuations, motivating the need for further work. In addition, they state that resolving the interactions between various energy scales and flow anisotropy is needed to represent SWBLIs, which may pose difficult for isotropic eddy viscosity based turbulence models. Loyau et al. [53] also find that since the interaction is close to the wall, the turbulence here is highly anisotropic and subjected to a complex strain field, especially in the presence of separation. Moreover, EVM's characterisation of the turbulence using a single length scale also lose their validity in separated flows [43].

Loyau et al. [53] investigated a number of nonlinear eddy viscosity formulations for modelling turbulence in shock-affected flows to account for anisotropy. The models were implemented in a $k-\epsilon$ turbulence modelling framework, and were applied to 
two transonic bump flows based on the experiments of Delery et al. [54] and Bachalo and Johnson [44]. Their findings showed that nonlinear models are able to return significantly stronger SWBLI, much closer to experimental measurements, compared to the standard linear variants that significantly under-predict the strength of the interaction. However, they do note a strong sensitivity to the choice of nonlinear model with results varying considerably depending of the details of the model and approach to its calibration. Nevertheless, they find that none of the models give a full accord with experiment. Even the best performing nonlinear model in the shock, found to be that of Apsley and Leschziner [55], failed to correctly predict the post interaction flow following reattachment, although returned the closest agreement in respect of the shock induced separation point.

For eddy resolving simulations of shock affected flows, shock capturing methods are particularly important for numerical accuracy, where numerical dissipation presents a challenge. This arises from the need to apply significant numerical smoothing around shocks to dissipate local numerical errors created by the high flow gradients here, while maintaining low smoothing in all other parts of the flow. This is particularly important for eddy resolving simulations, so as to not apply excessive smoothing around turbulent fluctuations. Even modern low-dissipative, high order shock capturing schemes are still too dissipative for capturing the fine scale turbulence and flow discontinuities around shocks [8]. Nevertheless, the addition of numerical dissipation cannot be avoided in shocked flows, and therefore methods to accommodate this have been proposed [51, 56, 57]. Jameson et al. [56] proposed a pressure based shock sensor that aimed to detect shocks based on regions of high pressure gradient. However, this sensor was shown to take large values not only in presence of shocks, but also in the presence of turbulent fluctuations $[57,58]$. Ducros et al. [57] proposed a modification to this by introducing a new sensor developed to be used in conjunction with the classical Jameson sensor. The Ducros sensor is a function of vorticity and has been demonstrated to easily distinguish a turbulent fluctuation from a shock $[51,57,58]$. This modification has greatly improved LES of flows with shocks.

It may therefore seem that RANS methods are disappointing for SWBLI predictions, and persuing them further may be unfruitful. However, from Dolling [43], RANS codes have seen a high investment in recent times, driven by engineering needs for rapid predictive capabilities. Therefore, even if critical physics are missing, it is still necessary to benchmark RANS codes to know how they perform in specific flows, and what is reliable and what is not [43]. Using the deficiencies in RANS code as a motivation for LES is understandable, but it is important to remember that RANS 
codes do give adequate performance for many applications. Moreover, LES for flows in general, and SWBLIs in particular, is still in its infancy, and will take many years before these become common practice. Jameson [59] notes that while LES cannot be practically used at this time, it does provide an improved insight into the physics of turbulent flows which can in turn help develop more comprehensive and reliable turbulence models. As such, further calibrations and tuning of RANS models are very much a necessity [43].

\subsubsection{Transitional SWBLIs}

Most of the work on SWBLIs done over the past 50 years has been in fully developed turbulent flows, since most practical applications are at transonic and low supersonic speeds with large Re, where turbulent flow is the norm [60]. In fact, for most cases, transitions trips are used in experimental works to ensure transition location is fixed, and downstream interactions are fully turbulent [11]. As a result, transitional SWBLIs have received little attention and Murphree et al. [60] state that our current understanding of transitional SWBLIs is extremely poor, and that at this stage, simulations cannot be used with any measure of confidence to predict such flows. The boundary layers on intake lips at incidence are believed to be in a transitional state at the point of SWBLI, furthered by the effects of laminarisation, and so increased understanding of transitional SWBLIs is essential for future intake designs.

Transitional SWBLIs occur when an oncoming boundary layer flow is in a laminar or transitional state somewhere during the SWBLI. This generates a complex interaction, where on the one hand the shock acts as a perturbation to trigger the boundary layer to turbulence upstream of where it naturally would, and on the other, the transition to turbulence within the interaction effects the phenomenon through promoting momentum exchanges [20]. The shock interaction is stronger than a turbulent one, since a transitional boundary layer is much less resilient to the large shock imposed pressure gradient [11]. As a result, the flow can separate prematurely, with a larger extent of separation, leading to increased performance and stability issues [20]. The shock interaction can also cause the position of transition to become unstable, and this must be taken into account to avoid flutter problems [11]. It is also know that unsteady shock oscillations are more prominent here, which may be of concern in shorter intakes [11].

In addition to a lack of applications, shortage of research on transitional SWBLIs can also be attributed to the formidable challenge they pose to both experiment and computations [60]. Predictive tools must be able to provide for accurate laminar 
turbulent boundary behaviour (i.e. transition) in order to reproduce this flow well, yet studies have shown that accurate prediction of transition to turbulence in supersonic boundary layers is a goal yet to be reached. Even repeatability of transitional flow experiments are extremely challenging, where small changes in some flow or geometric parameter can shift transition position significantly from run to run, as found by Dolling [43].

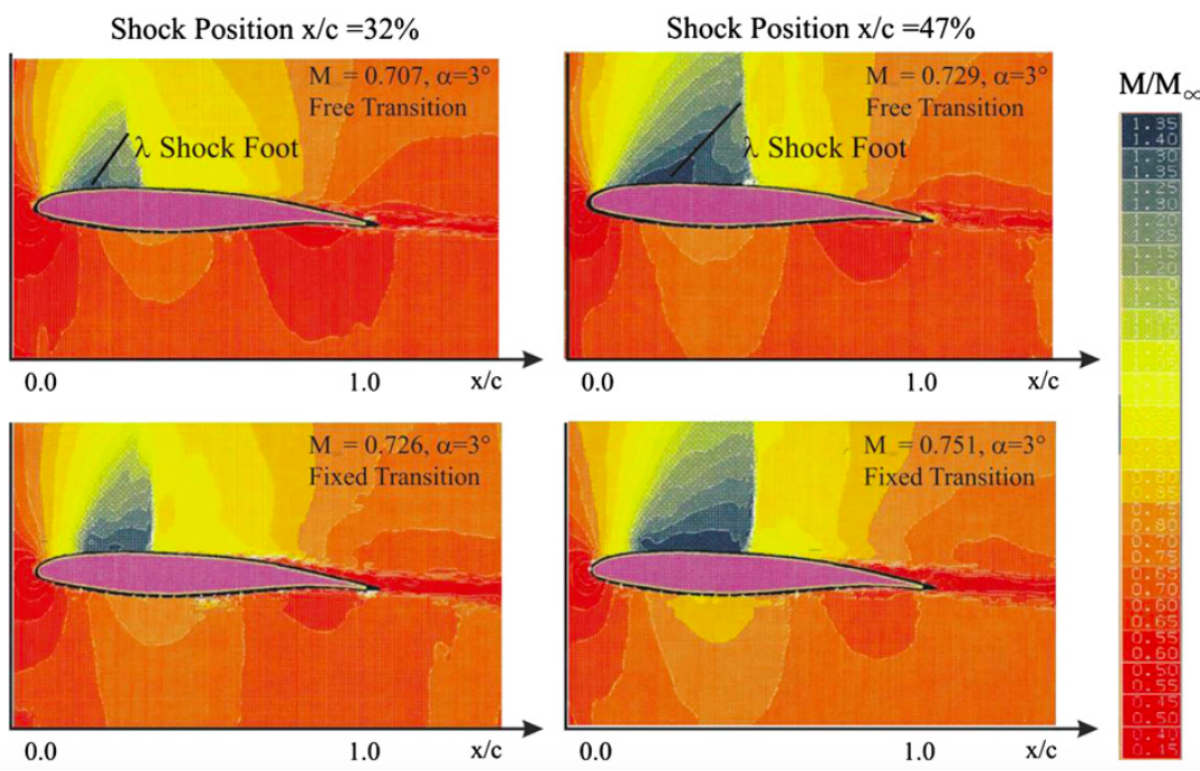

Fig. 2.13 Mach number distributions for a transonic aerofoil under free and fixed transition, and at two shock locations from Becker at al. [11]

Nevertheless, recent improvements in non intrusive experimental measurement techniques, such as particle image velocimetry (PIV) and planar laser scattering (PLS) have allowed some of the first studies on transitional SWBLIs to be conducted, in not only measuring the interaction, but also characterising the incoming boundary layer [43], such as that by Murphree et al. [60]. However, detailed quantitative measurements for CFD validations still leave a lot to be desired.

Fig. 2.13 shows Mach number distributions for a transonic aerofoil under free and fixed transition, and at two shock locations from Becker at al. [11]. With free transition, the flow decelerates and separates much earlier over the suction side of the aerofoil, as opposed to the fixed transition (turbulent) case which gives a smaller $\lambda$-shock foot, and a more vertical shock.

Becker at al. [11] conducted a numerical investigation on transitional SWBLIs on a fan blade. The freestream turbulence here is very low, which can lead to laminar/transitional boundary layers in the shock region. 3D RANS computations were 
carried out, using the SA model in combination with a transition model of AbuGhanam Shaw. Results showed a considerable difference in the blade characteristics between the fully turbulent and transitional computations, especially at low Re. The main differences were caused by significantly different shock positions between the cases and premature boundary layer separation for the transition case.

\subsection{Summary}

The literature review has highlighted many aspects of aero engine intake flows, and SWBLIs in general, that are in need of further work, or have received little attention. A summary of the main findings is given below.

- Although described in some literature, we find no work either experimentally or computationally that investigates transonic high incidence lip flows. If these phenomena are observed, they are often not the focus of research and so no useful available data exists to the authors knowledge.

- Flow phenomena such as boundary layer acceleration and curvature are important features of intake flows, although most turbulence models in their standard forms are insensitive to these without modification.

- RANS computations of SWBLIs are in significant need of improvement.

- Hybrid RANS/LES methods show promise for the simulation of SWBLIs and could offer significant advantages as a middle ground between RANS and LES.

- We find a lack of work that investigates transitional SWBLIs both experimentally and computationally, which are a prominent intake lip flow feature. As a result, CFD lacks validation data. High quality quantitative measurements are needed here that include characterisation of the boundary layer, velocity profiles and turbulence data (i.e. Reynolds stress profiles). 


\subsection{Project Strategy}

The multifaceted intake flow makes direct evaluation of CFD methods challenging. The coupled nature of flow features means it is difficult to identify regions in which schemes struggle, thus making development of corrections difficult. Shock location for example is highly dependant on the properties of the boundary layer both upstream and downstream of the shock, which in turn depend on the shock strength. Discrepancies in the flow here make it difficult to determine which one of these features is being inaccurately predicted.

On that account, the project strategy will first involve validating the HYDRA CFD code and improvements against two simplified canonical test cases. These represent a breakdown of the intake flow physics to allow development and testing of model improvements. The first is a subsonic wall mounted hump case that will allow study of streamwise curvature, boundary layer acceleration and flow separation size. Wall resolved LES will be run, exploring various SGS models and their performance for this flow. RANS modifications will also be tested. From this, a hybrid RANS-LES method will be proposed and validated.

The simulation methods developed for the subsonic hump will then be extended to an axisymmetric transonic bump case. This will introduce a shock and SWBLI and enable the prediction of shock strength and location to be validated.

Finally, the methods developed for the test cases will then be applied to the intake rig model, with the aim to provide a recommended simulation strategy for this flow.

An outline for the remainder of this thesis follows. Chapter 3 details the numerical methods and models used in this work. This includes description of code modifications and solver details. Chapter 4 and chapter 5 investigate simulations of the subsonic and transonic canonical hump cases respectively. Description and simulations of the intake rig are then presented in chapter 6 . Finally, concluding remarks and recommendations for future work are given in chapter 7 . 


\section{Chapter 3}

\section{Numerical Methods}

\subsection{Turbulence Treatments}

Turbulence is the three dimensional unsteady chaotic motion of fluid flow. Its seemingly random and fundamentally stochastic nature mean that turbulence is notoriously difficult to numerically predict, and is often considered one of the most important unsolved problems of classical physics.

The Navier-Stokes equations that govern compressible viscous fluid flow are presented below, composed of the mass (continuity), momentum and energy conservation equations respectively. These are three dimensional non-linear partial differential equations, that in their basic form, are believed to fully describe the time dependent turbulent motions.

$$
\begin{gathered}
\frac{\partial \rho}{\partial t}+\frac{\partial\left(\rho u_{i}\right)}{\partial x_{i}}=0 \\
\frac{\partial\left(\rho u_{i}\right)}{\partial t}+\frac{\partial\left(\rho u_{j} u_{i}\right)}{\partial x_{j}}=-\frac{\partial p}{\partial x_{i}}+\frac{\partial \tau_{i j}}{\partial x_{j}}+g_{i} \\
\frac{\partial E}{\partial t}+\frac{\partial\left((E+p) u_{j}\right)}{\partial x_{j}}=-\frac{\partial q_{j}}{\partial x_{j}}+\frac{\partial\left(\tau_{i j} u_{i}\right)}{\partial x_{j}}
\end{gathered}
$$

These are closed with expressions for the heat flux $q_{i}$ and an equation of state (the ideal gas equation) given by,

$$
\begin{gathered}
q_{i}=-k \frac{\partial T}{\partial x_{i}} \\
p=\rho R T
\end{gathered}
$$


The shear stress $\tau_{i j}$ is given by,

$$
\tau_{i j}=2 \mu S_{i j}=\mu\left(\frac{\partial u_{i}}{\partial x_{j}}+\frac{\partial u_{j}}{\partial x_{i}}\right)
$$

and $S_{i j}$ is the rate of strain tensor,

$$
S_{i j}=\frac{1}{2}\left(\frac{\partial u_{i}}{\partial x_{j}}+\frac{\partial u_{j}}{\partial x_{i}}\right)
$$

Note the use of Einstein notation, where repeated indices imply summation over that index. The term $\partial \tau_{i j} / \partial x_{j}$ can also be written as $\mu \partial^{2} u_{i} / \partial x_{j}^{2}$ using continuity. Note that the body force (per unit mass) term $g_{i}$ has also been included above, although this will be ignored in further derivations since it is not used in this work.

While turbulence can be fully described by the Navier-Stokes equations, computations of the entire turbulent length and time scale range by means of DNS are still far too computationally expensive for many flows of engineering interest. This leads to turbulence modelling techniques explored in the following sections.

\subsection{Reynolds-Averaged Navier-Stokes}

The most widely used approach in turbulence modelling is the application of the Reynolds averaged Navier-Stokes (RANS) equations. These filter out the unsteady turbulent motions of the flow field (and consequently the need to resolve them) and solve directly for the time-averaged steady flow instead. This negates the need for temporal resolution, and also allows 2D simulation where the time-averaged flow field under investigation can be assumed sufficiently two-dimensional. As a result, RANS provides a computationally cheap, easy to use and cost effective way of simulating real flows, and is the engineering tool of choice for most industrial applications [61].

The RANS equations are derived through a decomposition of the flow field into mean and fluctuating components, known as Reynolds decomposition [62]. Flow variables such as velocity and pressure are written as the sum of their mean and fluctuating parts as,

$$
u=\bar{u}+u^{\prime}
$$

where the use of ${ }^{-}$and ${ }^{\prime}$ denote the mean and fluctuating parts respectively. Following this approach, it is assumed that the mean of fluctuating components is zero, $\overline{u^{\prime}}=0$, and that the mean component is statistically steady, $\overline{\bar{u}}=\bar{u}$. This new definition of 
flow variables allows a time-averaged set of the Navier-Stokes equations to be derived as,

$$
\begin{gathered}
\frac{\partial\left(\bar{\rho} \bar{u}_{i}\right)}{\partial x_{i}}=0 \\
\bar{u}_{j} \frac{\partial\left(\bar{\rho} \bar{u}_{i}\right)}{\partial x_{j}}=-\frac{\partial \bar{p}}{\partial x_{i}}+\frac{\partial \bar{\tau}_{i j}}{\partial x_{j}}-\frac{\partial\left(\bar{\rho} \overline{u_{i}^{\prime} u_{j}^{\prime}}\right)}{\partial x_{j}}
\end{gathered}
$$

Where $\bar{\tau}_{i j}$ is now given by,

$$
\bar{\tau}_{i j}=2 \mu \bar{S}_{i j}=\mu\left(\frac{\partial \bar{u}_{i}}{\partial x_{j}}+\frac{\partial \bar{u}_{j}}{\partial x_{i}}\right)
$$

and $\bar{S}_{i j}$ by,

$$
\bar{S}_{i j}=\frac{1}{2}\left(\frac{\partial \bar{u}_{i}}{\partial x_{j}}+\frac{\partial \bar{u}_{j}}{\partial x_{i}}\right)
$$

We can note the presence of an additional term in the RANS momentum equation (3.10), $\bar{\rho} \overline{u_{i}^{\prime} u_{j}^{\prime}}$, that accounts for the averaged contribution of the turbulent velocity fluctuations to the mean flow. This second moment term is referred to as the Reynolds stress due to its analogy to the mean viscous stress, and its presence indicates the need for additional work to solve the equations. Attempts at deriving further equations for the second moments result in equations with even higher order moments that are themselves unknown [63]. This is the well known closure problem of turbulence, and gives rise to the field of turbulence modelling that attempts to close the RANS formulation by providing models for these unknown second moments.

The most widely used closure models are based on the eddy viscosity concept, which suggests a Boussinesq-type approximation between the Reynolds stresses and mean strain rate [61] given by,

$$
\overline{u_{i}^{\prime} u_{j}^{\prime}}=\frac{2}{3} k \delta_{i j}-2 \nu_{t} \bar{S}_{i j}
$$

where $k$ is the turbulent kinetic energy $\left(k=\overline{u_{i}^{\prime} u_{i}^{\prime}} / 2\right), \delta_{i j}$ is the Kronecker delta and $\nu_{t}$ is a turbulent eddy viscosity to be solved for. Using this formulation, it can be noted that the turbulence field is only coupled to the mean field through this turbulent eddy viscosity [61].

Over the years, numerous schemes have been proposed that attempt to model $\nu_{t}$, usually through the inclusion of additional transport equations that aim to specify turbulent velocity and length scales. However, as found in the literature review, the variability of RANS models performance for specific flow types means that a 'one 
model fits all' does not exist. In this work, the Spalart-Allmaras model [64] is chosen as the representative RANS model for investigation, and will be the focus of this work. This is due to its simplicity, popularity, and good performance for a wide variety of flow types.

\subsubsection{SA Model}

The Spalart-Allmaras (SA) model is a one-equation turbulence model that solves an additional transport equation for a viscosity like variable, $\tilde{\nu}$, known as the SA variable. Devised by Spalart and Allmaras in 1994 [64], this simple model is known for its ease of implementation, low computational cost and good performance for a wide variety of flows [61]. In some cases, it has even been shown to perform better than more advanced, two-equation models [65] and as a result, has become very popular since its inception.

The variable $\tilde{\nu}$ is solved for using the transport equation,

$$
\begin{aligned}
\frac{\partial \tilde{\nu}}{\partial t}+u_{j} \frac{\partial \tilde{\nu}}{\partial x_{j}}=c_{b 1}\left(1-f_{t 2}\right) \tilde{S} \tilde{\nu}-\left[c_{w 1} f_{w}\right. & \left.-\frac{c_{b 1}}{\kappa^{2}} f_{t 2}\right]\left(\frac{\tilde{\nu}}{d}\right)^{2} \\
+ & \frac{1}{\sigma}\left[\frac{\partial}{\partial x_{j}}\left((\nu+\tilde{\nu}) \frac{\partial \tilde{\nu}}{\partial x_{j}}\right)+c_{b 2} \frac{\partial \tilde{\nu}}{\partial x_{i}} \frac{\partial \tilde{\nu}}{\partial x_{i}}\right]
\end{aligned}
$$

from which $\nu_{t}$ is computed using the eddy viscosity relation,

$$
\begin{aligned}
\nu_{t} & =\tilde{\nu} f_{v 1} \\
f_{v 1} & =\frac{\chi^{3}}{\left(\chi^{3}+c_{v 1}^{3}\right)}
\end{aligned}
$$

and $\chi=\tilde{\nu} / \nu$. The terms $c_{b 1}\left(1-f_{t 2}\right) \tilde{S} \tilde{\nu}$ and $\left[c_{w 1} f_{w}-\frac{c_{b 1}}{\kappa^{2}} f_{t 2}\right]\left(\frac{\tilde{\nu}}{d}\right)^{2}$ in (3.14) may also be categorised as turbulence production $P(\tilde{\nu})$ and destruction $D(\tilde{\nu})$ terms respectively due to their source effects on the turbulent flow field. Description of the remaining auxiliary relations are,

$$
\begin{gathered}
\tilde{S}=S+\frac{\tilde{\nu}}{\kappa^{2} d^{2}} f_{v 2}, \quad S=\sqrt{2 s_{i j} s_{i j}}, \quad f_{v 2}=1-\frac{\chi}{1+\chi f_{v 1}}, \\
f_{w}=g\left(\frac{1+c_{w 3}^{6}}{g^{6}+c_{w 3}^{6}}\right)^{1 / 6}, \quad g=r+c_{w 2}\left(r^{6}-r\right), \quad r=\frac{\tilde{\nu}}{\tilde{S} \kappa^{2} d^{2}},
\end{gathered}
$$


where $\mathrm{d}$ is the distance to the nearest wall, and the closure constants are,

$$
\begin{array}{r}
c_{b 1}=0.1355, \quad c_{b 2}=0.622, \quad \sigma=2 / 3, \quad c_{v 1}=7.1, \\
c_{w 1}=\frac{c_{b 1}}{\kappa^{2}}+\frac{1+c_{b 2}}{\sigma} \quad c_{w 2}=0.3, \quad c_{w 3}=2, \quad \kappa=0.41 .
\end{array}
$$

As was found in the literature review, boundary layer acceleration and curvature are important features of intake flows, although most turbulence models in their standard forms are insensitive to these without modification. The SA model is therefore modified in this work to account for the effects of relaminarisation and curvature. These are described in the following sections.

\section{Relaminarisation Modification}

The standard SA model is modified to account for the effects of laminsarisation based on the work of Launder and Jones [27] and recent work by Oriji [1]. A description of the modification from Oriji [1] is presented here.

A modification of the Van Driest damping function $D_{J L}$ is proposed by Launder and Jones[27] as,

$$
D_{J L}=1-\exp \left(-\frac{y^{+}}{A^{+}(L)}\right)
$$

where $A^{+}$, originally a constant defined by Van Driest [66] as $A^{+}=26$, is modified to a variable function of $L=K_{S} C_{f}^{-3 / 2}$. It is recalled that $K_{S}$ is the acceleration parameter used to discern laminarisation, previously defined in equation (2.1), and $C_{f}$ is the skin friction coefficient. $A^{+}$is then given by Launder and Jones [27] as,

$$
\begin{aligned}
& A^{+}=26 \\
& \left.L \leq 1.9 \times 10^{-3}\right\} \\
& A^{+}\left(K_{S}\right)=11+\left(7.9 \times 10^{3}\right) L \quad L \geq 1.9 \times 10^{-3} \text {. }
\end{aligned}
$$

Since $\chi=\kappa y^{+}$from around the wall to the log layer in the SA model, $\chi$ in (3.16) is modified to $\tilde{\chi}$ as,

$$
\tilde{\chi}=\frac{\chi}{\chi_{A}}
$$

where $\chi_{A}=\max \left(1.0, \kappa A^{+}\left(K_{S}\right)\right)$. This allows recovery of the standard SA model where $K_{S}$ is small, returning $\chi_{A}=1$ and $\tilde{\chi}=\chi$. Following this approach, regions of high $K_{S}$ would tend to reduce $\tilde{\chi}$, hence decreasing $f_{v 1}$ and therefore reducing the 
turbulent viscosity $\nu_{t}$ in this zone. $K_{S}$ is computed using,

$$
\begin{aligned}
K_{S} & =\frac{\nu}{u_{e}^{2}}\left(u \frac{d u_{e}}{d x}+v \frac{d u_{e}}{d y}+w \frac{d u_{e}}{d z}\right) \frac{1}{v_{t}} \\
u_{e} & =M_{i s} a \\
v_{t} & =\sqrt{u^{2}+v^{2}+w^{2}} \\
M_{i s} & =\sqrt{\left[\left(\frac{P_{T}}{P}\right)^{1 / 3.5}-1\right] / 0.2}
\end{aligned}
$$

where $u_{e}$ is the boundary layer edge velocity, $M_{i s}$ is the isentropic mach number, $a$ is the speed of sound, $v_{t}$ is the tangential streamline velocity, $P_{T}$ is the freestream total pressure and $P$ is the local static pressure.

\section{Rotation/Curvature Correction}

To account for the effects of curvature, the SA model Rotation/Curvature (SARC) correction proposed by Spalart and Shur [33] is implemented. A description of the correction from Zhang and Yang [32] is presented here.

The production term $c_{b 1}\left(1-f_{t 2}\right) \tilde{S} \tilde{\nu}$ in the SA transport equation (3.14) is multiplied by a new rotation function $f_{r 1}$ defined as,

$$
f_{r 1}\left(r^{*}, \tilde{r}\right)=\left(1+c_{r 1}\right) \frac{2 r^{*}}{r^{*}+1}\left[1-c_{r 3} \tan ^{-1}\left(c_{r 2} \tilde{r}\right)\right]-c_{r 1}
$$

where the factor to account for the effects of rotation and curvature is,

$$
\tilde{r}=\frac{2 W_{i k} S_{j k}}{D^{4}}\left[\frac{D S_{i j}}{D t}+\left(\epsilon_{i m n} S_{j n}+\epsilon_{j m n} S_{i n}\right) \Omega_{m}^{\prime}\right]
$$

where $\epsilon_{i m n}$ is the Levi-Civita symbol. The remaining variables and functions are defined below.

$$
\begin{array}{r}
r^{*}=S / \Omega, \quad S=\sqrt{2 S_{i j} S_{i j}}, \quad \Omega=\sqrt{2 W_{i j} W_{i j}}, \\
S_{i j}=\frac{1}{2}\left(\frac{\partial u_{i}}{\partial x_{j}}+\frac{\partial u_{j}}{\partial x_{i}}\right), \quad W_{i j}=\frac{1}{2}\left[\left(\frac{\partial u_{i}}{\partial x_{j}}+\frac{\partial u_{j}}{\partial x_{i}}\right)+2 \epsilon_{m j i} \Omega_{m}^{\prime}\right] \\
D^{2}=\frac{1}{2}\left(S^{2}+\Omega^{2}\right), \quad c_{r 1}=1, \quad c_{r 2}=12, \quad c_{r 3}=0.6
\end{array}
$$

where $S$ and $\Omega$ are the strain rate and vorticity magnitudes respectively. It can be noted in equation (3.25) the presence of the material derivative $D S_{i j} / D t$. The need to compute this increases the complexity of the programming and computational cost of 
the SARC model, with Zang and Yang [32] reporting a 20\% increase in computational time. Simpler alternative methods that circumvent this have been proposed [32], although the original method by Spalart and Shur [33] is used here.

\subsection{Large Eddy Simulations}

Unlike RANS methods that model the entirety of unsteady turbulent motions, LES increases the fidelity of simulations by only modelling the small scales. The large eddies, that contain information about the geometry and dynamics of the specific problem under investigation, are explicitly resolved in space and time [43]. This significantly increases the representation of the flow and as a result, makes LES a superior simulation technique. However, the need to resolve these unsteady three dimensional large scales, including fine near wall turbulent structures present in most applications of interest, mean that LES is still prohibitively expensive for use in industry. Simulations often require use of large parallel computing facilities to obtain results in reasonable time frames, and are usually limited to simulations of individual components and flow conditions. Nevertheless, the richness of LES data enables valuable insights to complex flow physics that, as well as increasing understanding of the flow, can feed back into improving RANS models.

Despite its computational expense, LES is considered to have a simple formulation. The small scales of the flow (regarded as having a universal character) are modelled using a SGS model, which is often a simple empirical relation, as opposed to the complexity of RANS model transport equations.

The LES formulation is derived by applying a filtering operation to the NavierStokes equations. In a process similar to Reynolds decomposition, this is done by first decomposing the flow variables into filtered (resolved) and sub-filtered (unresolved) parts as,

$$
\phi=\langle\phi\rangle+\hat{\phi}
$$

where the use of $\langle\cdot\rangle$ and $\hat{\cdot}$ denote the filtered and sub-filtered parts respectively. Following this, a filtered form of the Navier-Stokes equations are obtained as,

$$
\begin{gathered}
\frac{\partial\langle\rho\rangle}{\partial t}+\frac{\partial\left(\langle\rho\rangle\left\langle u_{i}\right\rangle\right)}{\partial x_{i}}=0 \\
\frac{\partial\left(\langle\rho\rangle\left\langle u_{i}\right\rangle\right)}{\partial t}+\frac{\partial\left(\langle\rho\rangle\left\langle u_{j}\right\rangle\left\langle u_{i}\right\rangle\right)}{\partial x_{j}}=-\frac{\partial\langle p\rangle}{\partial x_{i}}+\frac{\partial\left\langle\tau_{i j}\right\rangle}{\partial x_{j}}-\frac{\partial\langle\rho\rangle \tau_{i j}^{r}}{\partial x_{j}}
\end{gathered}
$$


Where $\left\langle\tau_{i j}\right\rangle$ is given by,

$$
\left\langle\tau_{i j}\right\rangle=2 \mu\left\langle s_{i j}\right\rangle=\mu\left(\frac{\partial\left\langle u_{i}\right\rangle}{\partial x_{j}}+\frac{\partial\left\langle u_{j}\right\rangle}{\partial x_{i}}\right)
$$

and $\left\langle s_{i j}\right\rangle$ by,

$$
\left\langle s_{i j}\right\rangle=\frac{1}{2}\left(\frac{\partial\left\langle u_{i}\right\rangle}{\partial x_{j}}+\frac{\partial\left\langle u_{j}\right\rangle}{\partial x_{i}}\right)
$$

The term $\tau_{i j}^{r}$ in the filtered momentum equation (3.28) is referred to as the residual stress tensor. This accounts for the effects of the sub-filtered flow variables and must be modelled. Again, a Boussinesq-type approximation is used here, giving,

$$
\tau_{i j}^{r}=\frac{1}{3} \frac{\partial u_{k}}{\partial x_{k}} \delta_{i j}-2 \nu_{s g s}\left\langle s_{i j}\right\rangle
$$

where $\nu_{\text {sgs }}$ is a sub grid scale viscosity solved for using an SGS model.

\subsubsection{Smagorinsky-Lilly Model}

Various SGS models have been proposed over the years. One of the the earliest and simplest models is the Smagorinsky-Lilly model [67] [68]. The model calculates $\nu_{\text {sgs }}$ as,

$$
\nu_{\text {sgs }}=\left(C_{S} \Delta_{v o l}\right)^{2} S
$$

where $C_{s}=0.165$ is the Smagorinsky model constant and $S$ is the strain rate magnitude $\left(S=\sqrt{2 s_{i j} s_{i j}}\right)$, also known as the model's differential operator. $\Delta_{v o l}$ is the filter width used to locally determine the filtering between the resolved and unresolved (modelled) turbulent scales. This is defined as the cube root of the local grid cell volumes, $\Delta_{v o l}=\sqrt[3]{\Delta_{x} \Delta_{y} \Delta_{z}}$, where $\Delta_{x}, \Delta_{y}$ and $\Delta_{z}$ are the cell dimensions. It follows that the scales below this grid width, the sub-grid scales, are those that the SGS models. LES grids must therefore be carefully designed to obtain sufficient resolution of required turbulent flow structures. As the grid is refined $\left(\Delta_{v o l}\right.$ decreased) more of the turbulent scale range is directly resolved, and less of the small scales are modelled. It can be noted that continuing to refine the grid down the Kolmogorov scales allows LES to approach DNS, where the entire range of turbulent scales are directly resolved.

The simplicity and ease of implementation of the Smagorinsky-Lilly model has made it popular for LES. However, the model does have its drawbacks. In real wall bounded flows, turbulent motions are damped out in the viscous sub-layer of the boundary layer. To simulate this correctly, $\nu_{s g s}$ should tend to zero here. However, 
the Smagorinsky-Lilly model fails to naturally model this effect. High aspect ratio cells found near the wall have a large $\Delta_{v o l}$ and as a result, the model returns an excessively high $\nu_{\text {sgs }}$. To resolve this, the Smagorinsky-Lilly model uses wall damping, whereby $C_{s} \Delta_{v o l}$ is modified to

$$
C_{s} \Delta_{v o l}=\min \left(C_{s} \Delta_{v o l}, \kappa d\right)
$$

where $\kappa=0.41$ and $d$ is the distance to the wall. This modification limits $\nu_{\text {sgs }}$ near the wall to much more suitable values.

While this does improve results dramatically, this is an ad hoc approach, and can also present difficulties for complex geometries [69].

Another drawback of the Smagorinsky-Lilly model is its behaviour in pure shear flow. The model returns a large $\nu_{\text {sgs }}$ here, derived from the Smagorinsky-Lilly model's operator being based only on the strain rate $S$. This always give a non-zero value of $\nu_{s g s}$ if a velocity gradient exists. This is not the case for pure shear, which contributes very little to energy dissipation [69]. A consequence of this is the Smagorinsky-Lilly model's inability to reproduce laminar flow and transition well. Laminar flow consists of almost pure shear, and the excessively large $\nu_{\text {sgs }}$ produced by the Smagorinsky-Lilly model here has the effect of dissipating linearly unstable waves that contribute to the transition to turbulence [69].

The Smagorinsky-Lilly model is the default SGS model in HYDRA. In addition to this, two other SGS models have been implemented into HYDRA in this work to address some of the Smagorinsky-Lilly model shortfalls. They are the WALE and Sigma $(\sigma)$ models. These are described in the following sections.

\subsubsection{WALE Model}

The Wall-Adapting-Local-Eddy-viscosity (WALE) model is an SGS model proposed by Nicoud et al. [69]. The model proposes a differential operator not only based on the strain rate, but the rotation rate too,

$$
D_{w}=\frac{\left(s_{i j}^{d} s_{i j}^{d}\right)^{3 / 2}}{\left(s_{i j} s_{i j}\right)^{5 / 2}+\left(s_{i j}^{d} s_{i j}^{d}\right)^{5 / 4}}
$$

where,

$$
\begin{array}{r}
s_{i j}^{d} s_{i j}^{d}=\frac{1}{6}\left(s^{2} s^{2}+\Omega^{2} \Omega^{2}\right)+\frac{2}{3} s^{2} \Omega^{2}+2 I V_{s \Omega} \\
s^{2}=s_{i j} s_{i j}, \quad \Omega^{2}=\Omega_{i j} \Omega_{i j}, \quad I V_{s \Omega}=s_{i k} s_{k j} \Omega_{j l} \Omega_{l i}
\end{array}
$$


The model also has its own constant, $C_{w}=0.5$. Unlike the Smagorinsky-Lilly model, the WALE model naturally goes to zero near the wall without the need for a wall damping function, and also goes to zero in pure shear flows.

\subsubsection{Sigma Model}

Nicoud et al. proposed an advancement to the WALE model, the Sigma $(\sigma)$ SGS model [70]. The model proposes a new differential operator based on the singular values of the resolved velocity gradient tensor, $\sigma_{1}, \sigma_{2}$ and $\sigma_{3}$ as,

$$
D_{\sigma}=\frac{\sigma_{3}\left(\sigma_{1}-\sigma_{2}\right)\left(\sigma_{2}-\sigma_{3}\right)}{\sigma_{1}^{2}}
$$

The model also has its own constant, $C_{\sigma}=1.35$. As well as naturally going to zero near the wall and in pure shear flows, the sigma model also correctly tends to zero in solid rotation and axisymmetric compression/expansion flows.

\subsection{Hybrid RANS-LES}

As has been discussed in the literature review, it is often desired to combine the advantages of RANS and LES. In this work, hybrid RANS-LES simulations are carried out using an approach similar to Davidson and Peng [37] and Oriji et al. [1]. A RANS layer is used in the near wall region $y^{+} \leq 60$ using the SA model. LES is used in the remaining part of the flow with the SGS models described in the previous sections. The same continuity and momentum equations are solved throughout the domain, the only difference being that the turbulent viscosity $\left(\nu_{t}\right)$ is taken from the SA model in the RANS region $\left(\nu_{\text {rans }}\right)$, and from the SGS model in the LES region $\left(\nu_{\text {sgs }}\right)$. The two turbulent viscosities are smoothly blended at the RANS-LES interface over a distance of $y^{+}=10$ using a tanh blending function,

$$
\nu_{t}= \begin{cases}\nu_{\text {rans }} & \text { if } y^{+}<50 \\ \phi \nu_{\text {sgs }}+(1-\phi) \nu_{\text {rans }} & \text { if } 50<y^{+}<60 \\ \nu_{\text {sgs }} & \text { if } y^{+}>60\end{cases}
$$

where,

$$
\phi=0.5\left[1+\tanh \left(\frac{d-h}{w}\right)\right]
$$


where $d$ is the distance to the wall. $h$ and $w$ are chosen so that $\nu_{\text {rans }}$ and $\nu_{\text {sgs }}$ blend smoothly at the interface.

Unlike Oriji et al. [1] that used a frozen RANS solution obtained from a precursor simulation, both the RANS and LES domains in this work are resolved together and updated every time step. However, aggressive first-order numerical smoothing is used in the RANS layer to dissipate any resolved turbulent fluctuations here. This is to reduce the so called double accounting of turbulence where the RANS zone has both modelled and resolved scales, the latter being problematic and a well-known defect of hybrid RANS-LES methods [2, 19].

Using a fixed RANS layer size $\left(y^{+} \leq 60\right)$ also makes the RANS-LES interface independent of the mesh. The distance $y^{+}=60$ is evaluated based on an averaged shear stress in the target zone, and is constant on the wall.

\subsection{HYDRA}

Simulations in this work are carried out using the Rolls-Royce unstructured compressible flow solver, HYDRA [71]. The solver is second order in both space and time. It offers multi-grid capability for steady calculations, enabling accelerated simulation convergence, with coarse grids automatically generated using an element collapsing algorithm. Its second order nature allow computations to benefit from efficient parallel capabilities for distributed memory machines, whereby communications between processors is reduced compared to higher order codes. Its unstructured aspect also makes it particularly suited to the complex geometries found in turbo-machinery for which it was designed.

For unsteady calculations, an explicit 5 stage Runge-Kutta temporal integration scheme is used, with the time step, $\Delta t$, defined using a user specified CourantFriedrichs-Lewy (CFL) number.

\subsubsection{Spacial Discretisation}

HYDRA is a node-centred solver and features an edge based finite volume spacial discretisation scheme. Flow variables stored at each grid node are calculated through flux calculations following the control volume approach. This is initialised by constructing dual median control volumes around each grid node formed by joining the centroids of neighbouring cells as illustrated around an arbitrary grid node in figure 3.1. Fluxes entering each control volume are calculated by taking the surface integral of the fluxes 
through each control volume face. These are calculated at the midpoint of all the grid edges $e_{i j}$ connected to the node, through the control volume face associated with that edge.

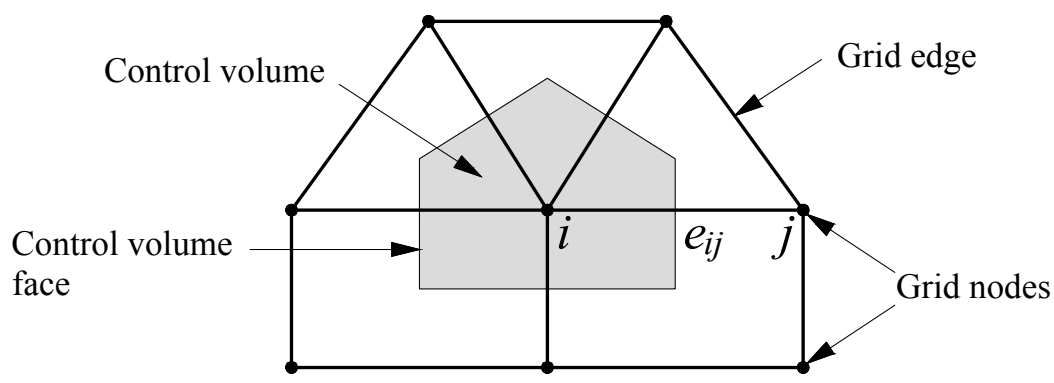

Fig. 3.1 HYDRA's dual median control volume around an arbitrary grid node

The calculation of the fluxes at each edge are split into viscous and inviscid components,

$$
F_{i j}=F_{i j}^{V}+F_{i j}^{I}
$$

where $F_{i j}^{V}$ and $F_{i j}^{I}$ are the viscous and inviscid parts respectively. The inviscid fluxes are calculated using the method of Roe [72] to solve the Riemann problem encountered at the interface of adjacent control volumes. This can be considered a second order central differencing method, smoothed by some upwinding based on the nodal variables each side of the control volume face [35],

$$
F_{i j}^{I}=\frac{1}{2}\left(F_{i j}^{I}\left(Q_{i}\right)+F_{i j}^{I}\left(Q_{j}\right)\right)-\frac{1}{2}\left|A_{i j}\right|\left(Q_{R}-Q_{L}\right)
$$

where $\left|A_{i j}\right|$ is the flux jacobian $\left(\left|A_{i j}\right|=\partial F^{I} / \partial Q\right)$ and $Q_{i}$ and $Q_{j}$ are the associated nodal variables. The dissipative second order smoothing term (the last term in equation (3.40)) is required to maintain a stable, error free solution, as the second order central differencing scheme is prone to numerical instability [35]. This term is approximated in HYDRA by introducing a second order smoothing constant $\epsilon_{2}$ to give,

$$
\frac{1}{2}\left|A_{i j}\right|\left(Q_{R}-Q_{L}\right) \approx \epsilon_{2} \frac{1}{2}\left|A_{i j}\right|\left(\nabla_{j}^{2} Q-\nabla_{i}^{2} Q\right)
$$

where $\nabla_{i}^{2}$ and $\nabla_{j}^{2}$ are undivided Laplacians evaluated at the respective nodal locations [19].

By directly scaling the level of numerical smoothing, the specification of $\epsilon_{2}$ is an important consideration in HYDRA when running eddy resolving simulations (i.e. LES or hybrid RANS/LES). Insufficient numerical smoothing can cause dispersive errors 
in the solution. These errors present themselves as unphysical point-wise oscillations (or "wiggles") in the flow field that can disturb or otherwise influence the flow under investigation. In transitional flow for example, these disturbances can lead to premature transition to turbulence, greatly affecting the reliability of results. Sufficient numerical smoothing is needed to suppress these.

Conversely, excessive numerical smoothing can lead to excessive dissipation of physical turbulent flow structures. In a separated flow for example, excessive damping of turbulence can lead to a delay in flow reattachment. It is therefore critical to find a balance when selecting the value of $\epsilon_{2}$ to have the numerical smoothing as low as possible, while maintaining a stable solution free of dispersion error.

In this regards, the selection of $\epsilon_{2}$ in this work is done by reducing $\epsilon_{2}$ gradually until the first signs of dispersion error are seen in the region of interest. $\epsilon_{2}$ is then increased marginally to achieve a dispersion free solution with minimum numerical dissipation. The solution is monitored throughout the run to ensure no dispersion errors appear. This is done for all LES and hybrid RANS/LES simulations.

The treatment of very high gradient flow regions such a shocks, a focus of this work, is of particular importance with regards to numerical stability. Dispersive errors near these high gradient areas can be seen as unphysical Gibbs-like ringing around the shock region [35]. The second order smoother is inadequate to suppress the errors here. HYDRA therefore includes a more aggressive first-order smoother in the inviscid flux calculation to handle this type of flow. This is controlled using the Jameson sensor [56], a simple pressure switch,

$$
\Psi=\min \left(\epsilon_{3}\left|\frac{p_{j}-p_{i}}{p_{i}+p_{j}}\right|^{2}, 1\right)
$$

where $\epsilon_{3}$ is a smoothing constant. The pressure switch $\Psi$ has a value close to one in regions of strong pressure gradient, and a value close to zero in regions of low pressure gradient. This allows HYDRA to adequately capture shocks and only apply the lower order smoother around these high gradient regions, while maintaining second order accuracy in all other smooth areas of the flow. The inviscid flux equation can thus be written as,

$$
F_{i j}^{I}=\frac{1}{2}\left(F_{i j}^{I}\left(Q_{i}\right)+F_{i j}^{I}\left(Q_{j}\right)\right)-(1-\Psi) \epsilon_{2} \frac{1}{2}\left|A_{i j}\right|\left(\nabla_{j}^{2} Q-\nabla_{i}^{2} Q\right)+\Psi\left(Q_{j}-Q_{i}\right)
$$

where the last term is the lower order smoothing term.

As can be seen from equation (3.43), the standard smoothing field in HYDRA is 
a mixture of first and second order smoothing controlled by $\Psi$. While this is required for shock flow, subsonic flows without shocks can benefit from a pure second order accurate solution. To achieve this, $\epsilon_{3}=0$ is set for all subsonic simulations in this work.

To complete the flux calculations, the remaining viscous fluxes, $F_{i j}^{V}$, are calculated. These are achieved economically by approximating them at the midpoint of all the edges $e_{i j}$ connected to the node [35]. Again, this is where the control volume faces intersect the edge, and is also a central difference scheme, thereby maintaining consistency with the inviscid flux calculation. This is done by estimating the gradients of the flow variables using a simple average of the values at the endpoints of the edge,

$$
\overline{\nabla Q_{i j}}=\frac{1}{2}\left(\nabla Q_{i}+\nabla Q_{i}\right)
$$

In regions where viscous terms dominate, such as boundary layers, the scheme can become unstable due to the generation of instabilities by high frequency variations [35]. To solve this, the scheme is modified to,

$$
\nabla Q_{i j}=\overline{\nabla Q_{i j}}-\left(\overline{\nabla Q_{i j}} \cdot \delta s_{i j}-\frac{Q_{i}-Q_{j}}{\left|x_{i}-x_{j}\right|}\right) \delta s_{i j}
$$

where,

$$
\delta s_{i j}=\frac{x_{i}-x_{j}}{\left|x_{i}-x_{j}\right|}
$$

\subsubsection{Ducros Sensor}

While the standard shock sensor of Jameson [56] used in HYDRA is able to capture shocks, the sensor has been shown to take large values in the presence of turbulent fluctuations too $[57,58]$. This is destructive to eddy resolving simulations as it leads to the solver applying aggressive first-order numerical dissipation to resolved turbulent fluctuations.

Ducros et al. [57] proposed a solution to this by introducing a new sensor developed to be used in conjunction with the classical Jameson sensor. The Ducros sensor is a function of vorticity and has been demonstrated to be able to sucessfully distinguish a turbulent fluctuation from a shock $[51,57,58]$. This is a simple, local, sensor and does not require any prior knowledge of the shock position or flow. The sensor computes a local variable,

$$
\Phi=\frac{(\nabla \cdot u)^{2}}{(\nabla \cdot u)^{2}+(\omega)^{2}+\epsilon}
$$


where $\omega$ is the vorticity $(\omega=\nabla \times u)$ and $\epsilon=10^{-30}$ is a small positive real number chosen to prevent numerical divergence when both $\nabla \cdot u$ and $\omega$ are zero. $\Phi$ is multiplied by the standard sensor $\Psi$ of equation (3.42) to form the new switch. The Ducros sensor is implemented into HYDRA in this work.

\subsection{Turbulent Boundary Layers}

The canonical flow cases simulated in this work require inflow turbulent boundary layers (TBL) to replicate experimental flow conditions. These were generated in a precursor simulation using the Lund recycling/rescaling technique [12]. This was kindly carried out by Dr. Naqavi at the University of Cambridge for the purpose of this work. Full details of this method can be found in [12], although a brief description is given here.

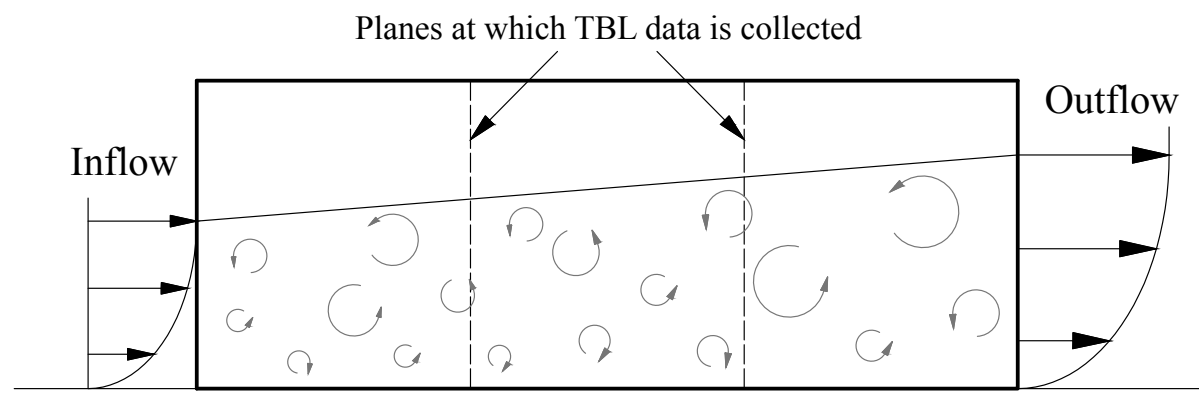

Fig. 3.2 Schematic showing the collection of TBL data using the Lund recycling/rescaling technique [12]

The method generates turbulent data from an auxiliary simulation of a zero pressure gradient boundary layer. As shown in figure 3.2, the boundary layer is streamwise growing. The simulation generates its own inflow conditions through a sequence of operations where the velocity field at a downstream station is rescaled and re-introduced at the inlet [12]. From this, instantaneous planes of velocity are extracted at stations that correspond to required TBL momentum thickness Reynolds numbers, $R e_{\theta}$. These planes are then constructed to form the three-dimensional, time-series turbulent data sets. The method is shown to produce realistic TBLs which yield statistics that are in good agreement with both experimental data and results from DNS [12]. 


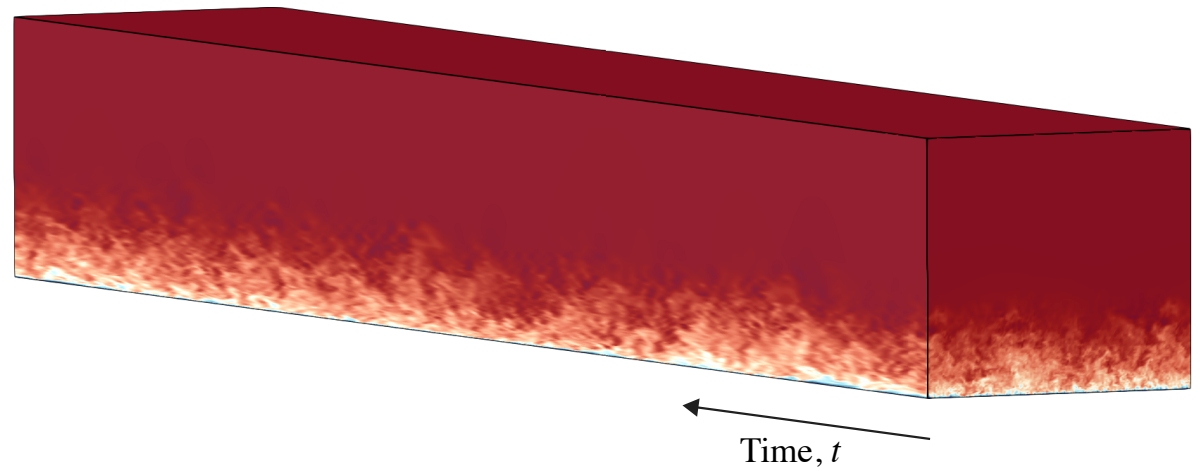

Fig. 3.3 TBL data generated for $R e_{\theta}=8000$, coloured by contours of velocity magnitude

In this work, boundary layer data is generated for $R e_{\theta}=8000$ and $R e_{\theta}=9800$. The data agrees well with reference LES data of a turbulent zero-pressure gradient boundary layer at $R e_{\theta}=8300$ from Eitel-Amor et al. [13] as shown in figure 3.4. The data is interpolated onto the inflow plane of the cases that are run, and is progressed through time into the computational domain. Note that since a finite amount of planes are collected, the data is restarted at the first plane after the last plane is reached. Figure 3.3 shows the turbulent boundary layer data box generated for $R e_{\theta}=8000$.

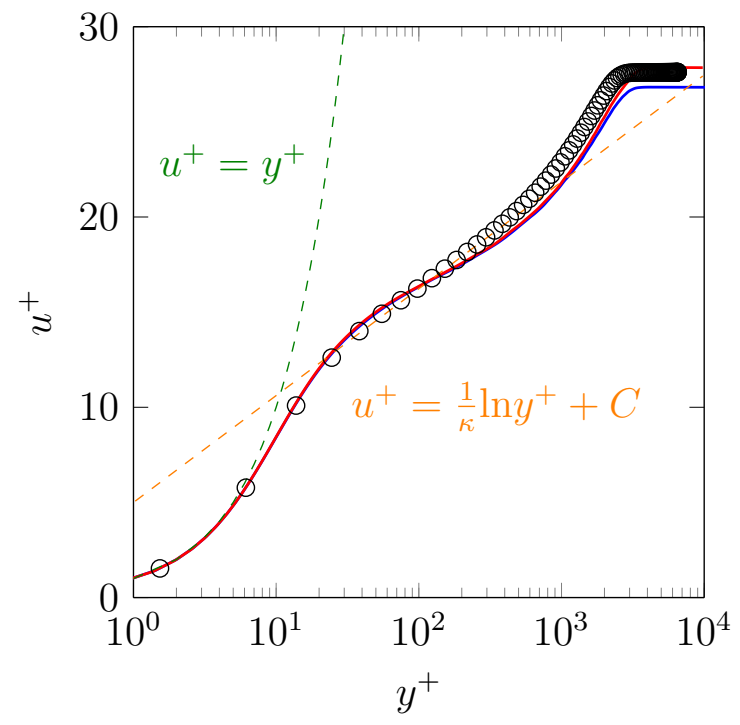

(a) Mean velocity profiles

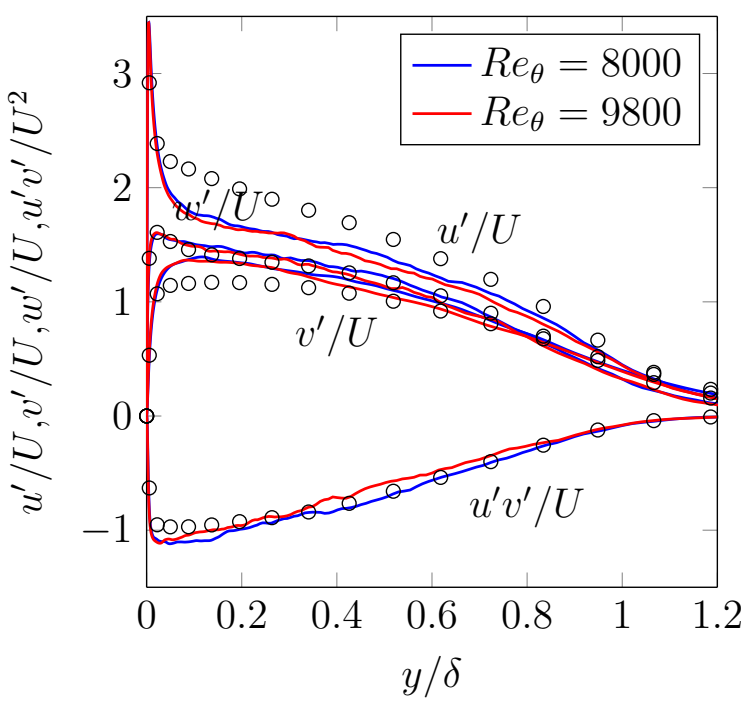

(b) Reynolds stress profiles

Fig. 3.4 Mean velocity and Reynolds stress profiles of the two TBLs generated using the Lund recycling method. Lines: Lund recycling TBL data, Symbols: reference LES data at $\operatorname{Re}_{\theta}=8300$ from Eitel-Amor et al. [13] 


\section{Chapter 4}

\section{Canonical Test Case 1: NASA Hump}

This project will first aim to validate the HYDRA CFD code and modifications described in chapter 3 against two simplified canonical test cases. These represent a breakdown of the intake flow physics. The first of these cases is a subsonic wall mounted hump case that features attached flow acceleration followed by downstream separation and reattachment. Recall that flow separation is important in engine intakes as it can lead to distortion at the fan face. Therefore it is important for predictive methods to reproduce this correctly.

RANS, LES and hybrid RANS-LES methods are investigated here. The acceleration over the curved hump forebody (although mild compared to intakes at incidence) exceeds the acceleration parameter threshold for which relaminarisation is suggested to occur. As such, the relaminarisation and SARC modifications to the SA model are investigated. Wall resolved LES is run to benchmark HYDRA's performance, and investigate the sensitivity of SGS model choice. Particular attention is paid to the size of the separation bubble. As previously discussed, RANS tends to under predict the turbulence in the separated shear layer, and therefore tends to predict too long a separation bubble. The results of the RANS and LES investigations are used to run a hybrid RANS-LES. Performance of these methods is discussed.

\subsection{Case Details}

The hump geometry is a Glauret-Goldschmied type body from Greenblatt et al. [14], herein referred to as the NASA hump, and featured as Case 3 at the NASA Langley Research Centre Workshop [73]. The experimentation was conducted in the open- 
return NASA Langley shear flow tunnel. A rich experimental data set can be found at http://cfdval2004.larc.nasa.gov/case3.html [74]. The hump model features a slot at approximately $65 \%$ chord near the point of separation for flow control investigation, although only the baseline uncontrolled case is considered in this work.

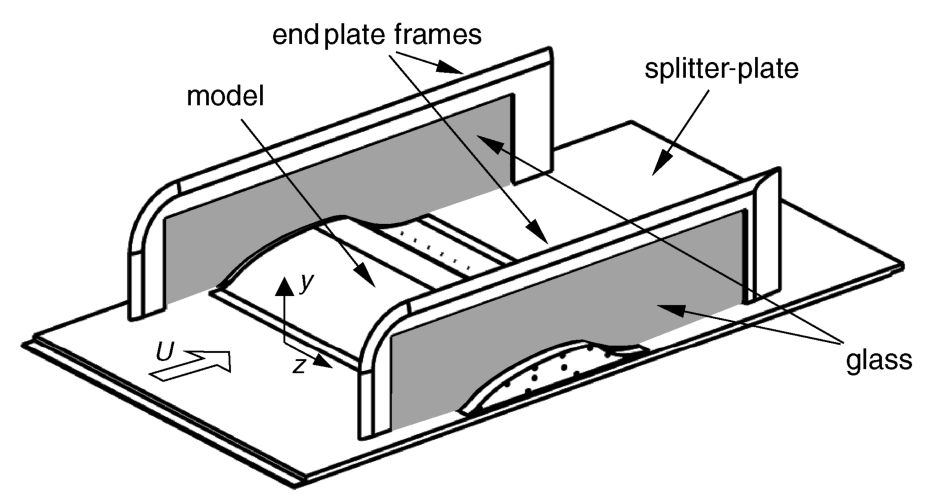

Fig. 4.1 NASA hump model showing the experimental features from [14]

Figure 4.1 shows a model of the experimental features of the NASA hump. The model has a relatively long convex forebody with a short ramp at the aft of the model. The chord length is $C=0.42 \mathrm{~m}$ with a maximum height of $0.128 C$ and a span of $1.390 \mathrm{C}$. The experimental setup features endplates at the extents of the hump span, giving a nominally two-dimensional flow, with three-dimensional sidewall effects limited to near the end plates. The model is mounted on a splitter plate, giving an effective wind tunnel height of $0.909 C$ and width $1.693 C$. A schematic of the hump is shown in fig. 4.2. Dimensions are normalised by hump chord length $C$.

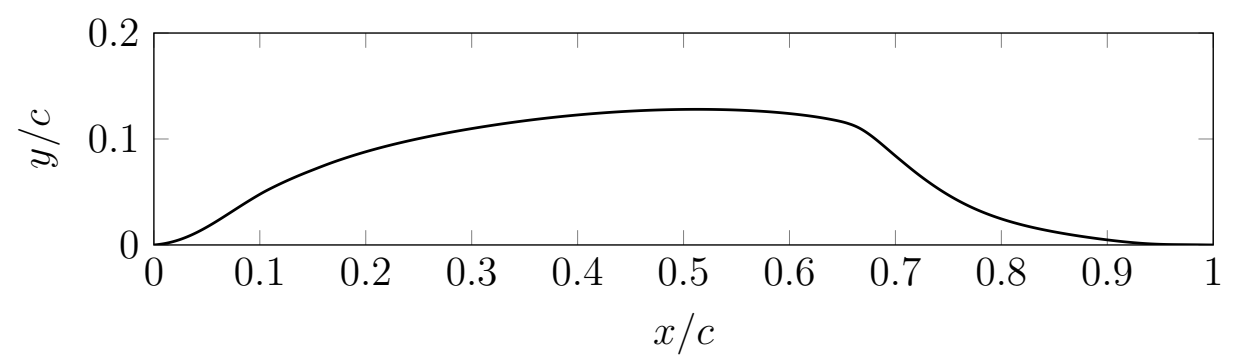

Fig. 4.2 Schematic of the NASA hump geometry normalised by the hump chord length $\mathrm{C}$

The reference freestream velocity for this case is $U_{\infty}=34.6 \mathrm{~m} / \mathrm{s}\left(M_{\infty}=0.1\right)$. This gives a Reynolds number based on the hump chord length of $R e_{C}=9.36 \times 10^{5}$. Flow approaching the hump first experiences an adverse pressure gradient at the leading foot of the hump, followed by a strong favourable pressure gradient accelerating the 
flow over the hump forebody. A second adverse pressure gradient towards the rear of the hump causes flow separation and subsequent reattachment and boundary layer recovery. The NASA hump gives peak acceleration parameter of approximately $K_{S}=$ $3.0 \times 10^{-6}$.

Experimental measurements of velocity and Reynolds stress profiles are taken at 6 locations downstream of flow separation, as well as one location upstream of the hump to characterise the oncoming boundary layer. In addition to this, a plane of velocity and turbulence data is measured along the tunnel center line, roughly covering the region $0.63<x / c<1.39$, to provide a cross section of the separation. Pressure coefficients $\left(C_{P}\right)$ and skin friction coefficient $\left(C_{f}\right)$ measurements are made along the hump surface.

\subsection{Computational Setup}

This work considers the two-dimensional hump flow without side plates. Following this, RANS computations are efficiently run using a 2D steady approach. For LES and hybrid RANS-LES, simulations are run 3D and unsteady using a shorter extent of the experimental domain in the spanwise and streamwise directions to reduce the computational cost. To enable this, turbulent boundary layers (TBLs) are used at the domain inlets.

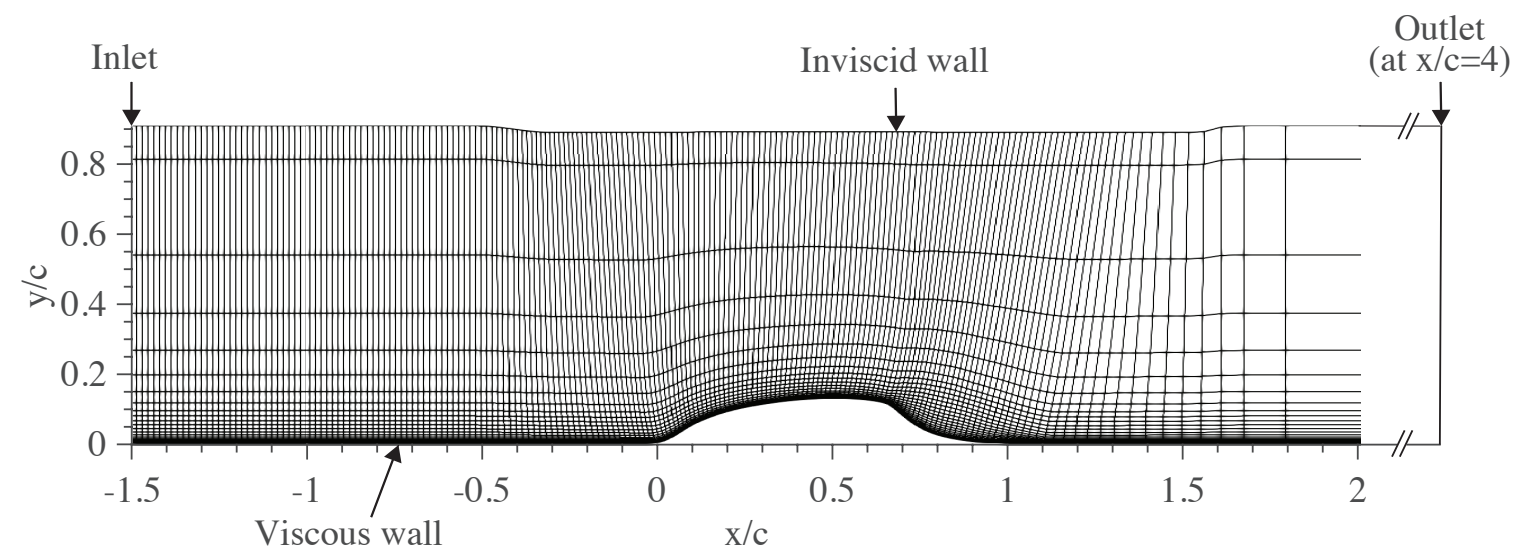

Fig. 4.3 Two-dimensional slice of the computational grid and boundary conditions used for LES of the NASA hump. Every 8th point shown in the streamwise direction and every 4th point shown in the wall normal direction for clarity. Note the contour on the top wall to account for endplate blockage.

Fully structured grids are used for the NASA hump simulations. A baseline grid designed to LES specifications is generated from which RANS and hybrid RANS-LES 
grids are derived. Figure 4.3 shows a two-dimensional slice of the computational grid used for LES. The grid extends $-1.5 C<x / c<4 C$ in the streamwise direction, with the hump located between $0<x / c<1$. The grid is extruded $0.2 C$ in the spanwise direction to form the $3 \mathrm{D}$ computational domain, which corresponds to about three inflow boundary layer heights. This domain width is chosen based on previous LES works of this case by numerous authors [75-77]. You et al. [78] showed that the pressure coefficients predicted with $L_{z}=0.15 C$ and $L_{z}=0.2 C$ had little difference [79]. Further, other authors such as Krishnan et al. [80] have successfully performed DES of the hump flow with a substantially smaller domain width $\left(L_{z}=0.121 C\right)$. As such, $0.2 C$ is considered suitable here. The wall normal domain size, $0.909 C$, is chosen to match the experimental wind tunnel height. Since the endplates present in the NASA hump experiment are not explictely modelled in this work, the effect of their blockage on the flow is accounted for by contouring the top wall of the computational domain between $-0.5<x / c<1.6$ as shown in figure 4.3. The shape of this is provided by the NASA Workshop [74] and has been successfully used in multiple CFD works $[75,76,78,79,81-83]$.

Grid points are clustered near the point of flow separation $(\approx 0.65 C)$ as well as upstream of the hump to resolve the inflow turbulent boundary layer. Downstream of the hump, the grid is gradually coarsened towards the outflow boundary to smooth flow fluctuations and prevent reflections at the boundary. The LES grid is developed to have a grid spacing of $\Delta x^{+}=90, \Delta y^{+}=1$ and $\Delta z^{+}=21$ based on the inflow boundary layer, where $\Delta x^{+}, \Delta y^{+}$and $\Delta z^{+}$are the grid spacings in the streamwise, wall normal and spanwise directions respectively. This is to ensure adequate LES resolution as recommended by Tucker [16] (see table 2.1). To achieve this, the LES grid has 1292 points in the streamwise direction, 110 points in the wall normal direction and 225 equally spaced points in the span. This gives the LES grid a total of 32 million points. The resolution is equal to or higher than previous LES works of this case [75-77], and as such a grid independence study is not run here. It should also be noted that as mesh resolution is increased for an LES, new modes continue to be resolved until DNS level is reached, and so the concept of a grid-independent solutions does not really exist for LES [75]. Furthermore, the aim of the present study is to compare the performance of various SGS models, which are all carried out under the same conditions.

For RANS simulations, a two-dimensional slice of the LES grid is used as the RANS computational domain. However, the upstream extent is increased from $-1.5 \mathrm{C}$ to $-6.39 C$ as recommended by Krishnan et al. [80] to allow the boundary layer to naturally develop to the profile measured by experiments at $x / c=-2.14$. Boundary 
layer velocity and turbulence intensity profiles are experimentally recorded here to validate the correct prescription of the oncoming boundary layer, with all models reproducing this well. Figure 5.5 shows the velocity profile of the boundary layer at $x / c=-2.14 C$ obtained from RANS-SA compared to experimental measurements.

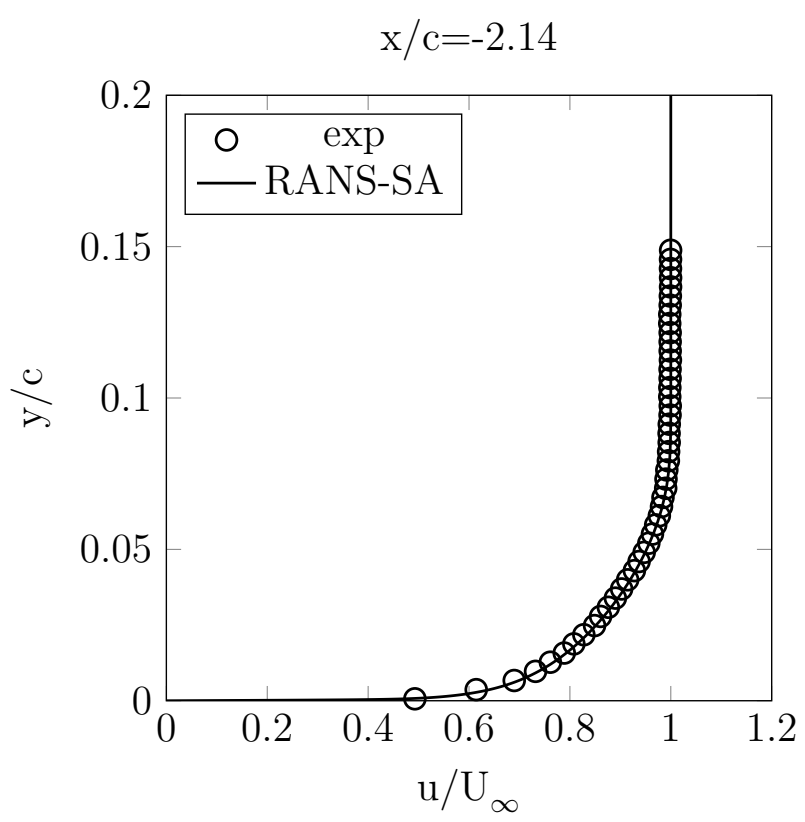

Fig. 4.4 Boundary layer velocity profile at $x / c=-2.14 C$ obtained from RANS-SA compared to experimental measurements.

For hybrid RANS-LES simulations, the same computational domain size as LES is used, however the baseline LES grid is coarsened by a factor of approximately 2 in the streamwise and spanwise directions. This gives a grid spacing $\Delta x^{+}=180, \Delta y^{+}=1$ and $\Delta z^{+}=42$, and is in the range recommended for hybrid RANS-LES by Tucker [16] (see table 2.1). The hybrid RANS-LES grid has 785 points in the streamwise direction, 110 points in the wall normal direction and 105 equally spaced points in the span. This gives the hybrid RANS-LES grid a total of 9 million points.

For all simulations, the boundary conditions used for the hump and wind tunnel floor are no-slip viscous walls, the top wall is modelled as an inviscid wall and the outflow is a fixed pressure outlet. Periodic boundary conditions are applied at the spanwise extents for LES and hybrid RANS-LES simulations. At the inlet, a uniform inflow boundary condition is applied for RANS simulations, with the freestream velocity $U_{\infty}=34.6 \mathrm{~m} / \mathrm{s}$. LES and hybrid RANS-LES simulations use an unsteady turbulent boundary layer of $R e_{\theta}=8000$ and thickness $\delta=0.026 \mathrm{~m}(0.062 C)$ at the inflow, generated using the method described in section 3.6. To obtain the $R e_{\theta}$ and $\delta$ needed here, 
the RANS solution is used since no experimental data is available at $x / c=-1.5 C$, only at $x / c=-2.14 C$. This is believed to be reliable since the RANS solution agrees very well with the measurements at $x / c=-2.14 C$, and is also expected to perform well in this flat attached part of the flow.

RANS simulations are run using the HYDRA steady flow solver with four levels of multi-grid. LES and hybrid RANS-LES simulation are run using the explicit unsteady solver, using converged RANS solutions to initialise the flow. The Smagorinsky-Lilly (SM), WALE and $\sigma$ SGS models are run for LES to investigate their performance for this flow. For all eddy resolving simulations, the flow is advanced $3 T^{*}$ before flow statistics are collected, where $T^{*}$ is the flow-through time, defined $T^{*}=C / U_{\infty}$. This allows the inflow TBL to develop, and flush initial transients from the region of interest. After this, time averaged flow statistics are collected over approximately $6 T^{*}-8 T^{*}$. For analysis, the solutions are further spatially averaged in the spanwise direction. This significantly reduces the time taken to acquire converged statistics.

\subsection{Results}

\subsubsection{RANS}

The results of RANS and RANS modifications are first investigated. Static pressure coefficient $\left(C_{P}\right)$ distributions computed over the NASA hump are compared with experimental measurements in fig. 4.5. Note that negative $C_{P}$ values have been plotted, which represent a decrease in pressure in the positive axis direction. $C_{P}$ values are computed as,

$$
C_{P}=\frac{\left(P-P_{\infty}\right)}{\frac{1}{2} \rho_{\infty} U_{\infty}^{2}}
$$

where $P$ is the local static pressure and $P_{\infty}, U_{\infty}$ and $\rho_{\infty}$ are reference values at the domain inlet.

$C_{P}$ meaurements show an adverse pressure gradient at the leading foot of the hump, followed by a favourable pressure gradient illustrated by a sharp increase in $-C_{P}$. This indicates the strong flow acceleration over the hump. This peaks at $x / c=0.52$ and returns to an adverse pressure gradient at the point of separation. Flow is experimentally measured to separate at $x / c=0.65$ and reattach at $x / c=1.11$, giving a bubble size of $x / c=0.46$. The NASA hump shows a small plateau in $C_{P}$ just downstream of $x / c=0.65$, which is a characteristic of separation [75]. The standard SA model performs well at predicting $C_{P}$ at the attached regions of the flow, i.e. upstream and 


- RANS-SA
$\cdots$ RANS-SA (Prod. Boost)

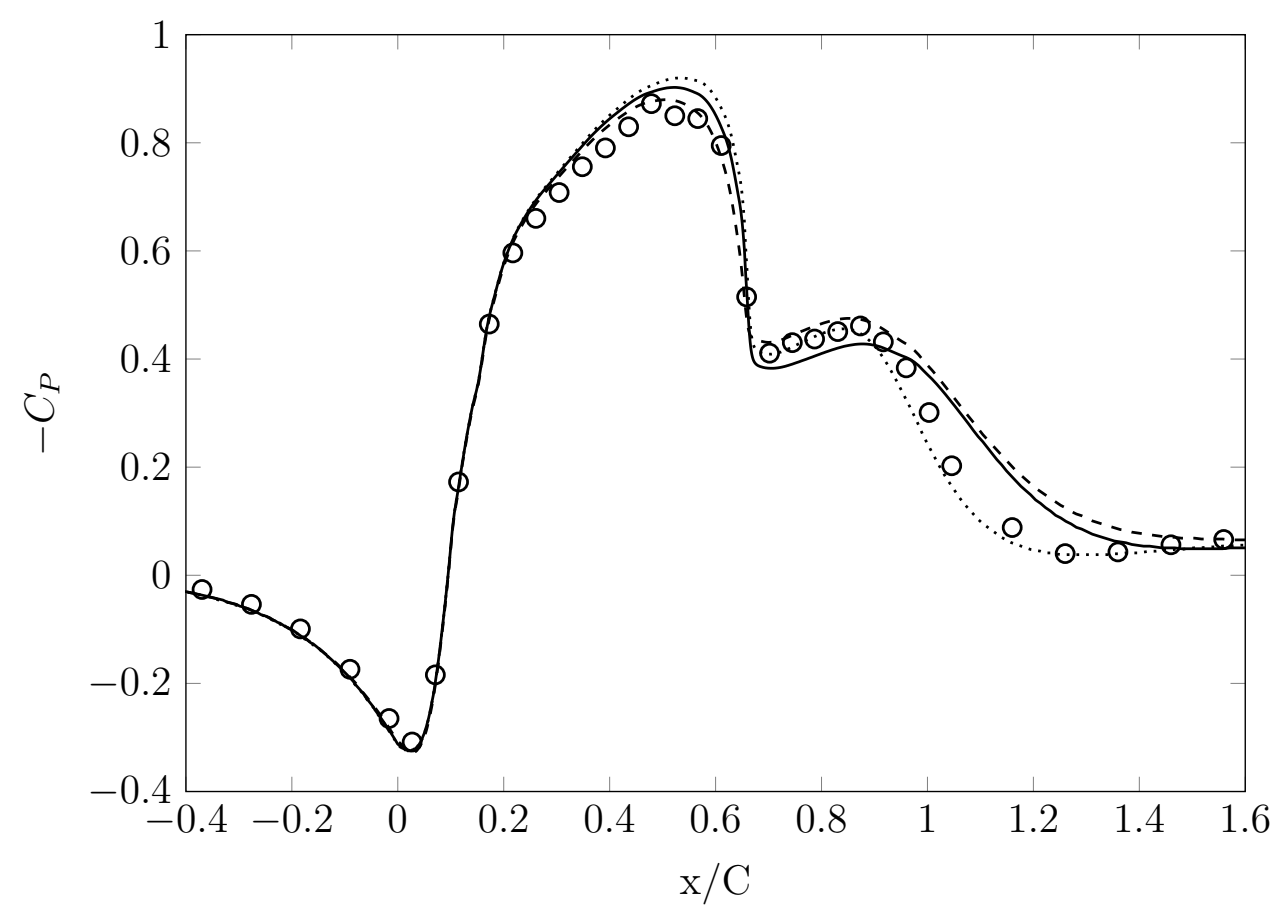

Fig. $4.5 C_{P}$ distribution over the NASA hump using RANS

over the hump forebody, as well as downstream of reattachment, as is expected. However, the model shows an over prediction of peak $-C_{P}$ by approximately $7 \%$ as well as an under prediction of the pressure plateau. Downstream of the separation point, we also see the standard model unable to sufficiently capture the post separation pressure recovery. This is an indication of an over-predicted bubble size and delayed flow reattachment. The standard RANS-SA model is able to predict the separation point correctly, but reattaches late at $x / c=1.67$. This is an over-prediction of the bubble size by $46 \%$. Note that the separated region is defined where the axial wall shear stress $\tau_{w}<0$.

The modified RANS-SA model for relaminarisation and curvature (labelled RANSSA Mod) shows an improvement in the peak $-C_{P}$ prediction compared to the standard SA model. This is brought down in agreement with the experimental measurements. The model also correctly predicts the pressure plateau, improving over the standard model. Downstream of separation however, the modified RANS-SA model returns back to standard RANS-SA behaviour as expected, predicting flow reattachment much later than experiment. 
Figure 4.6 shows the acceleration parameter $K_{S}$ distribution over the NASA hump forebody. As shown, $K_{S}$ peaks to $K_{S}=3 \times 10^{-6}$ (exceeding the threshold of laminarisation described by Loyd et al. [28] and Launder and Stinchombe [29]) although this is over a relatively short streamwise extent. This shows that, although subtle, sensitivity to relaminarisation and curvature can improve mean surface pressure predictions even for mild accelerations.

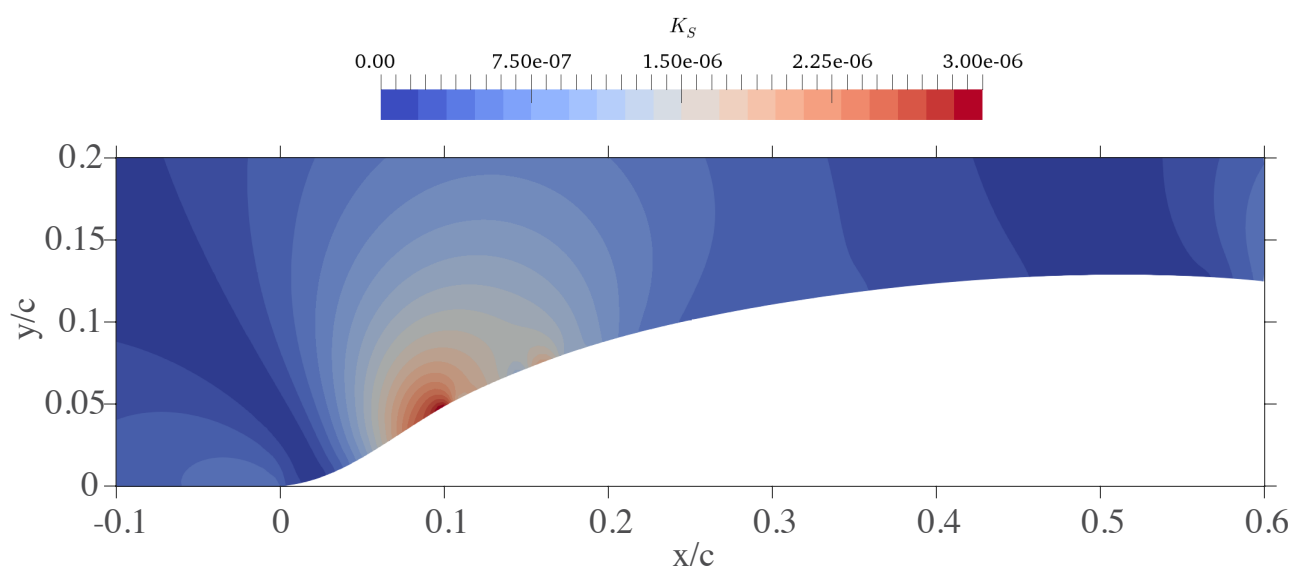

Fig. $4.6 K_{S}$ distribution over the NASA hump forebody

As mentioned, RANS models over predict separation size due to insufficient turbulence production within the separated region. To quantify this, turbulence production is manually boosted post separation over the hump until the correct reattachment location is achieved. This is done by multiplying the RANS-SA model's production term $P(\tilde{\nu})$ in equation (3.14) by a simple linear ramp function $\gamma_{P}$ to increase production over the length of the separation.

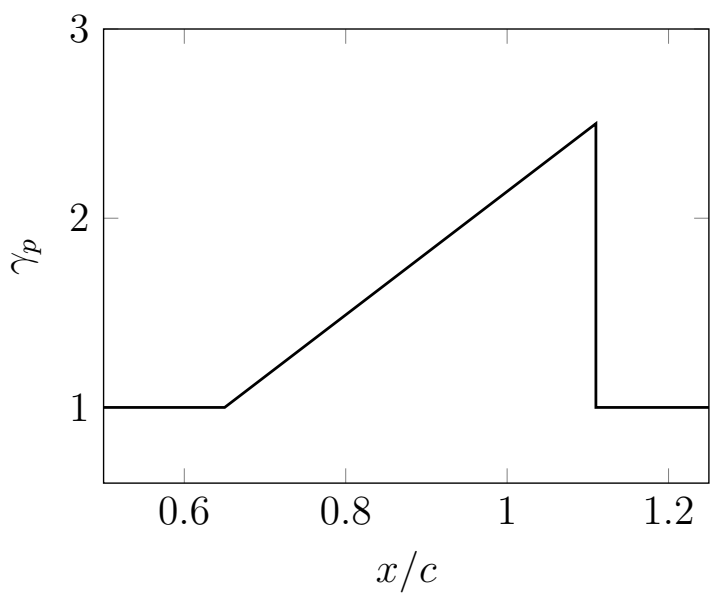

Fig. 4.7 Linear ramp function used to boost production in the separated region 


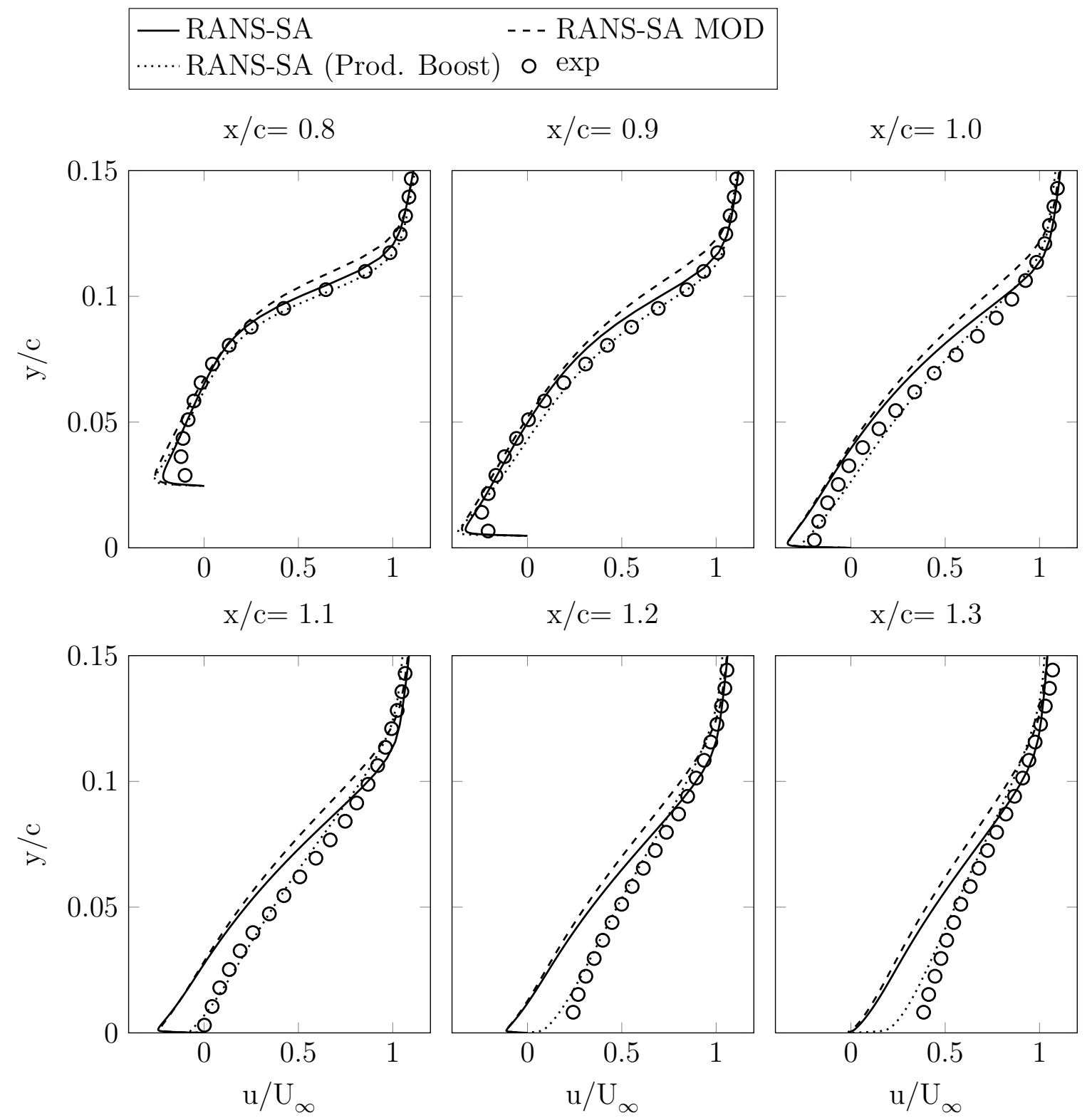

Fig. 4.8 Streamwise velocity profiles located downstream of the separation compared with experimental measurements for RANS

Figure 4.7 shows the variation of $\gamma_{p}$ in the separated region. It is found that a production ramp up to 2.5 times the standard RANS-SA production is needed to correctly reattach the flow. The resulting $C_{P}$ distribution (labelled RANS-SA (Prod. Boost)) in figure 4.8 then agrees very well with experimental measurements. Of course this manual boosting of turbulence is carried out only to display the extent of deficiencies of standard RANS in separated flow regions, and would not be possible without correct prior knowledge of the flow. 
Streamwise velocity profiles located downstream of the separation point are compared with experimental measurements in figure 4.8. Velocities are non-dimensionalised with the freestream velocity $U_{\infty}$. Six measurement locations are compared, located at $x / c=0.8,0.9,1.0,1.1,1.2$ and 1.3 . The standard RANS-SA model initially performs well post separation, but deviates from experimental measurements further downstream into the separated zone. The model continues to show flow reversal far downstream compared to experimental measurements, highlighted at $x / c=1.1$. This again displays its over prediction of the separation size. As with pressure predictions, the modified RANS-SA model has little effect here, behaving very similar to standard RANS-SA. It is only by manually boosting turbulence production in the separated flow that agreement with experimental measurements can be achieved.

\subsubsection{LES and Hybrid RANS-LES}

Figure 4.9 shows instantaneous Q-criterion contours from LES over the NASA hump using the $\sigma$-SGS model. Q-criterion is used to visualise vortical structures in the flow and is previously defined in equation (2.5). The figure shows the oncoming inflow turbulent boundary layer used for LES and hybrid RANS-LES as it is convected over the hump body, as well as turbulent eddies in the reversed flow (separated) region.

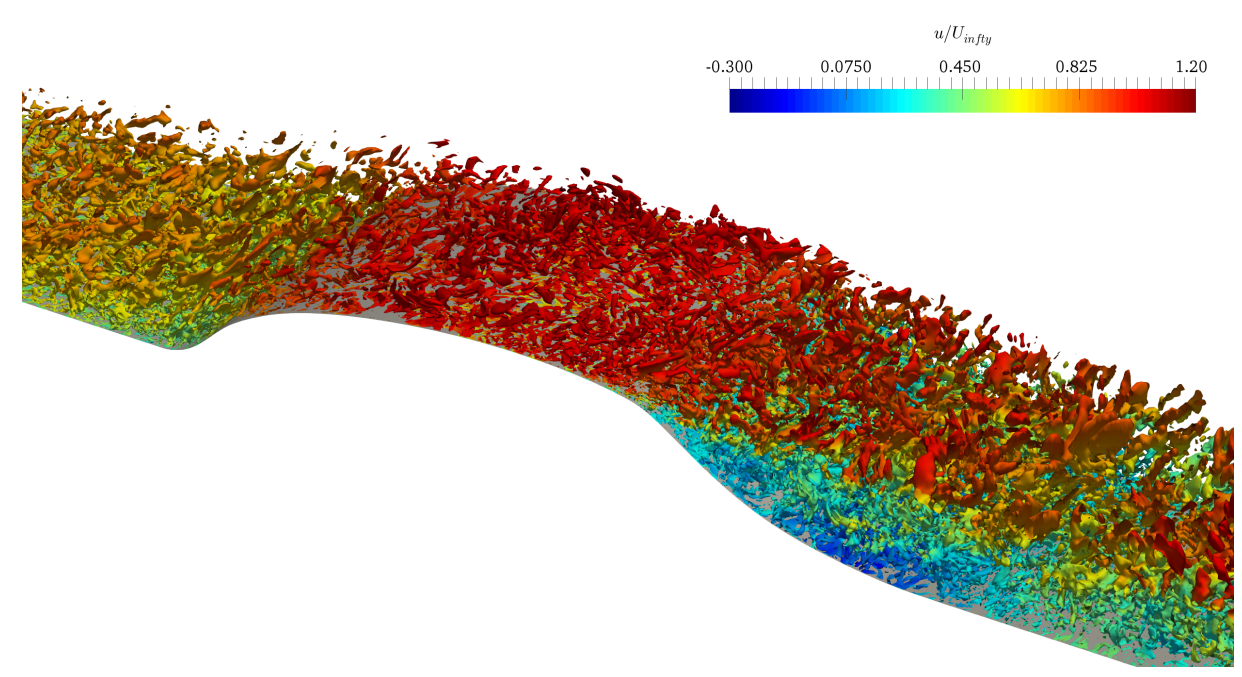

Fig. 4.9 Instantaneous Q-criterion contours coloured by velocity over the NASA hump using LES with the $\sigma$-SGS model. The flow direction is left to right.

Time averaged $C_{P}$ distributions over the hump using the LES-SM, WALE and $\sigma$-SGS models are shown in figure 4.10. It is immediately clear that the SM-SGS model performs poorly for this flow, with peak $-C_{P}$ severely under-predicted, as 
well as poor pressure recovery following separation. This is significantly worse than even the standard RANS-SA model prediction. The WALE and $\sigma$-SGS models on the other hand agree very well with experimental surface pressure measurements. These improve on peak $-C_{P}$ prediction where the standard RANS-SA shows an overprediction, and also capture the separation plateau and $C_{P}$ in the recirculation region well. We can also note the close performance of the WALE and $\sigma$-SGS models despite their significantly different formulations. This is encouraging and shows eddy resolving methods' superiority at predicting separated flows naturally without modification.

\begin{tabular}{ll|}
\hline - RANS-SA & LES-SMAG \\
- - LES-WALE & LES- $\sigma$ \\
- Hybrid RANS-LES & $\circ$ exp \\
\hline
\end{tabular}

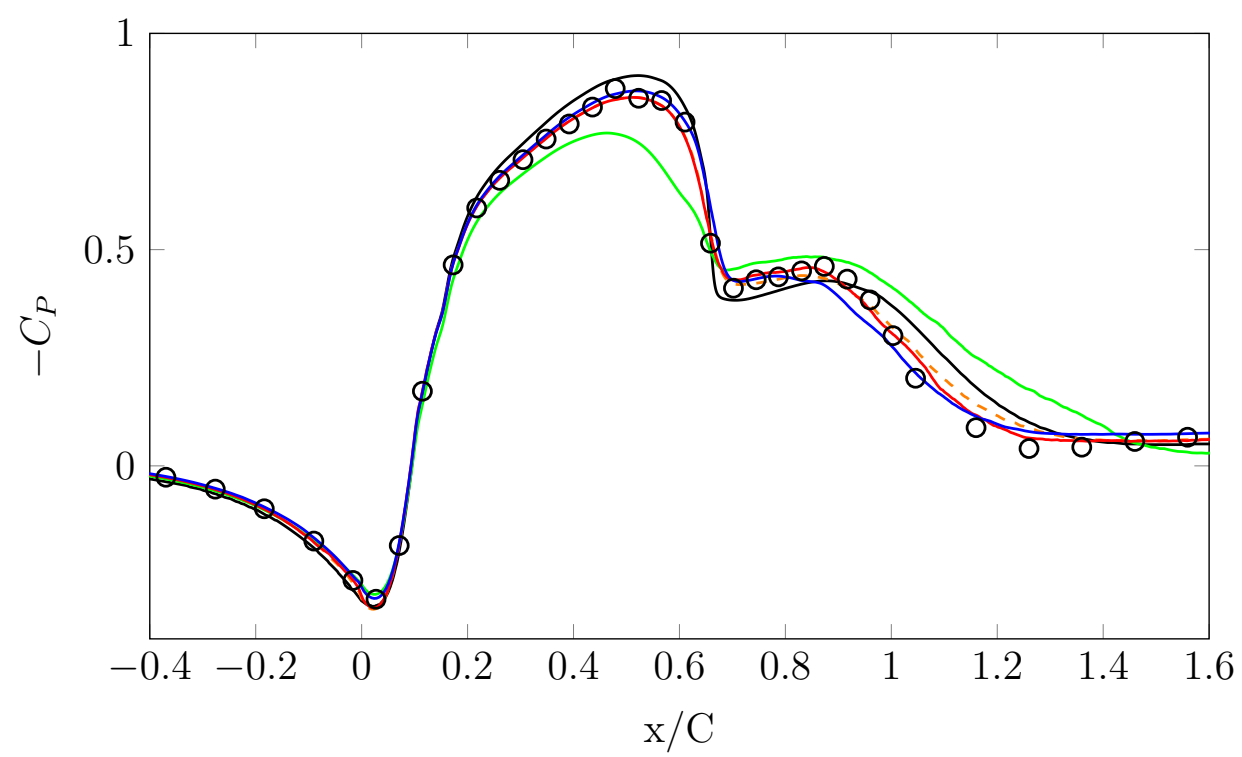

Fig. $4.10 C_{P}$ distribution over the NASA hump

Table 4.1 shows the separation, reattachment and bubble sizes predicted by simulations. Flow reattachment is largely governed by the turbulent kinetic energy production in the separation that drives the flow to reattach. Schemes that are too dissipative or lack sufficient turbulent production are unable to resolve finer high energy turbulence scales and as a result, over predict the separation size. From table 4.1, we see that all simulations are able to predict the separation point correctly except the LES-SM, which separates early. This is one of the causes for discrepancies with the LES-SM's $C_{P}$ distributions seen in figure 4.10. Figure 4.11 shows time averaged axial velocity contours downstream of separation compared to a plane of velocity measured experimentally along the NASA hump center line. This displays a cross section of the 
separated region. Flow reattachment locations are marked $x_{r}$ on figure 4.11, as well as a vertical dashed line showing the experimental reattachment point. Since the SM model separates early, the flow trajectory at the point of separation is less inclined towards the tunnel floor, and this may be a contributor to its delayed flow reattachment. This occurs at $x / c=1.33$ and matches that of RANS-SA, thus also over-predicting the separation by $44 \%$. We can see from figure 4.11 that LES-SM recirculation region is longer and has a thicker bubble than experimental measurements. The LES-WALE and LES- $\sigma$ models perform much closer to experiments here, reattaching at $x / c=1.19$ and $x / c=1.15$ respectively. While these are still unable to match experiment (overpredicting the bubble size by $17 \%$ and $8 \%$ respectively), these are much improved over RANS and LES-SM, with the $\sigma$-SGS model performing best. The separation bubble shape and size also qualitatively agree well with experiments as shown in figure 4.11.

\begin{tabular}{|l|l|l|l|}
\hline & $\begin{array}{l}\text { Separation } \\
\text { Point }(\mathrm{x} / \mathrm{c})\end{array}$ & $\begin{array}{l}\text { Reattachment } \\
\text { point }(\mathrm{x} / \mathrm{c})\end{array}$ & $\begin{array}{l}\text { Bubble } \\
\text { size }(\mathrm{x} / \mathrm{c})\end{array}$ \\
\hline Exp & 0.65 & 1.11 & 0.46 \\
\hline RANS-SA & 0.65 & 1.32 & 0.67 \\
\hline RANS-SA Mod & 0.65 & 1.33 & 0.68 \\
\hline LES-SM & 0.60 & 1.33 & 0.72 \\
\hline LES-WALE & 0.65 & 1.19 & 0.54 \\
\hline LES- $\sigma$ & 0.65 & 1.15 & 0.50 \\
\hline Hybrid RANS-LES & 0.65 & 1.17 & 0.52 \\
\hline
\end{tabular}

Table 4.1 Separation, reattachment and bubble size over the NASA hump

From these results, hybrid RANS-LES is run for the NASA hump using the $\sigma$-SGS model in the LES parts of the flow, and the modified RANS-SA model with relaminarisation and curvature corrections near the wall. This performs very close to LES- $\sigma$ for $C_{p}$ predictions as shown in figure 4.10, agreeing well with experimental measurements. Hybrid RANS-LES also performs well at predicting the flow separation as shown in figure 4.11, giving a reattachment location of $x / c=1.17$. This is within $4 \%$ of LES- $\sigma$ 's bubble size, despite the hybrid simulation costing less than $20 \%$ in computational cost compared to wall resolved LES. This suggests that the high grid resolution required by LES to resolve fine near turbulence is not needed in the separated flow region, where predominantly large scale eddies dominate. The reduced grid resolution used for hybrid RANS-LES is sufficient to resolve these and reattach the flow, displaying hybrid RANS-LES's suitability to separated flows. 

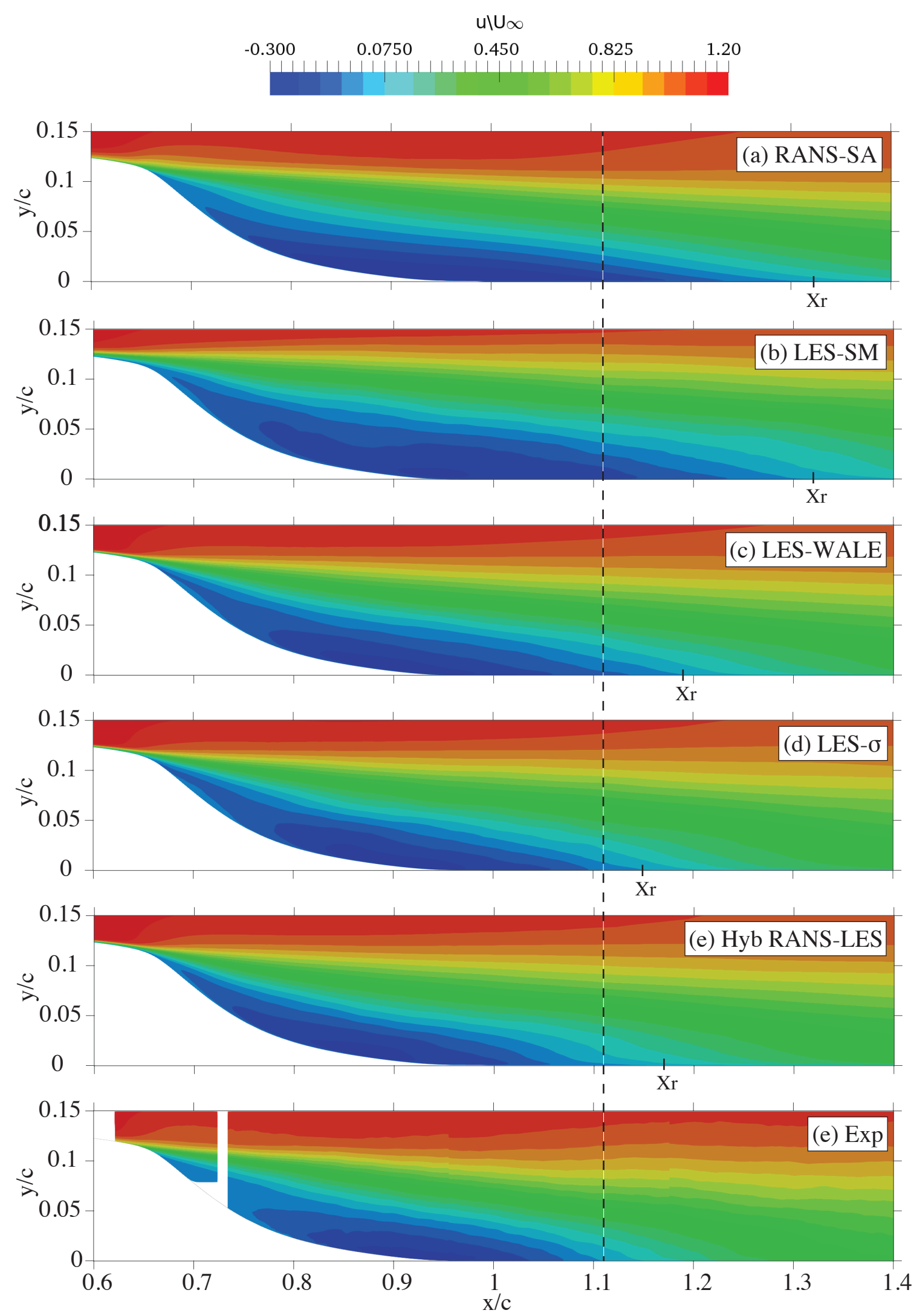

Fig. 4.11 Time averaged axial velocity contours downstream of separation from CFD compared to a plane. Flow reattachment locations are marked $x_{r}$. The vertical dashed line shows the experimental reattachment point 


\begin{tabular}{ll}
\hline - RANS-SA & LES-SM \\
- - LES-WALE & LES- $\sigma$ \\
- Hybrid RANS-LES & o
\end{tabular}
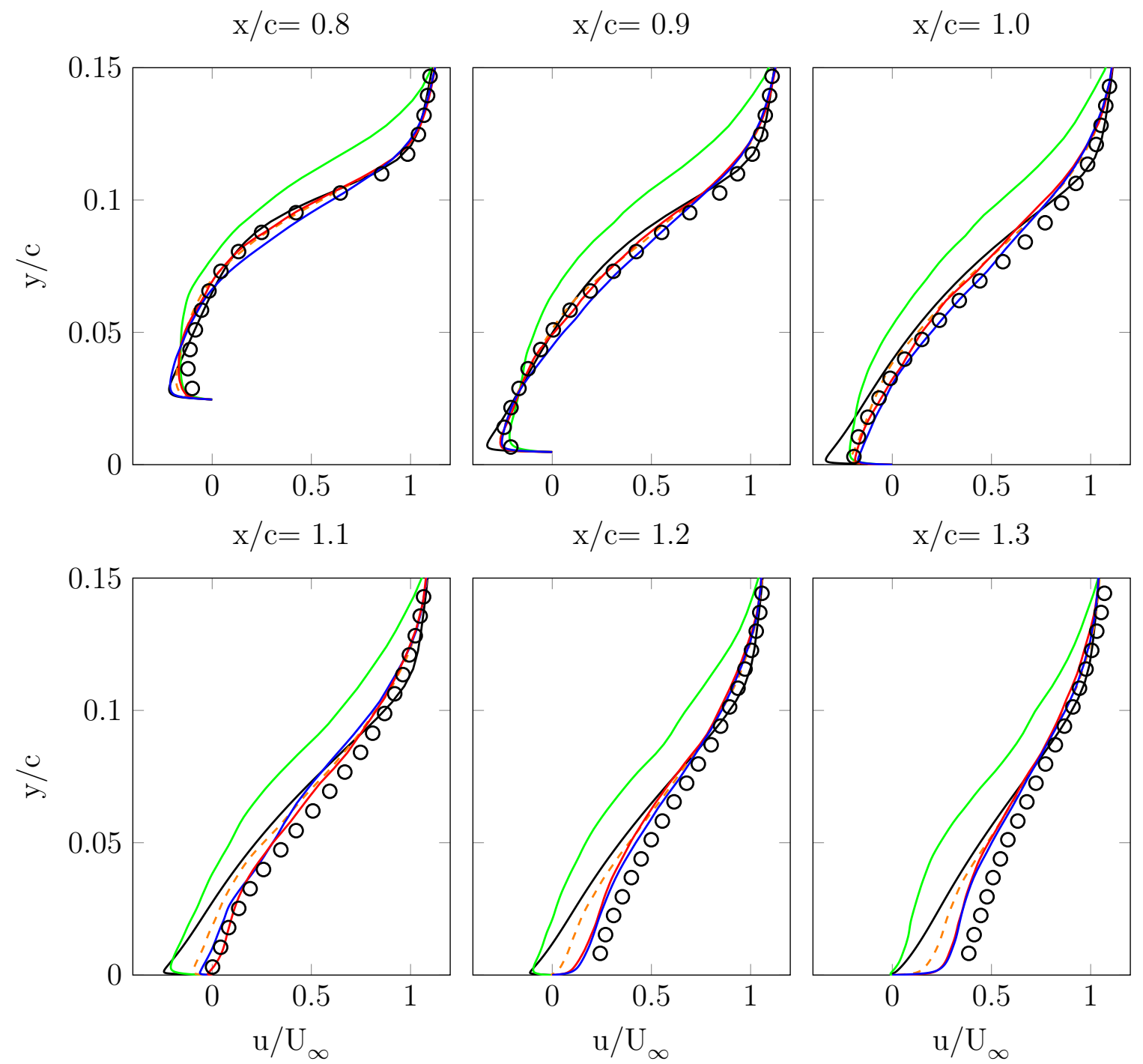

Fig. 4.12 Streamwise velocity profiles located downstream of the separation compared with experimental measurements for LES and hybrid RANS-LES 

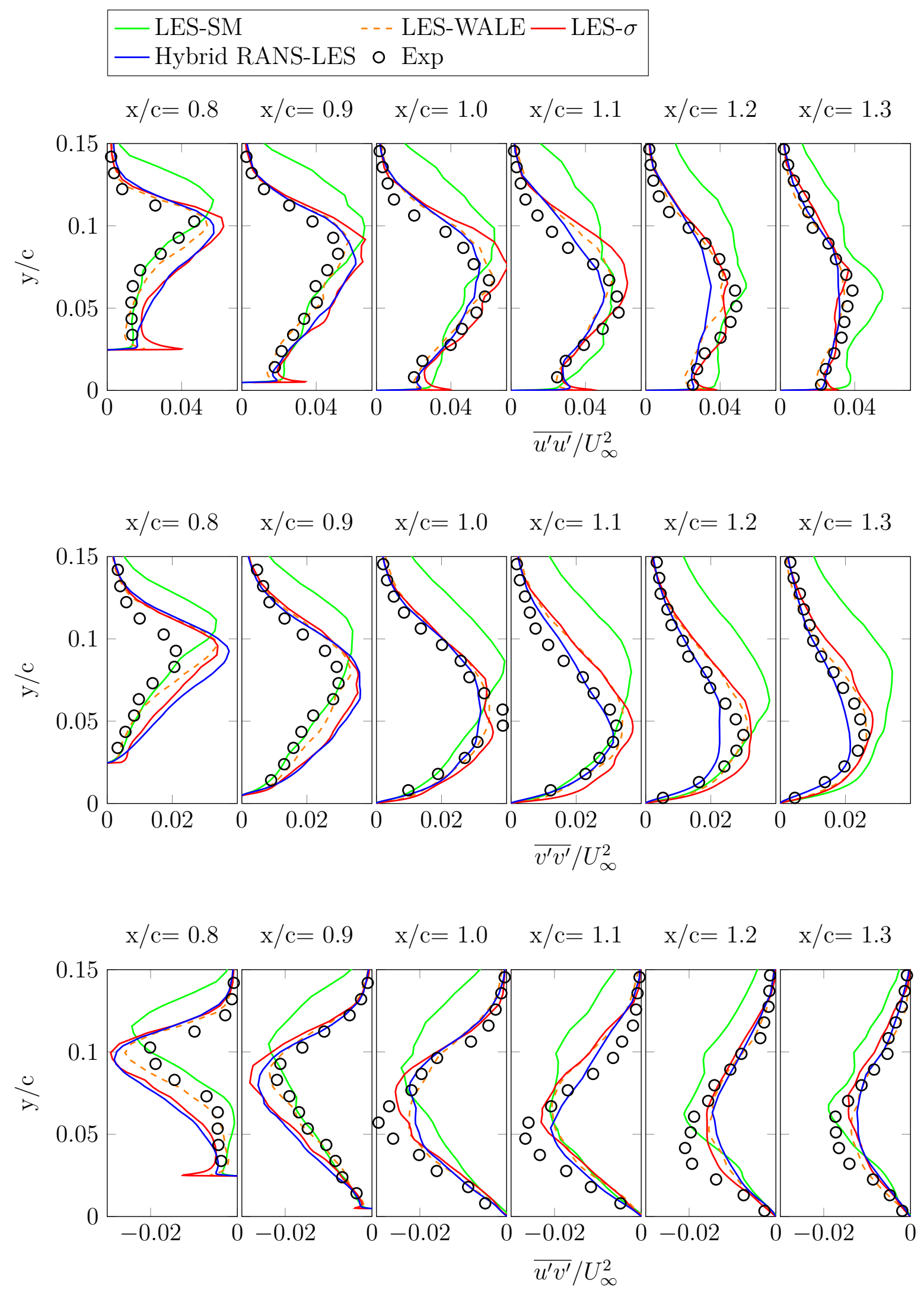

Fig. 4.13 Reynolds stress profiles located downstream of the separation compared with experimental measurements for LES and hybrid RANS-LES 
Figure 4.12 shows mean streamwise velocity profiles computed using LES and hybrid RANS-LES compared to experimental measurements. Here again we can see LES-SM's poor agreement with experimental measurements, whereby the profile shape is not predicted well. These show a thicker reversed flow area with a velocity deficit extending further into the freestream. This is in line with what is qualitatively observed in figure 4.11 (a greater separation bubble height) and could be attributed to premature separation. The remaining simulation methods are all able to predict the velocity profile shapes well, with LES- $\sigma$ performing best at all locations.

Since eddy resolving methods directly resolve turbulent fluctuations in space and time, these enable much more information to be extracted from simulation compared to RANS. Figure 4.13 shows predictions of $\overline{u u} / U_{\infty}^{2}, \overline{v v} / U_{\infty}^{2}$ and $\overline{u v} / U_{\infty}^{2}$ Reynolds stresses from the LES and hybrid RANS-LES simulation compared to experimental measurements. The locations correspond to those where velocity profiles are measured. It should be noted that experimental errors for turbulence measurements are estimated at $14 \%$ for $\overline{u u} / U_{\infty}^{2}$ and $\overline{v v} / U_{\infty}^{2}$ stresses, and as much as $20 \%$ for $\overline{u v} / U_{\infty}^{2}$. Model comparisons with this data should therefore be considered in a semi-quantitative manner.

The LES-WALE, LES- $\sigma$ and hybrid RANS-LES simulations are able to predict all three stress profiles shapes well compared to experiments. All stresses generally show good profile shape and magnitude predictions. However, peak stresses for all three components are over-predicted at the first measurement location $(x / c=0.8)$ by all simulations. For the $\overline{v v} / U_{\infty}^{2}$ profile at $x / c=0.8$ for example, the peak stress magnitude is over-predicted by approximately $50 \%$ by the LES-WALE and LES- $\sigma$ models. This is significantly higher than the experimental error range. For the remaining locations however, the peaks are predicted very well by LES-WALE and LES- $\sigma$. While hybrid RANS-LES does also perform well here, we can note that turbulent stress values are generally predicted less than those by LES- $\sigma$, which can be attributed to the coarser grid used here.

As with velocity profile predictions, the LES-SM simulations give incorrect prediction of the turbulent stress profile shape since the separation bubble is thicker, therefore skewing the profiles away from the wall. Despite this, the peak stress values do compare well with the other models.

One reason why the SM-SGS models performs poorly for this flow (separating prematurely and reattaching too late) could be that the model returns too high subgrid scale viscosity $\nu_{\text {sgs }}$ that is damping resolved turbulent fluctuations. However, as shown in figure 4.13, the resolved Reynolds stresses magnitude within the separation agree well with experiments and other LES SGS models which suggests this is not 
the case. Figure 4.14 shows time averaged contours of resolved turbulent kinetic energy (TKE) over the NASA hump for the three LES SGS models tested. These qualitatively compare well, with TKE within the separation of similar magnitude between the models.
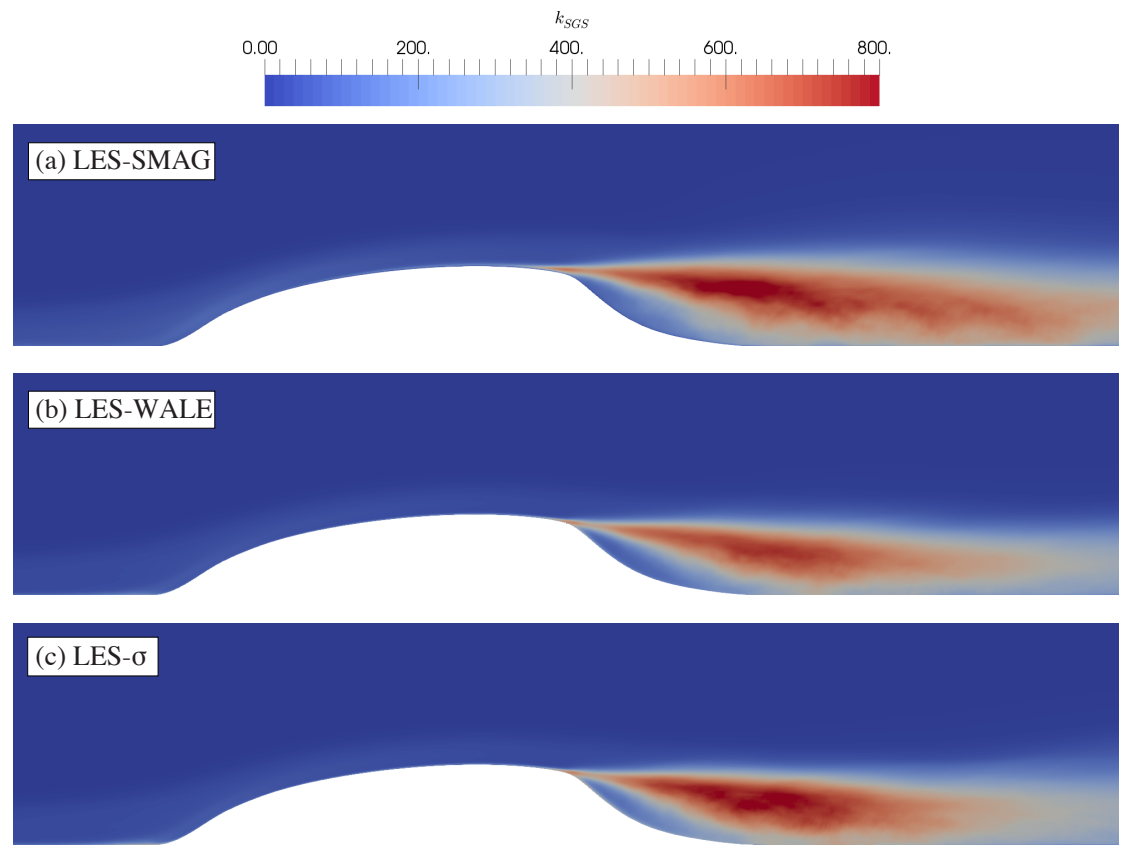

Fig. 4.14 Time averaged contours of resolved turbulent kinetic energy (TKE) over the NASA hump for the three LES SGS models tested

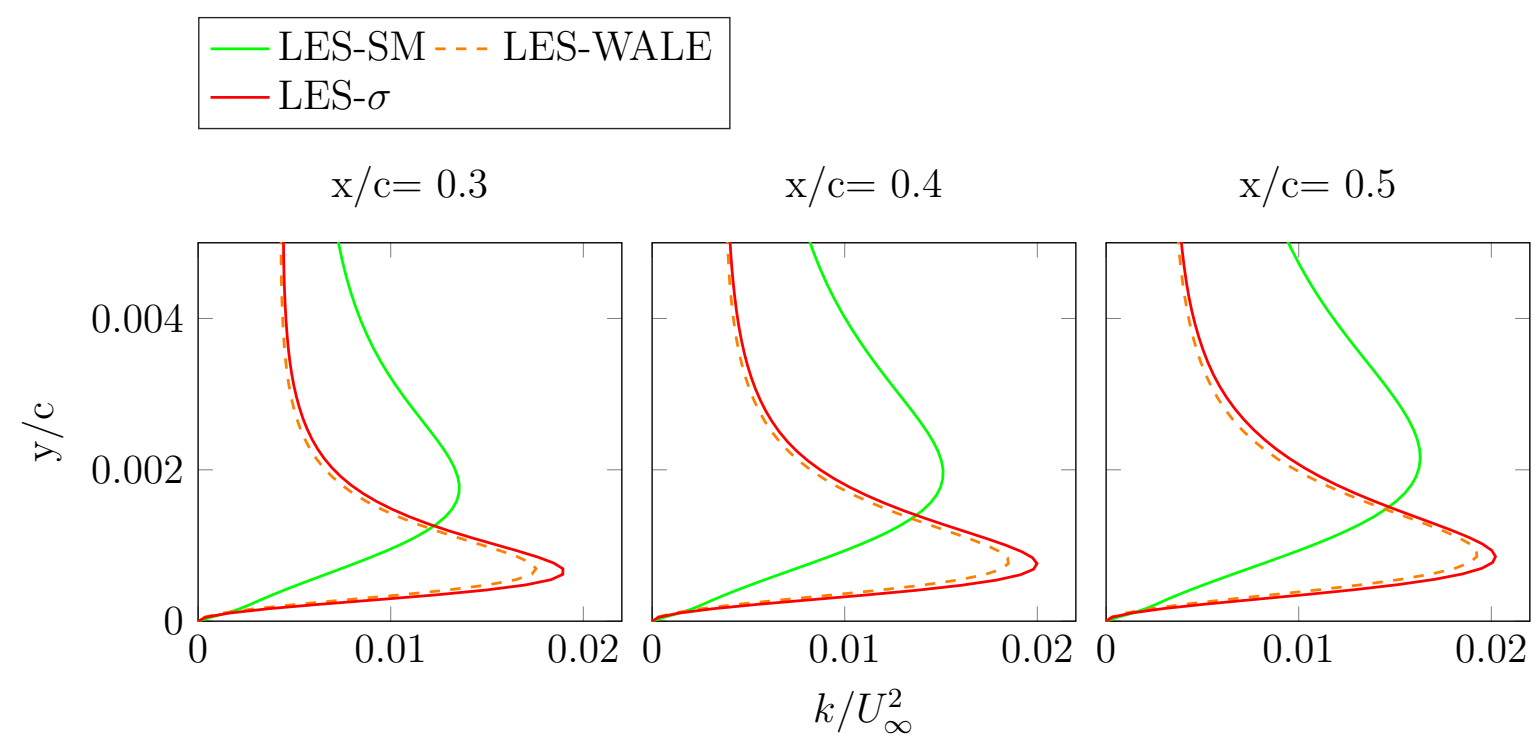

Fig. 4.15 Turbulent kinetic energy profiles upstream of separation on the hump surface for LES 
Investigation of the flow upstream of separation reveals that it may be the model's turbulence behaviour near the wall that causes LES-SM to separate early. Figure 4.15 shows TKE profiles near the wall at 3 locations upstream of separation on the hump surface. Profiles show that LES-WALE and LES- $\sigma$ models produce a higher peak TKE which is also concentrated closer to the wall compared to LES-SM. LES-SM only gives peak TKE of approximately $75 \%$ of the other models, with this distributed further away from the wall. This can be attributed to LES-SM's known poor performance near the wall, where wall damping is used, while the LES-WALE and LES- $\sigma$ are able to model this naturally (as discussed in section 3.3.1).

\subsection{Conclusions}

Relaminarisation and curvature corrections to the RANS-SA turbulence model show a noticeable improvement in surface pressure predictions for flow over a wall mounted hump model. Although mild, the acceleration over the hump body exceeds the threshold for which relaminarisation is suggested to occur. However, RANS fails to predict the post separation flow well, significantly over predicting the size of the separation bubble. Boosting the turbulence production by 2.5 times towards the end of the bubble is required to achieve the correct reattachment, and so RANS models must be used with caution when dealing with such flows.

LES is able to predict flow over the hump well, including acceleration over the hump and downstream separation, as long as the SM-SGS model is not used. This predicts premature boundary layer separation and delayed flow reattachment believed to be caused by its near wall turbulence behaviour upstream of separation. Flow is less sensitive to the WALE and $\sigma$-SGS models despite their significantly different formulations, suggesting they are well suited at reproducing the flow physics. These agree well with experimental data of velocity and Reynolds stress profiles within the separation, with the $\sigma$-SGS predicting a bubble size within $8 \%$ of measurements.

The $\sigma$-SGS is used to run a hybrid RANS-LES simulation of the flow coupled with the modified RANS model near the wall. This performs very close to LES- $\sigma$ despite costing less than $20 \%$ in computational cost. This is encouraging for simulations of aero-engine intake flow, where separation size, shape and reattachment locations are particularly important. 


\section{Chapter 5}

\section{Canonical Test Case 2: Axisymmetric Hump}

The second canonical test case in this work is an axisymmetric transonic hump model. This case follows naturally from the NASA hump and introduces transonic flow features. The case features smooth localised acceleration to supersonic flow over the hump body, followed by a near normal shock wave and SWBLI. This is a strong turbulent interaction that leads to flow separation and subsequent flow reattachment downstream. As with the NASA hump, the case aims to breaks down flow features of an intakes at incidence. This enables validation of the HYDRA CFD code and modifications against a simplified test case, before simulating the intake rig that unifies these complex flow physics.

This chapter will focus on the prediction of the shock location and nature of the SWBLI. These are fundamental flow features of intakes at incidence, and their correct reproduction is critical for predictive methods. Parameters such as the shock location can have a significant impact on the flow propagating towards the engine fan face. The behaviour of turbulence through this zone plays a critical role.

RANS and hybrid RANS-LES methods are investigated here. Wall resolved LES is prohibitively expensive for this case, which has a unit Reynold number of $R e=$ $13.6 \times 10^{6} / \mathrm{m}$, and is therefore not run. This chapter will focus on the hybrid RANSLES method's performance for this flow. As found in the previous chapter, the $\sigma$-SGS performs best for flow over the NASA hump, and so it is used for hybrid RANS-LES here. The Ducros sensor is also used and its ability at capturing shocks is compared to the Jameson sensor and how this affects the numerical smoothing field. Unlike the NASA hump, strong viscous-inviscid interactions caused by the shock and SWBLI are important here and their effect on the near wall turbulence will be explored. 


\subsection{Case Details}

The axisymmetric transonic hump model used in this work is from Bachalo and Johnson [44], who developed the model for the purpose of providing a validation case for turbulence models. The model consists of an annular circular-arc bump affixed to a circular cylinder aligned with the flow direction. The cylinder is mounted in the centre of the NASA Ames Research Center 2x2ft Transonic Wind Tunnel where experiments are conducted. The setup provides a high quality flowfield free from wall interference, three-dimensional effects, and excessive unsteadiness. This allows some of the fundamental features of the flow to be isolated and measure in detail.

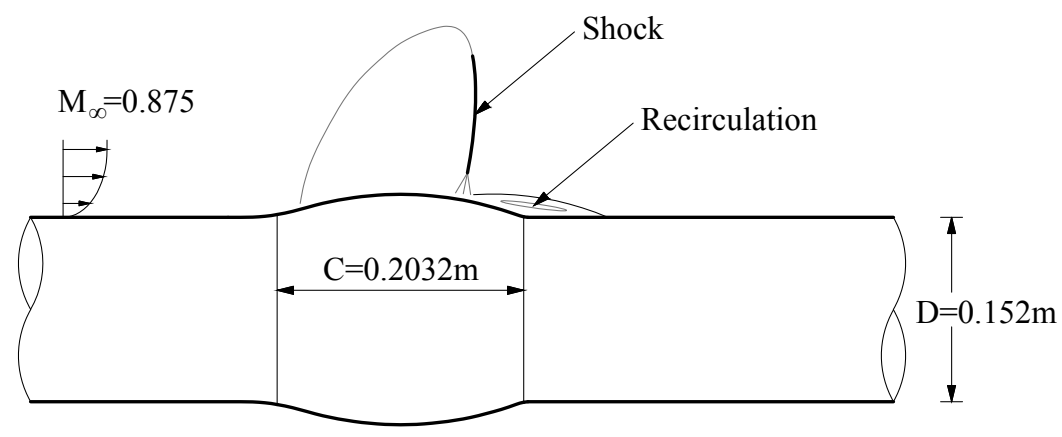

Fig. 5.1 Axisymmetric hump model showing the main flow features

Figure 5.1 shows the hump model and the main flow features. The hump has a chord length of $C=0.2032 \mathrm{~m}$ and the cylinder has an outside diameter of $D=0.152 \mathrm{~m}$. The freestream velocity for this case is $M_{\infty}=0.875$, which gives a Reynolds number based on the chord of $R e_{C}=2.8 \times 10^{6}$. The cylinder extends $3 C$ upstream of the humps leading edge. This allows natural transition of the flow to a fully developed turbulent boundary layer at the hump. Flow accelerates over the hump forebody to supersonic speed, forming a supersonic flow region. This terminates with a shock wave and SWBLI on the hump surface. The adverse pressure gradient imposed on the boundary layer here, as well as the trailing edge gradient of the hump surface, causes flow separation. A schematic of the hump geometry is shown in figure 5.2.

As with the NASA hump, experimental measurements of the velocity and Reynolds stress profiles are taken at 6 locations downstream of the shock and separation, as well as one location upstream of the hump to characterise the oncoming boundary layer. In addition to this, surface pressure measurements are made on the second half of the hump surface. 


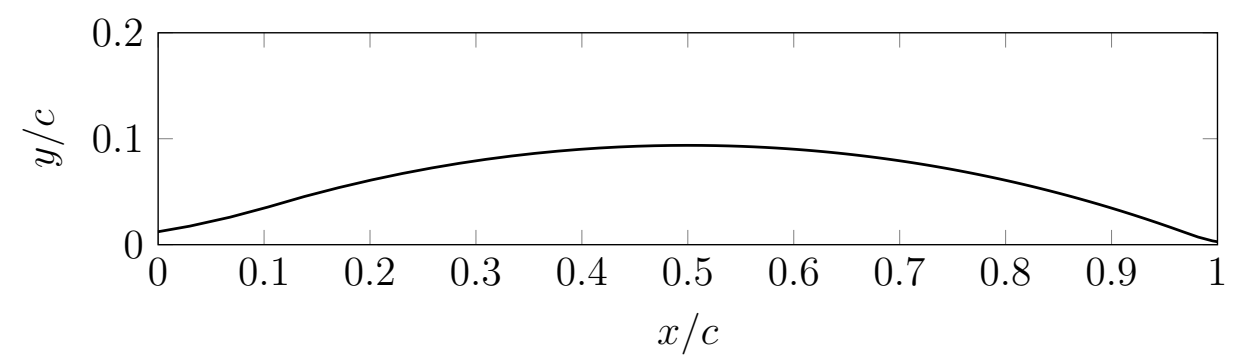

Fig. 5.2 Schematic of the transonic hump geometry normalised by the hump chord length $\mathrm{c}$

\subsection{Computational Setup}

Simulations of the axisymmetric flow are run on a sector of the cylinder and hump of angle $\theta$ using a rotated grid system about the cylinder centreline. Following this, RANS simulation are run 3D and steady using a small $\theta$. Hybrid RANS-LES are run $3 \mathrm{D}$ and unsteady using a larger $\theta$, however use a shorter extent of the upstream cylinder length to reduce computational cost. Again, a TBL is used at the domain inlet to achieve this.

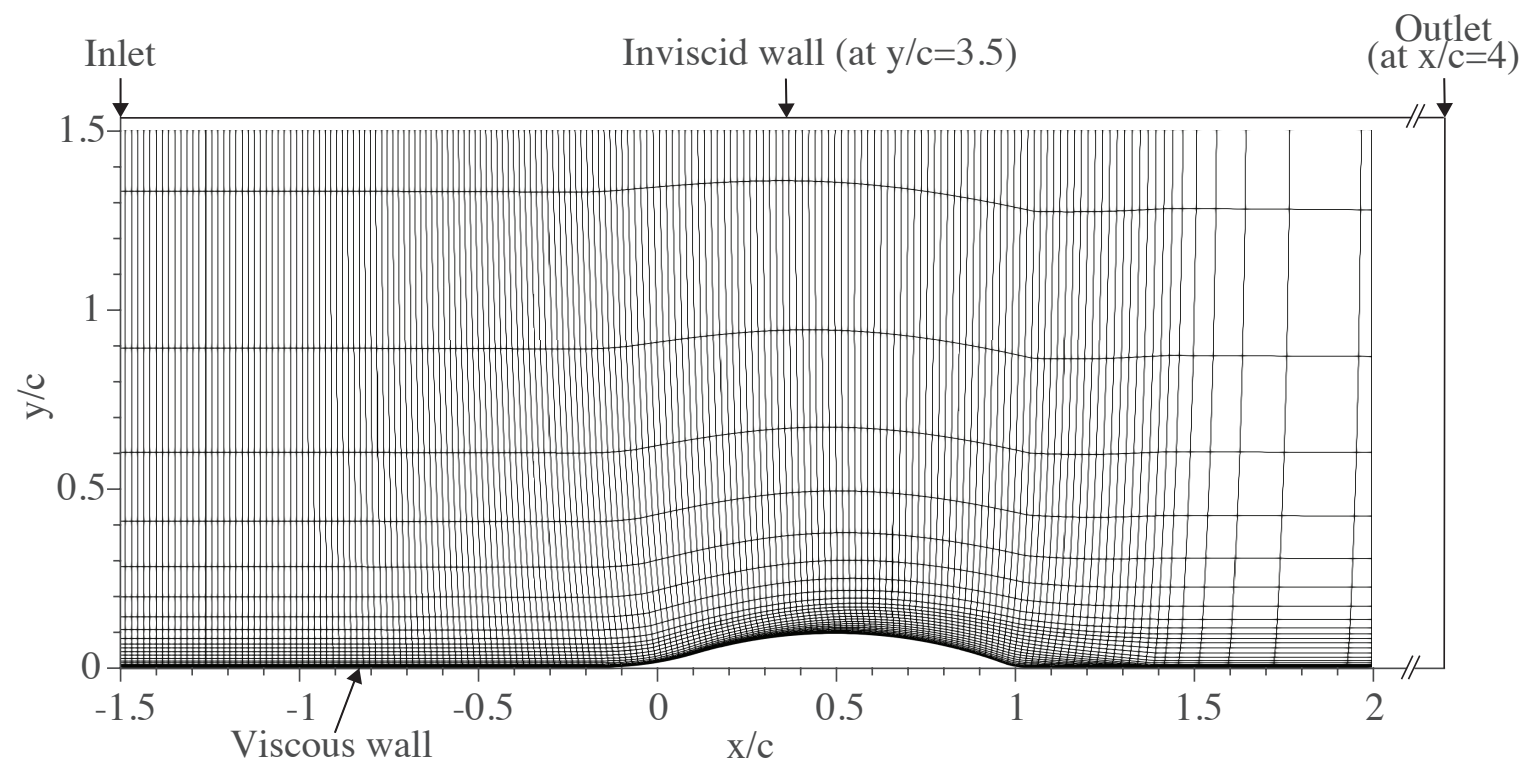

Fig. 5.3 Two-dimensional slice of the computational grid and boundary conditions used for hybrid RANS-LES of the transonic hump. Every 8th point shown in the streamwise direction and every 4 th point shown in the wall normal direction for clarity.

Figure 5.3 shows a 2D slice of the structured grid used for hybrid RANS-LES of the transonic hump. The grid extends $-1.5<x / c<4$ in the streamwise direction, 
with the hump located between $0<x / c<1$. In the wall normal direction, the grid has a height of $y / C=3.5$ from the cylinder surface at $y=0$. This corresponds approximately to the extents of the $2 \mathrm{x} 2 \mathrm{ft}$ wind tunnel walls. The grid is rotationally extruded $\theta=30^{\circ}$ about the cylinder centreline to form the $3 \mathrm{D}$ computational domain. This gives the computational domain an arc length $a \approx 0.2 C$ on the cylinder surface. Figure 5.4 shows a schematic of the computational domain used for hybrid RANS-LES simulations.

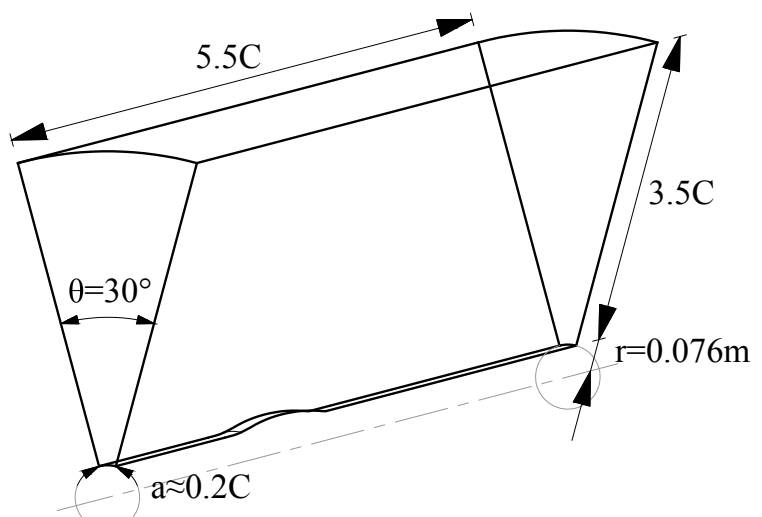

Fig. 5.4 Computational domain used for hybrid RANS-LES simulations of the transonic hump

Grid points are clustered near the shock location $x / C=0.7$ and upstream of the hump to resolve the inflow turbulent boundary layer. Downstream of the hump, the grid is gradually coarsened towards the outflow boundary. The hybrid RANS-LES grid is developed to have a grid spacing of $\Delta x^{+}=165, \Delta y^{+}=1$ and $\Delta z^{+}=100$ based on the inflow boundary layer. This is in the range of hybrid RANS-LES resolution requirements as recommended by Tucker [16] (see table 2.1). To achieve this, the grid has 1420 points in the streamwise direction, 120 points in the wall normal direction and 180 equally spaced points in the span. This gives the hybrid RANS-LES a total of 31 million points. If wall resolved LES was to be run of this case, the grid would need to be refined by at least a factor of 2 in the streamwise and spanwise directions, giving an estimated LES grid size of 125 million points.

The hybrid RANS-LES grid is defined as the baseline grid from which the RANS grid is derived. For RANS simulations, the 2D grid slice of the baseline grid is rotationally extruded $\theta=1^{\circ}$ with 3 points to form the RANS computational domain. This makes the RANS simulations relatively cheap as the time averaged axisymmetric flow is uniform around the cylinder. In addition, the upstream extent is increased from $-1.5 C$ to $-3 C$ to match the upstream length of the cylinder, allowing the RANS 
boundary layer to naturally develop.

The boundary conditions used for the cylinder surface and hump are no-slip viscous walls, the top wall is modelled as an inviscid wall, the outflow is a fixed pressure outlet and periodic boundary conditions are applied at the spanwise extents. At the inlet, a uniform inflow boundary condition is applied for RANS simulations, with the freestream velocity $M_{\infty}=0.875$. Hybrid RANS-LES simulations use an unsteady turbulent boundary layer of $R e_{\theta}=9800$ and thickness $\delta=0.0042 m(0.021 C)$ at the inflow, generated using the method described in section 3.6. As with the NASA hump, the RANS solution is used to obtain the $\operatorname{Re}_{\theta}$ and $\delta$ needed here since no experimental data is available at the inflow plane at $x / c=-1.5 C$. A velocity profile is measured by experiments at $x / c=-0.25$, and as is shown in figure 5.5, both RANS and hybrid RANS-LES agree well with measurements here.

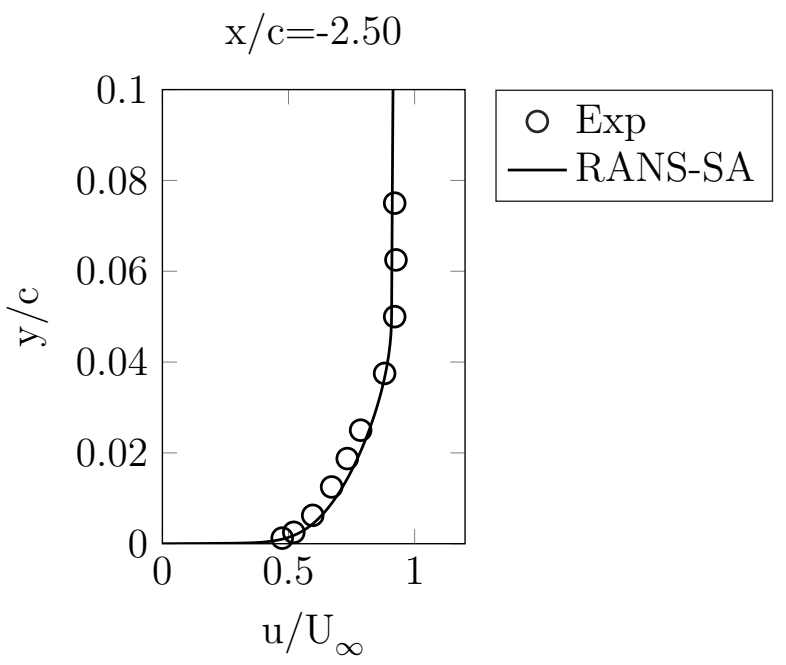

Fig. 5.5 Boundary layer velocity profile at $x / c=-0.25 C$ obtained from RANS-SA compared to experimental measurements.

RANS simulations are run using the HYDRA steady flow solver with four levels of multi-grid. Hybrid RANS-LES simulation are run using the explicit unsteady solver, using converged RANS solutions to initialise the flow. The $\sigma-S G S$ model is used here in the LES part of the domain, coupled with the modified RANS-SA model near the wall. The flow is advanced $3 T^{*}$ before flow statistics are collected. After this, time averaged flow statistics are collected over approximately $6-8 T^{*}$. For analysis, the solutions are further spatially averaged in the spanwise direction. 


\section{$5.3 \quad$ Results}

Figure 5.6 shows Mach number contours of flow over the transonic hump using the RANS-SA model. Subsonic flow initially approaching the hump encounters an adverse pressure gradient at the hump leading edge, before accelerating to supersonic above the hump body. This terminates with a normal shock wave towards the trailing edge of the hump, experimentally measured at $x / c=0.7$. The adverse pressure gradient imposed by the shock, as well as deceleration of flow here, causes flow separation. Reattachment on the cylinder surface is estimated by experiments to occur near $x / c=1.1$.

As shown in figure 5.6, RANS-SA predicts a well defined supersonic region over the hump and a well resolved normal shock. RANS-SA predicts a shock strength (maximum Mach number across the shock) of $\mathrm{M}=1.25$, which agrees well with experimental measurements estimated from the surface pressure of $\mathrm{M}=1.3$.

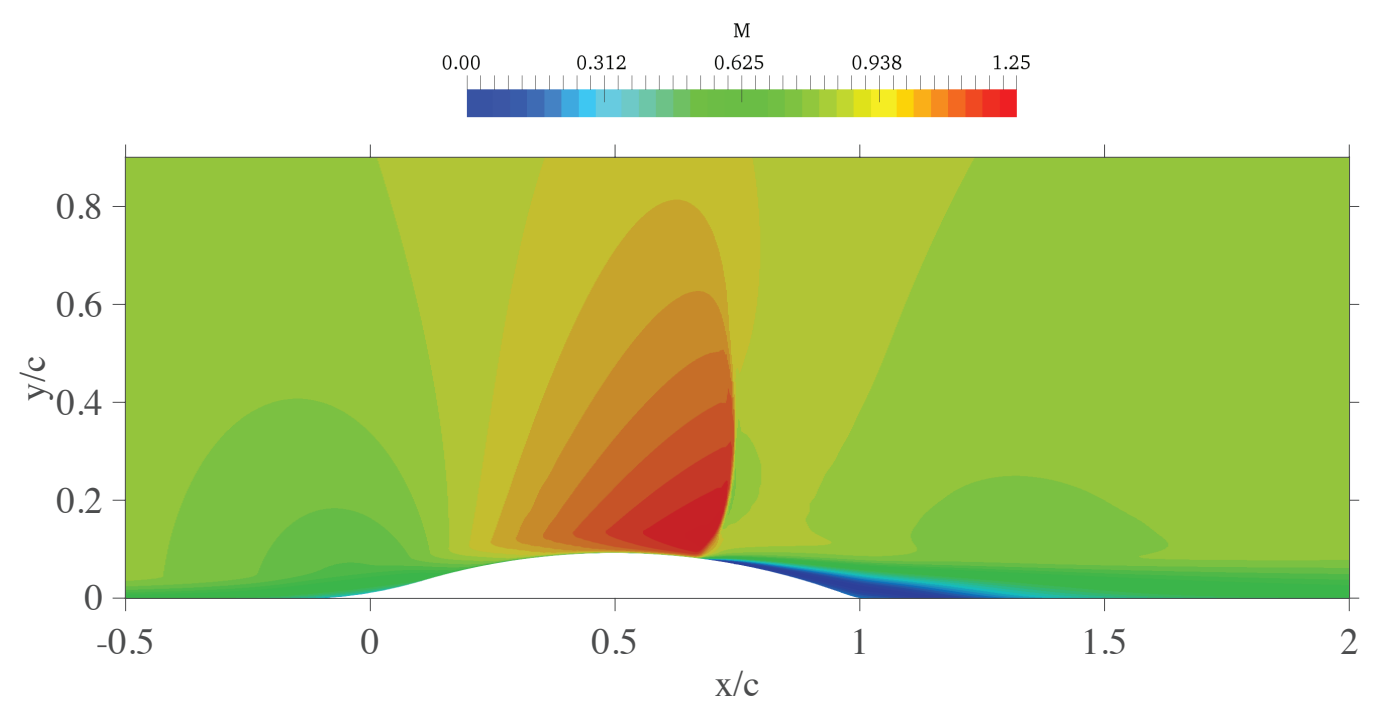

Fig. 5.6 Mach number contours of flow over the transonic hump

Figure 5.7 shows instantaneous Q-criterion contours from hybrid RANS-LES over the transonic hump. The figure shows the oncoming inflow turbulent boundary layer as it is convected over the hump body, as well as turbulent eddies in the separation. The boundary of the supersonic region is shown with a grey iso-surface at $M=1$. The turbulent eddies can be seen to stretch axially as they are accelerated over the hump, and underneath the supersonic flow. These are then shown to abruptly decelerate and thicken at the foot of the shock as they pass through the SWBLI and the flow separates.

Figure 5.8 compares the classical Jameson shock sensor and the improved Ducros 


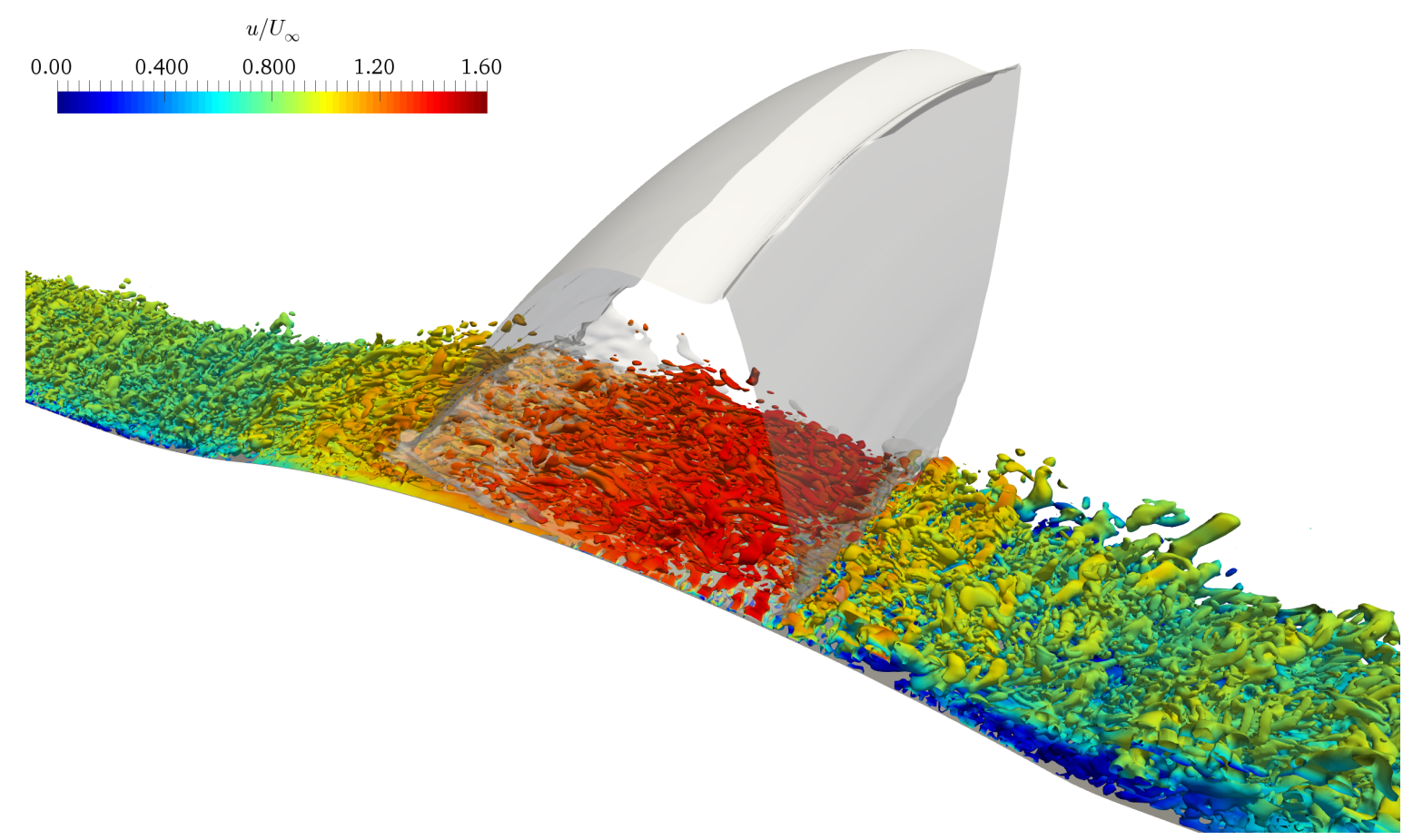

Fig. 5.7 Instantaneous Q-criterion contours coloured by velocity over that transonic hump using hybrid RANS-LES with the $\sigma$-SGS model. The flow direction is left to right. Note the grey iso-surface showing the boundary of the supersonic region $(M=1)$

sensor on the solver smoothing field in the vicinity of the hump trailing edge used for hybrid RANS-LES. Note that the Jameson sensor field is only computed for visualisation purposes, and the Ducros sensor used for actual simulations. As shown, the Jameson sensor has a high value in regions of turbulent fluctuations as well as the shock. This would lead to turbulent fluctuations being destructively smoothed by the solver. The Ducros sensor is able to provide a significant improvement here, isolating the shock and preserving the solution accuracy.

Time-averaged $C_{P}$ distributions over the transonic hump using RANS and hybrid RANS-LES are compared to experimental measurements in figure 5.9. Measurements show an abrupt rise in $C_{P}$ at $x / c=0.7$ where the shock is located, followed by a gradual recovery in the separated region and downstream of reattachment. A small deflection in $C_{P}$ is present at $x / c \approx 1.1$ which is near to the reattachment point.

The RANS-SA model predicts a $C_{P}$ distribution in very good agreement to measurements. This includes the shock location and $C_{P}$ deflection at the reattachment location. The only discrepancy found is a marginal over-prediction of the pressure in the separated region $(0.7<x / c<1.1)$. This is opposite to what was found for the NASA hump, where the pressure in the separation was under-predicted indicat- 
(a) Jameson

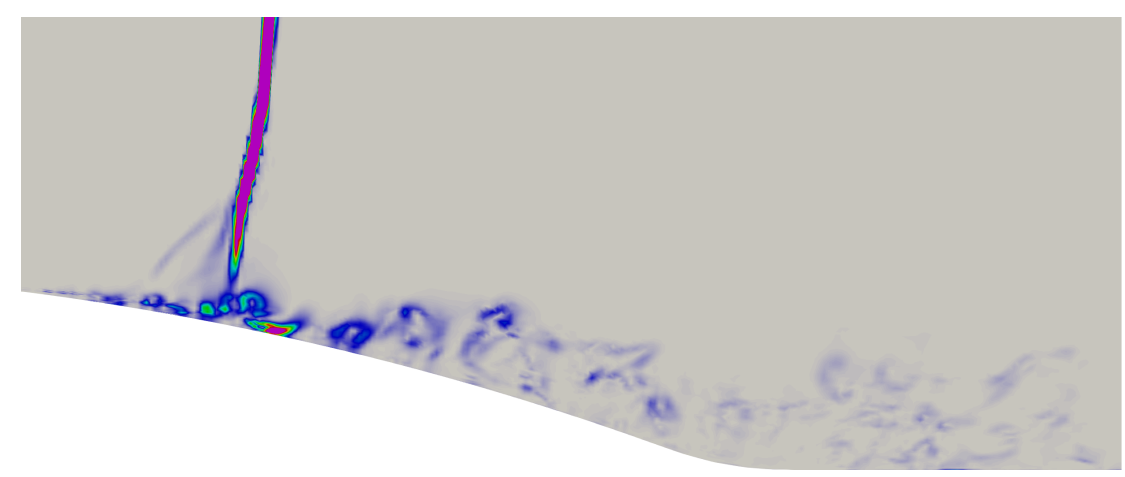

(b) Ducros

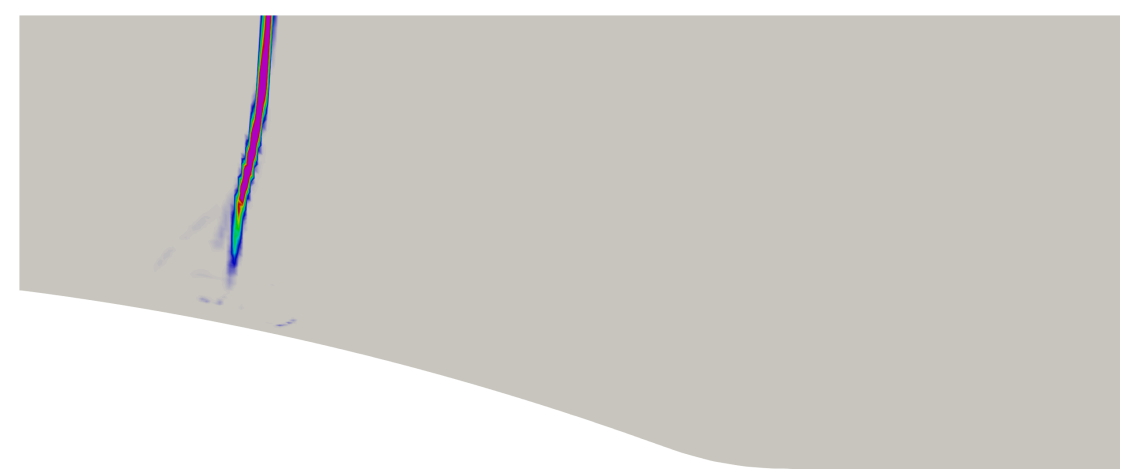

Fig. 5.8 Comparisons of the classical Jameson shock sensor and the improved Ducros sensor

ing a larger bubble. Table 5.1 shows the separation, reattachment and bubble size predicted for this flow. Note that experimental values for these were estimated from surface pressure and velocity profiles, and so these should be considered approximations. RANS-SA predicts a bubble size $17.5 \%$ larger than measurements. The good performance of RANS here can be attributed to its correct prediction of the shock location (and therefore the shock induced separation point) and the flow geometry. At the point of separation $(\mathrm{x} / \mathrm{c}=0.7)$, the slope of the hump surface means that the trajectory of the velocity vector (or flow angle) at the outer edge of the viscous layer is already directed towards the cylinder surface. This results in the separated shear layer angled towards the wall which is found to largely determine the reattachment point. Thus, since reattachment in this case is not primarily driven by turbulence, RANS is able to perform well here. Modifications to the RANS-SA for relaminarisation and curvature have little effect for this flow, with RANS-SA Mod performing very close to standard RANS-SA. 
From figure 5.9, hybrid RANS-LES generally predicts the $C_{P}$ distribution well, although there is some disagreement with the profile shape compared to experimental measurements. Hybrid RANS-LES shows a shock location that is slightly downstream compared to RANS and also fails to capture the pressure deflection at $x / c \approx 1$.1. From table 5.1, we see hybrid RANS-LES separates $0.02 \mathrm{c}$ later and reattaches $0.03 \mathrm{c}$ earlier than measurements, predicting a bubble size $12.5 \%$ shorter than measurements.

The reasons for hybrid RANS-LES disagreements here can be attributed to its delayed shock location. Although small (only 3\% chord further downstream than RANS), the fact that separation is shock induced means flow separation occurs later on the hump body where the flow gradient towards the cylinder surface is greater. This results in the flow being forced to reattach sooner, therefore giving a smaller bubble and faster pressure recovery compared to measurements. While this can be considered specific to this geometry, it does demonstrates the importance of predicting shock location accurately, where even small deviations can affect the flow downstream.

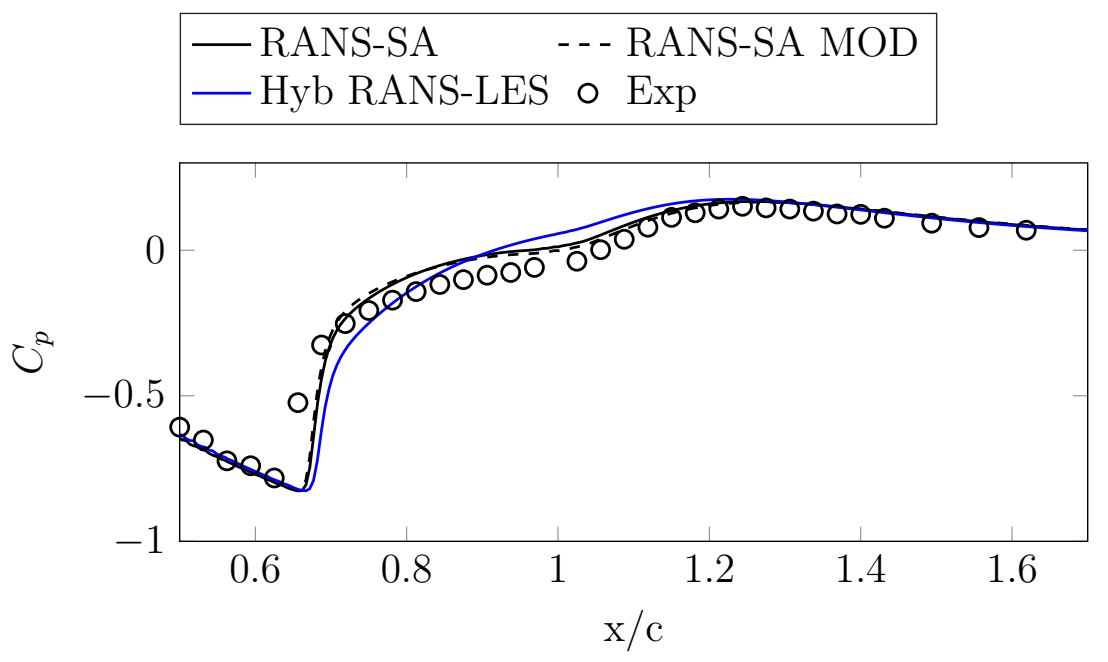

Fig. $5.9 C_{p}$ distribution over the transonic hump

\begin{tabular}{|l|l|l|l|}
\hline & $\begin{array}{l}\text { Separation } \\
\text { Point }(\mathrm{x} / \mathrm{c})\end{array}$ & $\begin{array}{l}\text { Reattachment } \\
\text { point }(\mathrm{x} / \mathrm{c})\end{array}$ & $\begin{array}{l}\text { Bubble } \\
\text { size }(\mathrm{x} / \mathrm{c})\end{array}$ \\
\hline Exp & $\approx 0.7$ & $\approx 1.1$ & $\approx 0.4$ \\
\hline RANS-SA & 0.69 & 1.16 & 0.47 \\
\hline RANS-SA Mod & 0.69 & 1.16 & 0.47 \\
\hline Hyb RANS-LES & 0.72 & 1.07 & 0.35 \\
\hline
\end{tabular}

Table 5.1 Separation, reattachment and bubble size over the transonic hump 
Figure 5.10 shows axial velocity profiles at 6 locations downstream of the shock compared to experimental measurements. As with surface pressure, RANS is able to give a good prediction at all locations, with the profile shapes matching well with measurements. We can however see RANS showing flow reversal at $x / c=1.125$ where measurements do not, displaying its slight over prediction of the separation. Hybrid RANS-LES is also able to predict good profile shape, although its smaller separation size is evident here. The height of the velocity deficit is closer to the wall at all locations since the bubble height is predicted smaller. We can also see the sharp shear flow shown by hybrid RANS-LES at the first profile location, indicating the delayed separation as the separated shear layer is still well defined here. Nevertheless, profiles at the last two locations, where the flow is now attached, show good agreement.
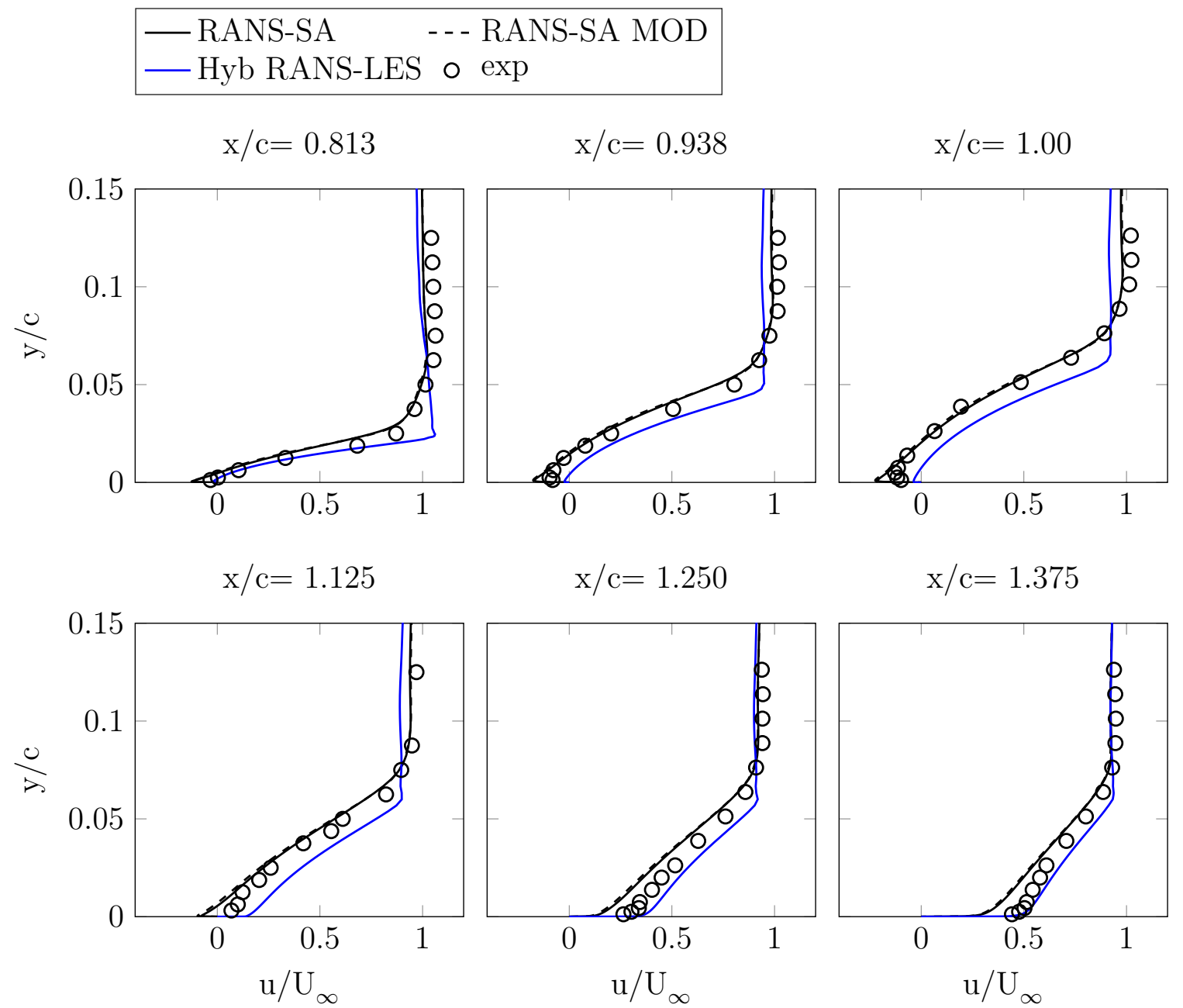

Fig. 5.10 Streamwise velocity profiles located downstream of the shock separation compared with experimental measurements for RANS and hybrid RANS-LES 
Figure 5.11 shows resolved TKE and $\overline{u^{\prime} v^{\prime}} / U_{\infty}^{2}$ profiles obtained from hybrid RANSLES at the same 6 locations downstream of the shock compared to experimental measurements. For both TKE and $\overline{u^{\prime} v^{\prime}} / U_{\infty}^{2}$, hybrid RANS-LES is able to predict peak turbulence levels with very good agreement to measurements except at the first location. Here, turbulence is significantly over-predicted since this is closer to hybrid RANS-LES's delayed separation point. Profile shapes at all other locations agree well, although like velocity, the peaks are skewed towards the wall for $1.00<x / c<1.125$ since the bubble is predicted smaller. At the last two locations however, where the flow is now attached, a very good prediction of the downstream turbulence is obtained. This is encouraging for hybrid RANS-LES simulations.

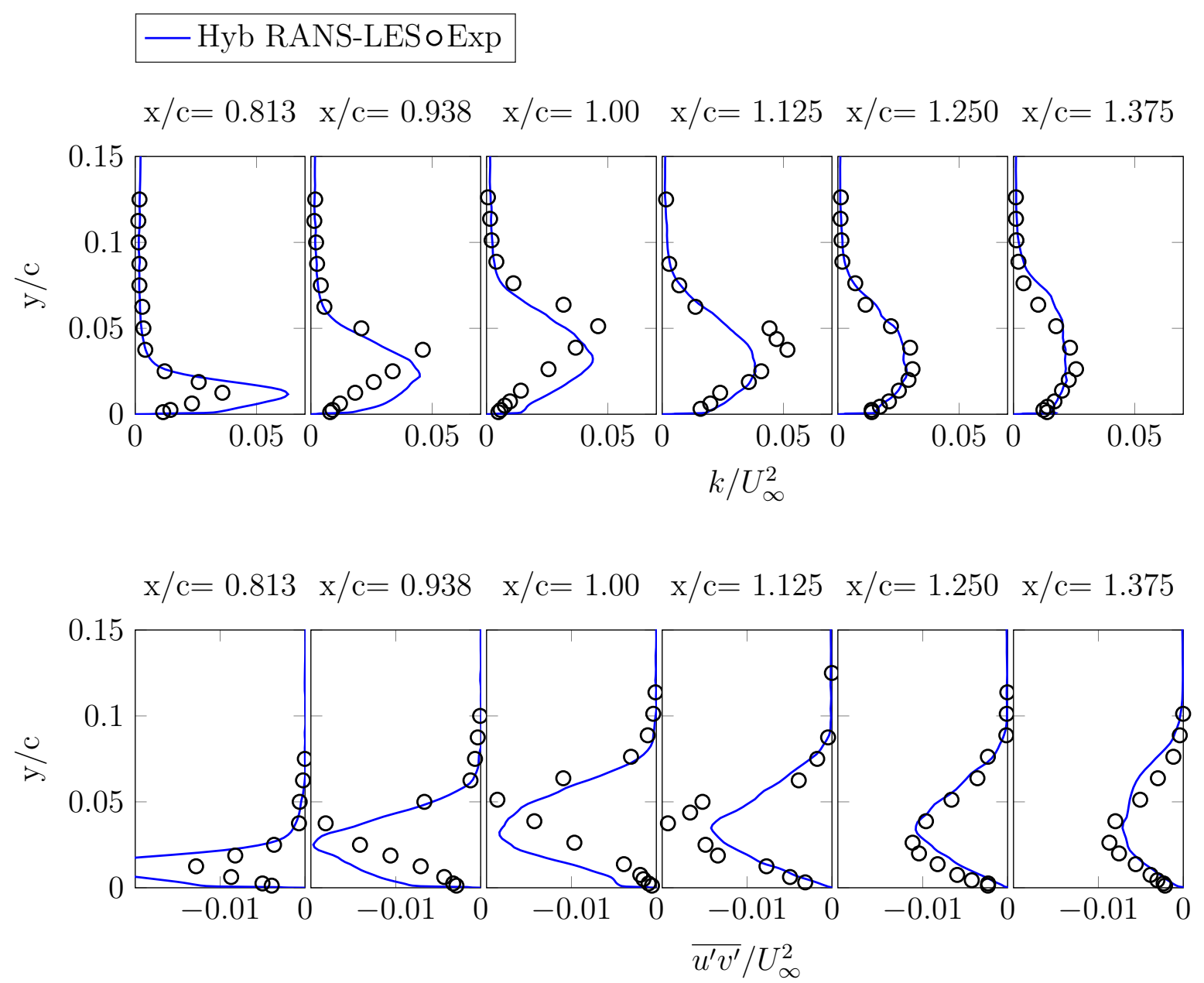

Fig. 5.11 Turbulent kinetic energy and Reynolds stress profiles located downstream of the shock separation compared with experimental measurements for hybrid RANSLES 
Hybrid RANS-LES can be used to study additional turbulence behaviour through the SWBLI. Experiments report the upstream influence of the shock imposes a deceleration of the flow in the low momentum inner boundary layer leading up to the interaction. This is then subjected to a large perturbation at the shock that causes a rapid increase in the Reynolds shear stress here. Figure 5.12 shows Reynolds stress profiles from hybrid RANS-LES at a fixed height of $0.005 \mathrm{c}$ above the hump surface, corresponding to $\approx 1 \mathrm{~mm}$. Upstream of the SWBLI, the relatively low level of turbulence content can be seen in this region. At the point of the interaction $(x / c \approx 0.7)$, the figure shows this sudden amplification of turbulence as the near wall flow passes through the SWBLI. $\overline{u^{\prime} u^{\prime}} / U_{\infty}^{2}$ in particular is shown to undergo a dramatic amplification, almost 3 times $\overline{v^{\prime} v^{\prime}} / U_{\infty}^{2}$. This displays the instabilities shocks can impose on the near wall viscous flow. Downstream of this, turbulence levels gradually decrease towards the separation.

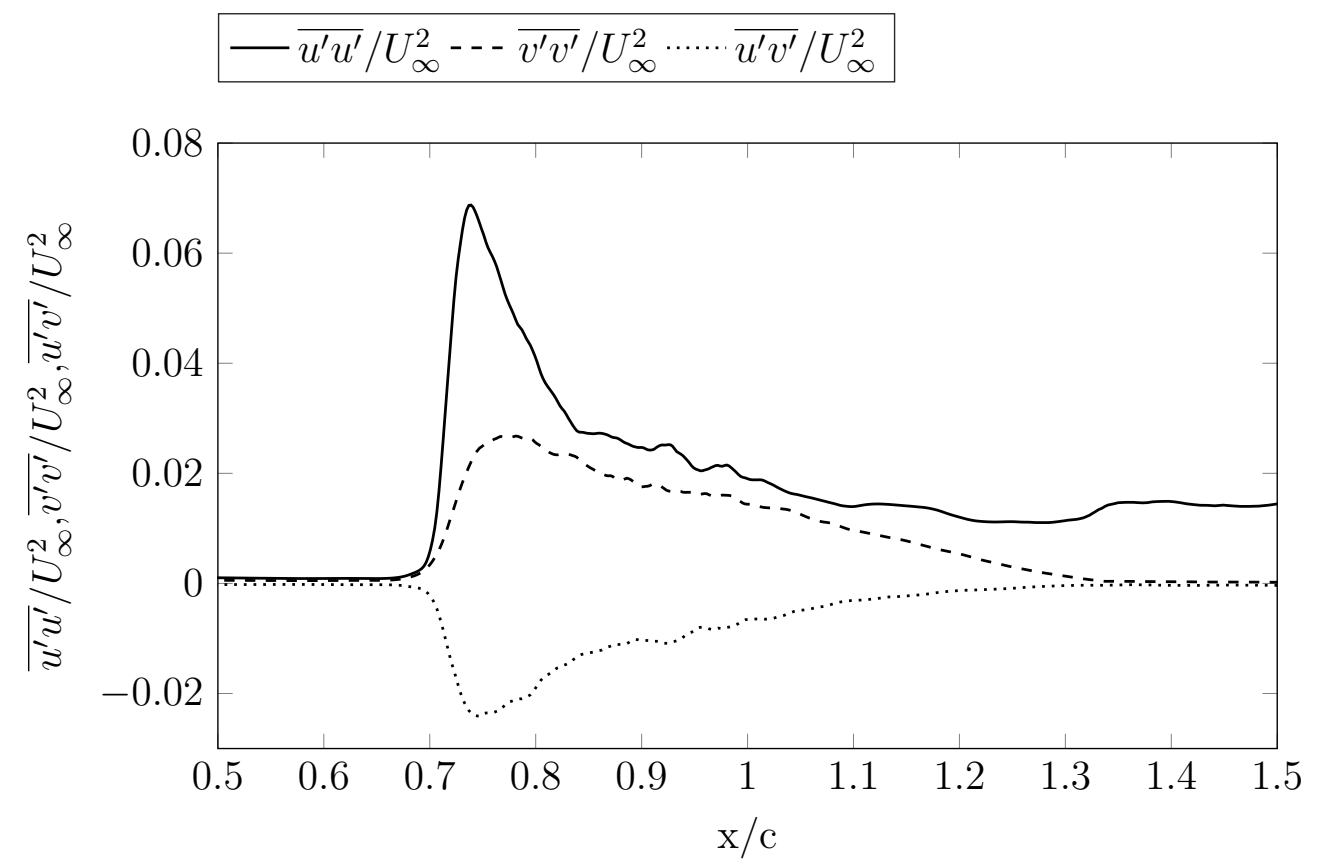

Fig. 5.12 Reynolds stress profiles at a fixed height of 0.01c above the hump surface

\subsection{Conclusions}

RANS predictions of transonic flow over an axisymmetric hump model show very good agreement with experimental data. The SWBLI is turbulent in nature and is strong enough to cause flow separation. RANS is able to predict the shock location well. The 
trajectory of flow towards the wall allows RANS to also predict the separated flow region well for this case. This overall agrees with literature, whereby RANS methods work well at predicting shock location and mean surface pressure for steady SWBLIs without large separations.

Hybrid RANS-LES simulations of this case are also able to predict mean flow quantities well. The use of the Ducros sensor substantially improves the solver's numerical smoothing field, isolating the shock where the standard Jameson sensor fails. However, hybrid RANS-LES predicts a shock location downstream compared to measurements. While this is a relatively small delay, the geometry of the hump model means that flow separation size is under-predicted. This demonstrates the importance of predicting shock location accurately, where even small deviations can affect the flow downstream. This must therefore be an important consideration for SWBLIs in engine intake.

Nevertheless, hybrid RANS-LES does enable additional study of turbulence through the SWBLI and separation. This is able to predict Reynolds stress magnitudes well compared to measurements. Simulations are also able to show the rapid amplification of turbulent stresses experienced by the inner boundary at the point of the SWBLI. Overall, this is encouraging for hybrid methods where fully wall resolved LES simulations are prohibitively expensive. 



\section{Chapter 6}

\section{Intake Rig Model}

\subsection{Introduction}

The final chapter of this project will use the CFD methods explored in previous chapters to simulate an experimental intake rig model. The rig is designed with the aim of finding a simple geometric configuration that replicates the key physics found in a real intake at incidence, allowing study of such phenomena and providing experimental validation data sought for by computational models. The intake rig was designed and built by fellow PhD student Tafara Makuni [15, 21] and experimental measurements used in this work were made by fellow PhD student Andrea Coschignano [23, 24], both under the supervision of Professor Holger Babinsky at the Department of Engineering, University of Cambridge. The motivation for this study and the experimental effort is driven by a lack of previous research both experimentally and computationally that investigates transonic high incidence lip flows found in intakes at incidence (as found in the literature review). The novel intake rig model is believed to be the only known setup of its kind.

The rig model was developed in parallel to this work with the partnering of projects providing many advantages. Preliminary CFD simulations were able to guide the design of experimental geometry, such as the shape of the wind tunnel liners. The close proximity of computational and experimental work also enabled new measurements to be made in real time, such as stagnation point location needed for CFD validation. This overall enabled confidence in both experimental and computation work.

RANS, LES and hybrid RANS-LES are used for simulations of the intake rig. The acceleration and flow curvature over the intake lip are high akin to a real intake and so the relaminarisation and SARC modifications to the SA model are expected to be very active here. The cases tested also possess varying degrees of shock oscillation which the 
unsteady simulations (LES and hybrid RANS-LES) aim to capture. The nature of the SWBLI is also transitional, where shock induced transition occurs on the lip surface. Reproduction of the flow downstream of the lip (after the shock and SWBLI) is of particular interest as it is this flow that would be propagating towards the engine fan in a real intake. The ability of the simulation methods to accurately predict this will be assessed. The chapter aims to conclude this work by recommending a simulation strategy for intakes at incidence based on computational cost and performance of simulation methods.

\subsection{Case details}

The intake rig experimentation is carried out in the small-scale blow-down supersonic wind tunnel II (SST2) of the Cambridge University Engineering Department. A brief description of the experimental design is given here. Full details can be found in Makuni [15].

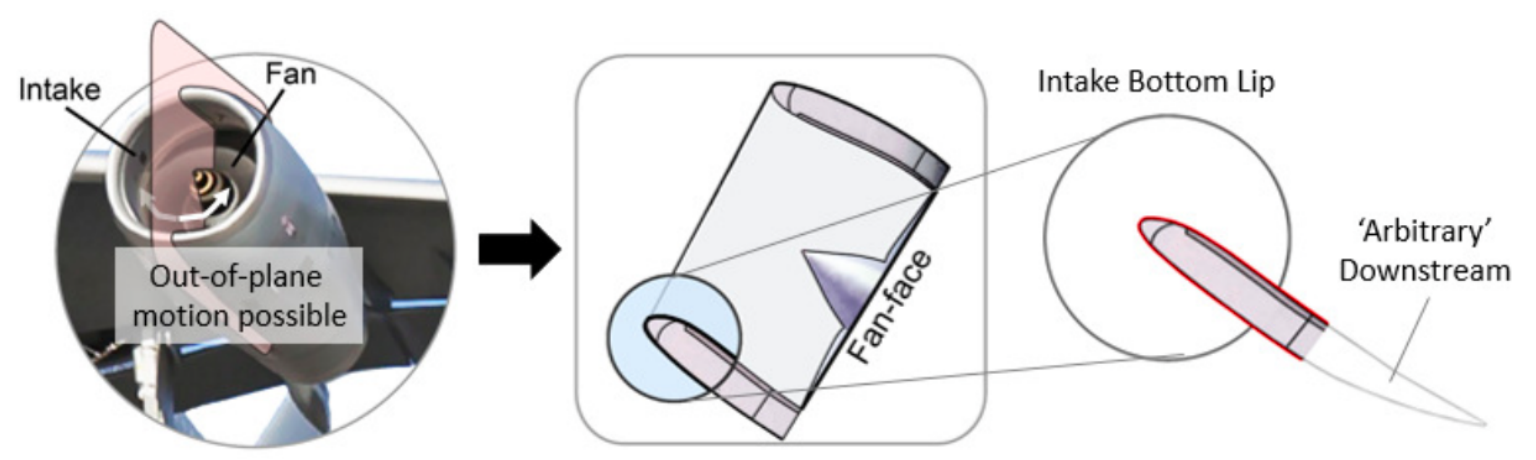

Fig. 6.1 Simplification of a real intake to a two dimensional aerofoil. Figure from Makuni [15]

The experiment is designed around a two dimensional representation of an intake lip based on the Rolls-Royce Trent 700 intake. The two dimensional approach makes it easier to obtain high quality measurements, as well as simplifying the problem for computations. The profile is chosen to match the bottom dead centre of the real three dimensional intake lip, where the SWBLI is believed to be strongest. An arbitrary downstream section is added to the intake profile that is chosen purely to impose minimum disturbance on the flow upstream in the region of interest (i.e. the lip). As such, the geometry resembles that of an aerofoil, and will be referred as such herein. Fig. 6.1 illustrates the 3D intake to 2D aerofoil simplification process.

Since the original straight working section of the SST2 wind tunnel (designed for 


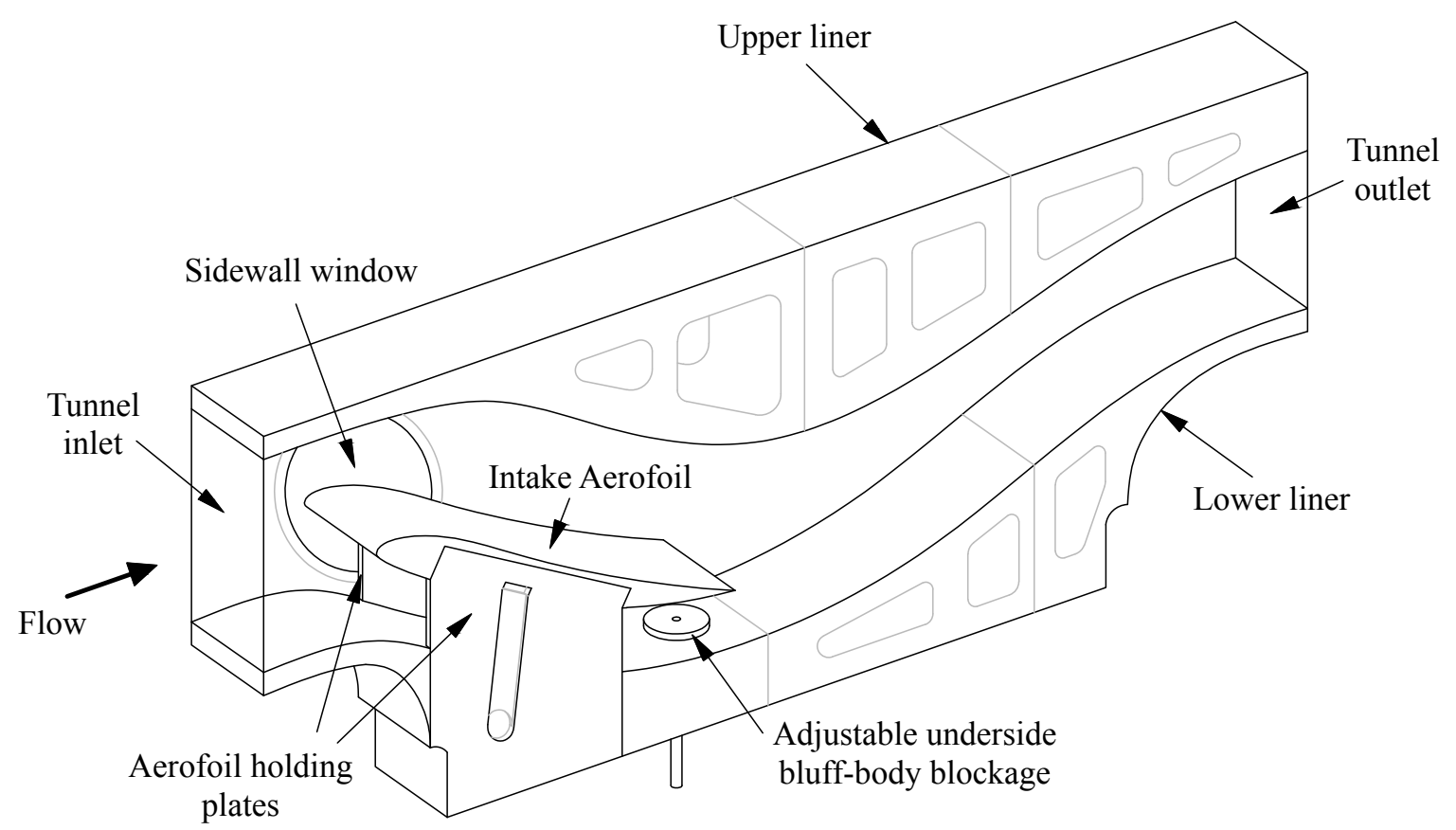

Fig. 6.2 Schematic of the intake rig working section and key components

zero incidence flows) is unsuitable for a high incidence problem, a new flow path and working section resembling a shallow v-shape are designed and built. Figure 6.2 shows a schematic of the rig working section, with the key parts labelled. The intake aerofoil spans the entire width of the tunnel. This results in unavoidable sidewall effects not present in a real intake, such as 3D corner separation where the aerofoil meets the tunnel sidewall. However, these are measured to be small and confined to near the wall, with the central tunnel flow largely two dimensional (this is shown later in figure 6.4). It should also be noted that the aim of the work is to reproduce the main features of the flow fields found in a real intake at high incidence, and not the exact flow field of a particular intake.

The entry Mach number and overall mass flow that define the global parameters of the rig are controlled via a variable area diffuser downstream of the working section. In addition to this, an adjustable height bluff body blockage mechanism is located on the lower wind tunnel liner, underside the aerofoil at approximately $80 \%$ chord. This is also shown in figure 6.2. This mechanism is installed in order to control the local flow over the lip. By increasing the underside blockage, the mass flow over the upper side of the aerofoil (and consequently the local Mach number here) can be increased, allowing varying shock intensities to be tested while maintaining the global flow parameters. Note that this in essence enables control of the stagnation 
point location on the lip as it is the dividing point between the flow that travels above and below the aerofoil. Increasing the underside blockage moves the stagnation point aft towards the aerofoil trailing edge, while decreasing it moves the stagnation point forward towards the leading edge.

The intake aerofoil has chord length of $C=0.445 \mathrm{~m}$, maximum lip thickness $L=$ $0.055 \mathrm{~m}$ and span $s=0.114 \mathrm{~m}$. $L$ is used as the characteristic dimension of the flow. The inlet of the tunnel working section is $2.71 \mathrm{~L}$ upstream of the aerofoil leading edge and has a height of $4.16 \mathrm{~L}$. Two angles of attack of the intake aerofoil are investigated, $\alpha=23^{\circ}$ and $\alpha=25^{\circ}$. These produce significantly different flow characteristics. The baseline flow $\alpha=23^{\circ}$ produces a stable steady shock, with the SWBLI strong enough to induce a small separation bubble followed by flow reattachment and recovery towards the trailing edge. The more aggressive $\alpha=25^{\circ}$ on the other hand produces an unstable shock with shock oscillation. The resulting SWBLI gives a larger separation and heavily distorted flow towards the trailing edge.

The freestream conditions are measured by a probe located approximately $2 L$ upstream of the aerofoil. For both angles of attack, the freestream Mach number is set to $M_{\infty}=0.435$, giving a Reynolds number based on the lip thickness of $R e_{L}=$ $5.5 \times 10^{5}$. Freestream turbulence levels are also measured giving a turbulence intensity $T i=0.435 \%$ and turbulence length scale $l_{\text {turb }}=2 \mathrm{~mm}$, which can be considered low.

Figure 6.3 shows photos of the intake rig working section and a close up of the intake aerofoil model.

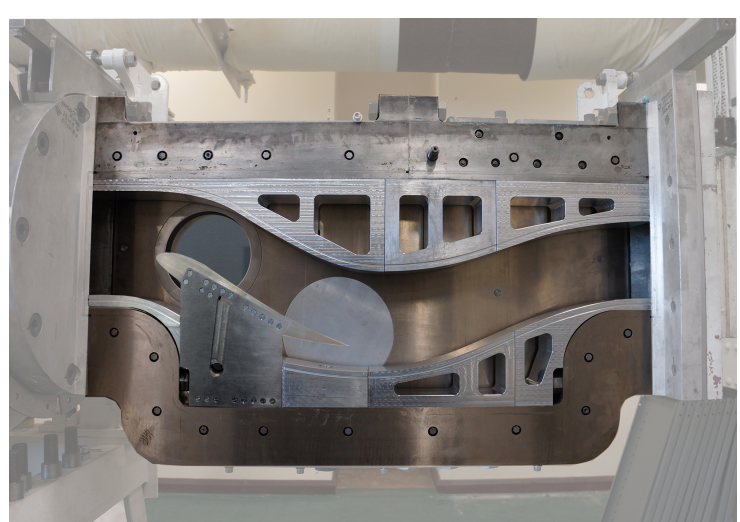

(a) Intake rig working section

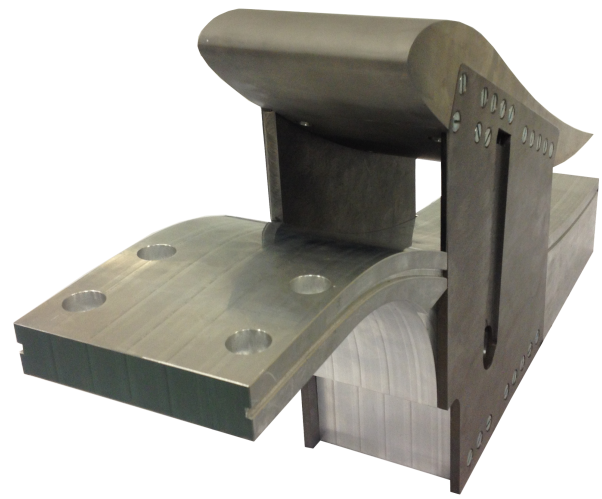

(b) Close up of the intake aerofoil model

Fig. 6.3 Photos of the Intake rig and aerofoil

For experimental measurements, high speed Schlieren photography is used to qualitatively capture the flow field over the lip (based on density gradients) using a pair 
of windows on each side of the working section. The windows also allow pressure sensitive paint (PSP) to be used to measure the pressure on the aerofoil surface. In addition, velocity profiles are measured normal to the aerofoil surface at three locations downstream of the shock for the $\alpha=23^{\circ}$ case and at one location for $\alpha=25^{\circ}$.

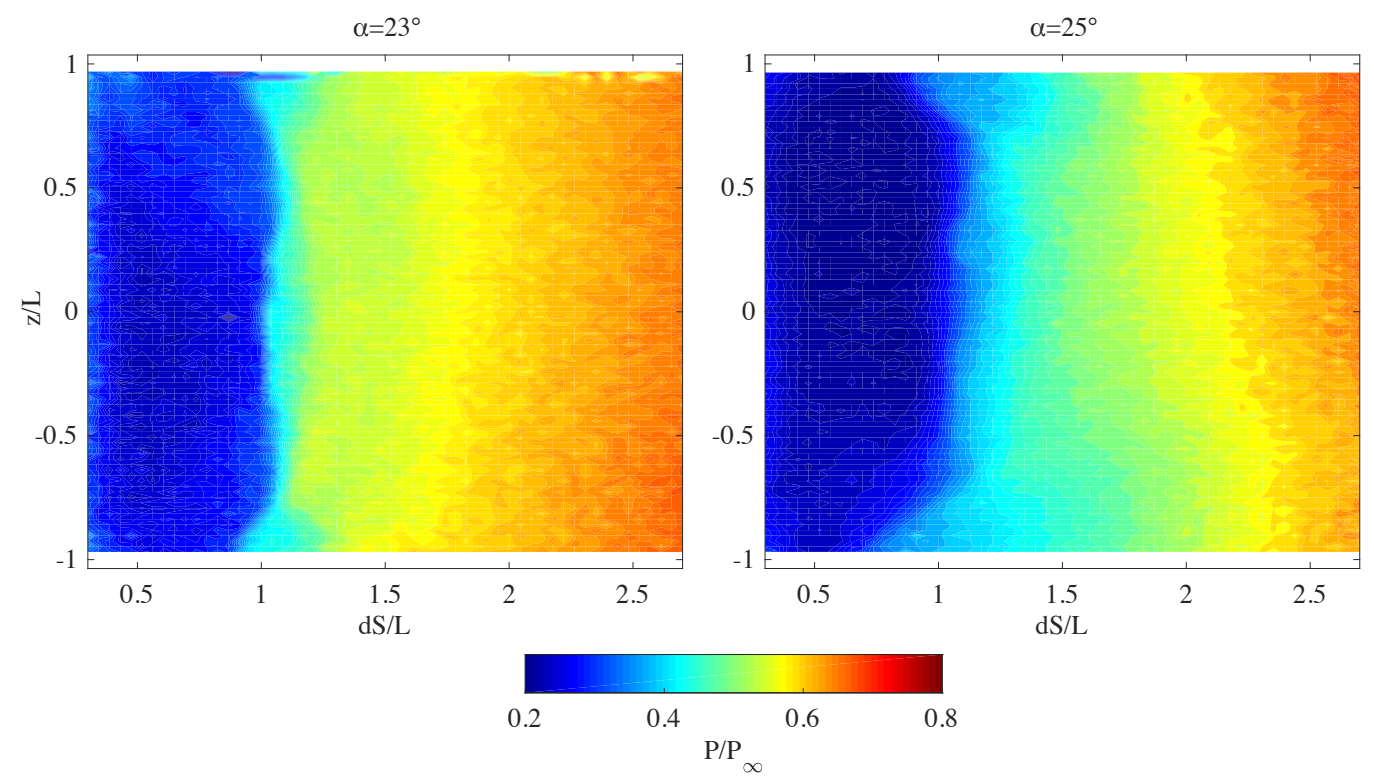

Fig. 6.4 Processed time-averaged surface pressure data captured experimentally for both $\alpha=23^{\circ}$ and $\alpha=25^{\circ}$ cases using PSP. Flow direction is left to right.

Figure 6.4 shows the processed time-averaged surface pressure data captured experimentally for both $\alpha=23^{\circ}$ and $\alpha=25^{\circ}$ cases using PSP. The plots give an indication of sidewall effects, whereby the largely two-dimensional centre span flow is smeared close to the spanwise (z) extents. For comparisons to CFD, a spacial average of the central $25 \%$ is used.

\subsection{Computational Setup}

This work considers the two dimensional centre span flow of the intake rig without sidewalls. This allows RANS computations to be efficiently run using a $2 \mathrm{D}$ steady approach. LES and hybrid RANS-LES simulations are run 3D and unsteady using approximately $25 \%$ mid span of the rig. These also use the $\sigma$-SGS model and Ducros shock sensor in the LES regions of the domain, with the modified RANS-SA model to account for laminarisation and curvature used in the inner boundary layer for hybrid RANS-LES. Since the baseline $\alpha=23^{\circ}$ case is measured to be relatively steady with small separation, only hybrid RANS-LES is run here due to limited computational 
resources. Wall resolved LES and hybrid RANS-LES are run for the more aggressive $\alpha=25^{\circ}$ case.

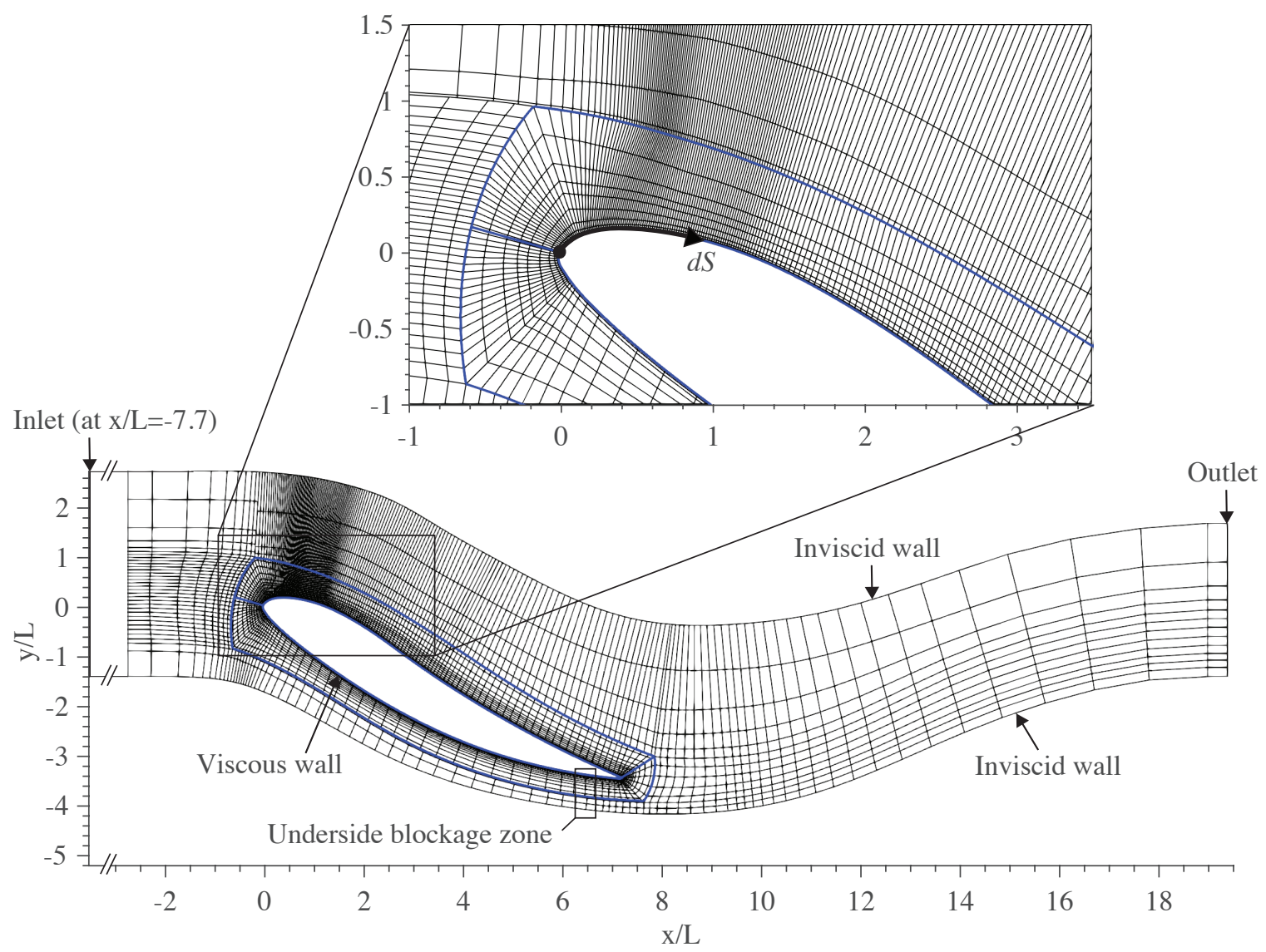

Fig. 6.5 Two-dimensional slice of the computational grid and boundary conditions used for LES of the intake rig at $\alpha=25^{\circ}$. Every 8th point shown for clarity. Inset: close up of the grid around the lip. Note the blue lines showing the O-grid around the aerofoil.

Figure 6.5 shows a two dimensional slice of the computational grid used for LES of the intake rig at $\alpha=25^{\circ}$. This is defined as the baseline grid from which RANS and hybrid RANS-LES grids are derived for both $\alpha=23^{\circ}$ and $\alpha=25^{\circ}$ cases. The grids are fully structured with a $\mathrm{H}-\mathrm{O}-\mathrm{H}$ grid topology. The sharp trailing edge of the aerofoil is modified to a rounded blunt edge to construct the O-grid by removing approximately $1 \%$ chord. This is not believed to affect the flow at the lip and reduces the excessive grid resolution downstream of the blade associated with a structured C-grid required for sharp trailing edges.

The computational domain includes the full length and height of the wind tunnel working section with the aerofoil leading edge located at $x=y=0$. Also note the 
direction $d S$ along the intake aerofoil surface as shown inset in figure 6.5. The downstream extent of the grid matches the tunnel outlet at $x / L=19.45$. The upstream extent of the grid is extended beyond the tunnel inlet by $5 L$ to ensure the numerical boundary does not influence the region of interest (the intake lip). This places the upstream extent of the grid at $x / L=-7.7$. The grid is extruded $0.5 L$ in the spanwise direction to form the 3D computational domain. This is $24 \%$ of the rig span.

The LES grid is developed to have a grid spacing of $\Delta x^{+}=60, \Delta y^{+}=1$ and $\Delta z^{+}=25$ around the intake lip and on the upper aerofoil surface up to $50 \%$ chord. This is to ensure adequate LES grid resolution in the region of interest and is in the range recommended by Tucker [16] (see table 2.1). To achieve this, the LES grid has 167,525 points per two-dimensional slice and 300 equally spaced points in the span. This gives the LES grid a total of 50 million points. Downstream of the aerofoil, the grid is gradually coarsened towards the outlet boundary to smooth flow fluctuations and prevent reflections at the boundary.

To generate the hybrid RANS-LES grids, the baseline LES grid is coarsened by a factor of approximately 2 in the streamwise and spanwise directions. This gives a grid spacing of $\Delta x^{+}=120, \Delta y^{+}=1$ and $\Delta z^{+}=50$ in the region of interest. This again is in the range recommended for hyrbid RANS-LES by Tucker [16] (see table 2.1). The hybrid RANS-LES grid has 95,000 points per two-dimensional slice and 150 equally spaced points in the span giving a total of 14 million points. For 2D RANS simulations, a two dimensional slice of the baseline grid is used as the RANS computational domain.

For all simulations, the boundary conditions used for the intake aerofoil surface is a no-slip viscous wall, the outlet is a fixed pressure outlet and the inlet is a uniform inflow boundary with $M_{\infty}=0.435$. The top and bottom wind tunnel liners are modelled as inviscid walls. This allows a reduction in computational cost as the liner boundary layers are measured to be small and sufficiently far from the region of interest. This is verified by running RANS simulations with viscous and inviscid liner walls, with both showing a negligible difference between results around the intake aerofoil.

The underside blockage (used experimentally to control the stagnation point location) is numerically modelled in simulations using a simple bodyforce model. This alleviates the need explicitly resolve the bluff body mechanism, allowing blockage levels to be changed numerically without regenerating computational domains or grids. This is implemented in the HYDRA CFD code by introducing a bodyforce term on the streamwise velocity equation to act as a numerical forcing on the flow, which is applied in a blockage zone in the underside channel at $80 \%$ chord as shown in figure 6.5 . The 
magnitude of the blockage is manually adjusted using a scaling factor to obtain the desired stagnation point location on the aerofoil to match experiment. The baseline $\alpha=23^{\circ}$ has an underside blockage to give a stagnation point of $d S=-0.0146 \mathrm{~m}$ on the intake lip. No underside blockage is used for $\alpha=25^{\circ}$ (as is also the case in experiments).

RANS simulations are run using the HYDRA steady flow solver with four levels of multi-grid. LES and hybrid RANS-LES simulation are run using the explicit unsteady solver, using converged RANS solutions to initialise the flow. The $\sigma-S G S$ model is used in the LES part of the domain, coupled with the modified RANS-SA model near the wall for hybrid RANS-LES. The flow is advanced $1 T^{*}$ before flow statistics are collected, where $T^{*}$ is the flow-through time, defined $T^{*}=C / U_{\infty}$. This allows initial transients to be flushed from the region of interest. After this, time-averaged flow statistics are collected over approximately $5-12 T^{*}$. For analysis, the solutions are further spatially averaged in the spanwise direction.

\subsection{Results}

\subsection{1 $\alpha=23^{\circ}$ Case}

Figure 6.6 shows the static pressure distribution around the aerofoil using the RANS SA model, displaying the effect of the numerical blockage applied in the intake underside passage. The volume source term effectively creates an adverse pressure gradient in the blockage zone, allowing the mass flow split through the upper and lower channels of the rig to be controlled. The method performs well at efficiently reproducing the experimental underside blockage control, and does not present any simulation stability or convergence issues.

Although not further investigated in this work, figure 6.7 shows the effects of varying the underside blockage for $\alpha=23^{\circ}$ on the local Mach number distribution over the aerofoil lip. As shown, a low underside blockage produces a weaker shock and an upstream shock location, while a high blockage moves the shock downstream and increases the shock strength and supersonic region size. The figure shows how the implemented numerical blockage method is able to reproduce this well comparing to experimental schlieren images. We can also note under high blockage in fig. $6.7 \mathrm{c}$ a strong SWBLI where shock induced flow separation completely detaches the flow. This appears to be consistent with the experiment.

Simulations are able to reproduce the key flow features of the intake rig flow. Figure 


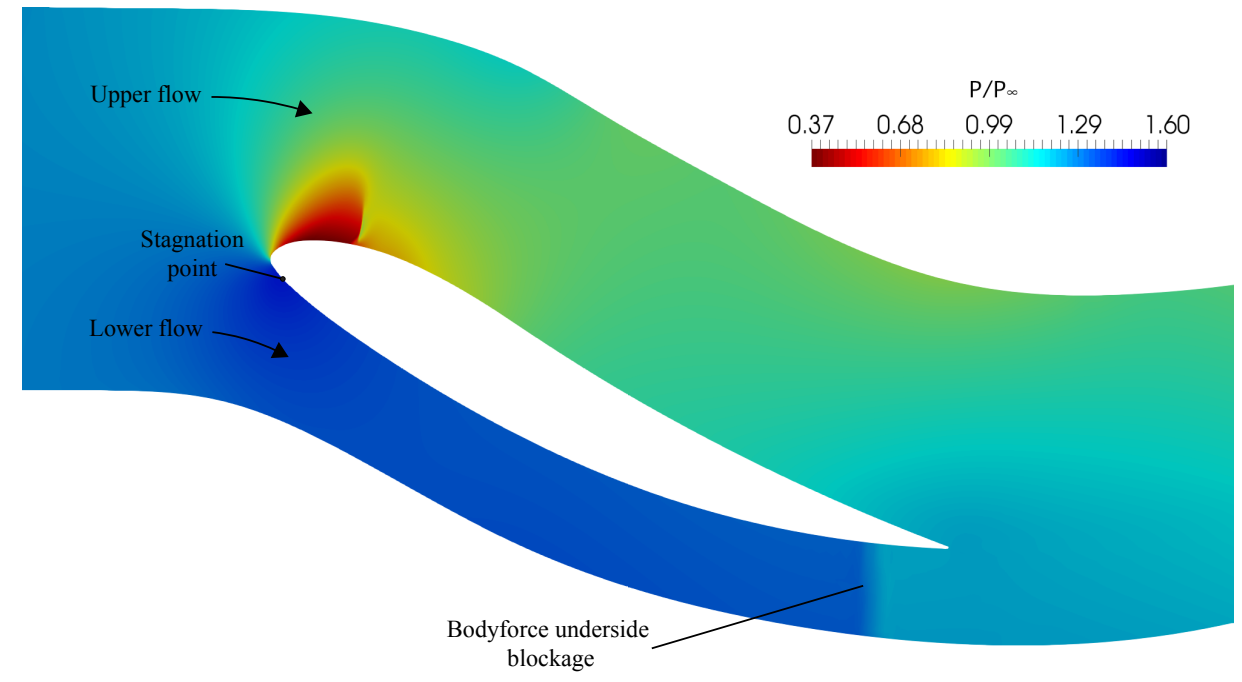

Fig. 6.6 Static pressure distribution around the aerofoil using the RANS SA model
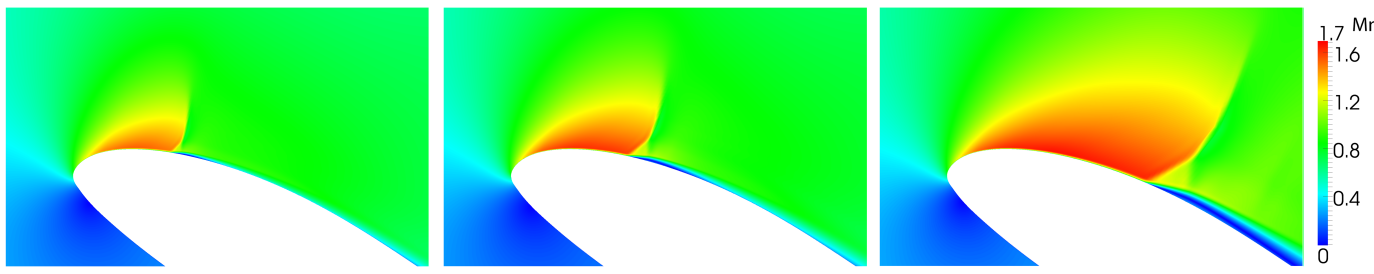

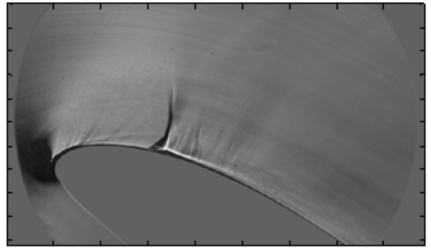

(a) Low blockage

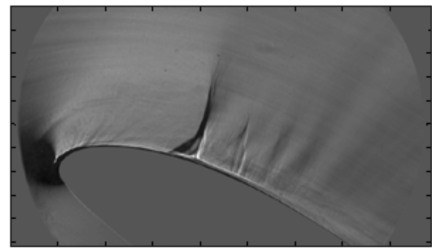

(b) Design case

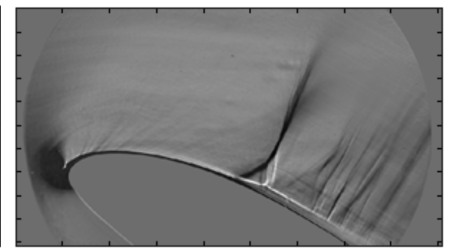

(c) High blockage

Fig. 6.7 Effects of varying underside blockage on the lip flow. Top: CFD using the RANS SA model, bottom: experimental schlieren images

6.8 shows a close-up of the baseline intake flow around the lip from experimental Schlieren imaging and CFD (Schlieren-like contours of $d \rho / d y$ ) using the standard RANS-SA model. The shock strength (maximum Mach number across the shock) for $\alpha=23^{\circ}$ is $M=1.3$. Simulations produce a well defined supersonic region terminating with a well resolved near normal shock wave. We can also see the presence of the $\lambda$ shock foot and SWBLI that display the presence of a small separation bubble just downstream of the shock wave. These qualitatively agree well with experiment, with flow features within agreeable sizes and locations. This is expected of the baseline $\alpha=23^{\circ}$ flow which produces a steady, stable shock wave.

Figure 6.9 shows instantaneous Q-criterion contours for hybrid RANS-LES of the 


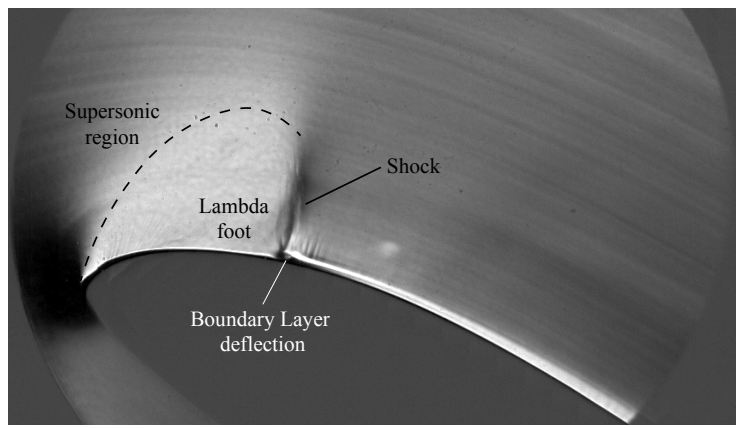

(a) Experimental schlieren image

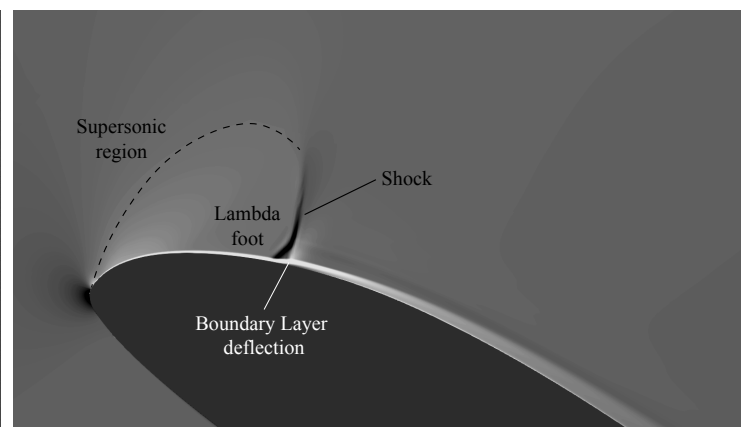

(b) CFD RANS-SA schlieren image $(d \rho / d y)$

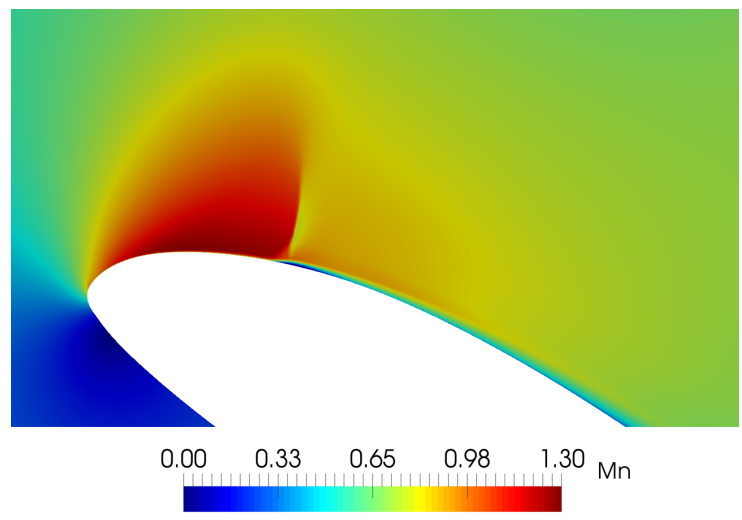

(c) CFD RANS-SA Mach number distribution

Fig. 6.8 Comparison of flow features between experimental and CFD results for $\alpha=23^{\circ}$

baseline flow. An iso-surface at $M=1$ is also shown displaying the boundary of the supersonic region. Flow at the leading edge of the aerofoil is in a laminar state as it initially accelerates over lip surface. The onset of transition to turbulence occurs upstream of the shock and SWBLI with transition complete at the SWBLI. This displays the transitional nature of the SWBLI.

Static pressure coefficient $\left(C_{P}\right)$ distribution over the intake lip is shown in Fig. 6.10 comparing CFD to experimental measurements. Results display a sharp decrease in $C_{P}$ at the leading edge of the upper aerofoil surface as a result of the severe flow acceleration over the lip. The surface pressure plateaus under the supersonic region, before abruptly increasing at the shock at $d S / L \approx 1$. This displays the severe adverse pressure gradient experienced by the boundary layer in the shock foot region. The observed distributions is typical of transonic flows. All CFD methods are able to reproduce this distribution well, although the the standard RANS-SA model predicts shock location $0.1 \mathrm{~L}$ upstream compared to experimental measurements. The modified SA model modified to account for the effects of acceleration and curvature (labelled 


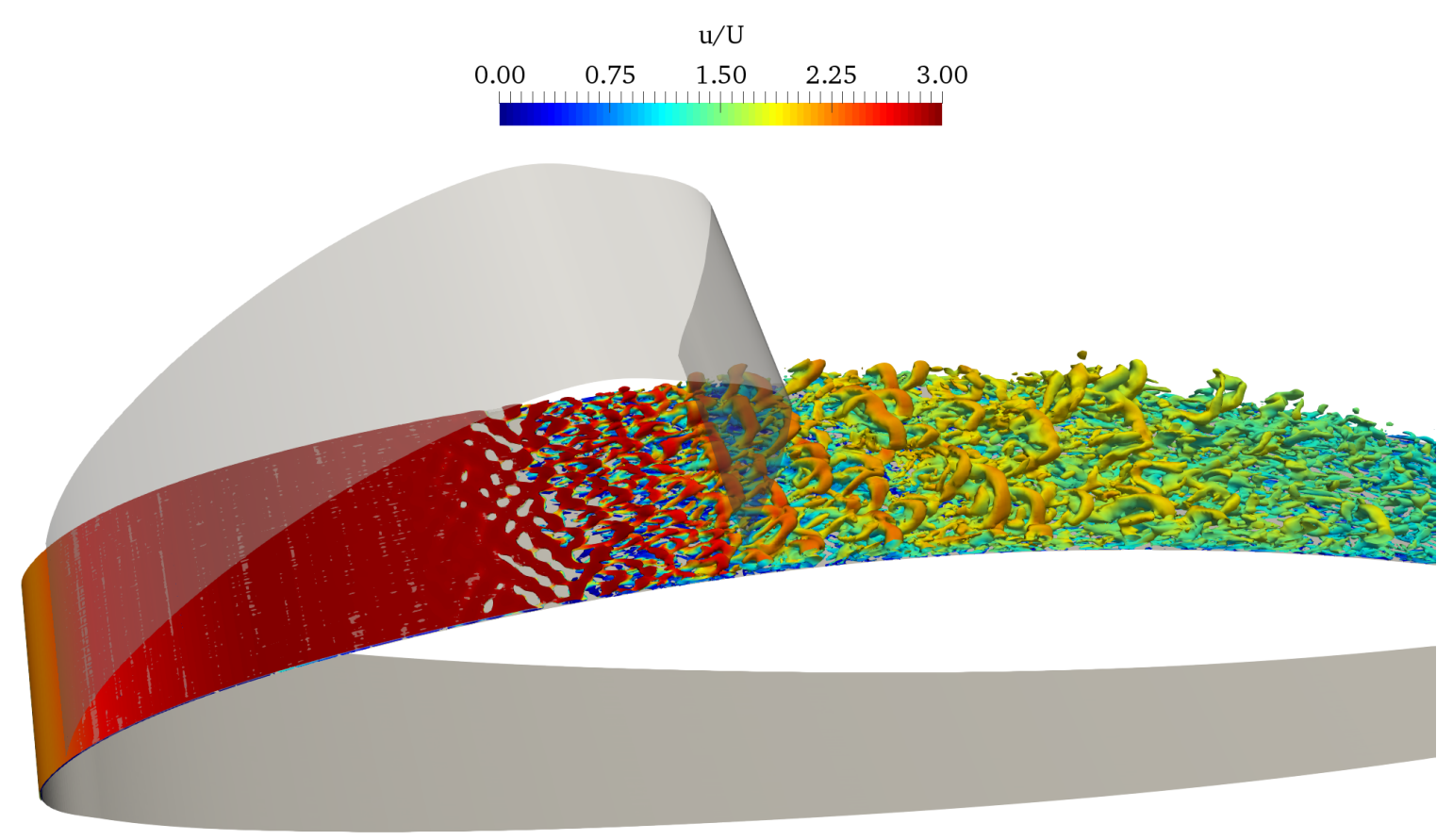

Fig. 6.9 Instantaneous contours of Q-criterion coloured by velocity for hybrid RANSLES at $\alpha=23^{\circ}$. Note the grey iso-surface showing the boundary of the supersonic region $(\mathrm{M}=1)$

RANS-SA Mod) provides a noticeable improvement to this, moving the shock further downstream, agreeing well with experimental measurements and hybrid RANS-LES. This is believed to be due to the modified SA model producing a thinner boundary layer upstream of the shock. This modifies the effective aerofoil shape, changing the local curvature here which moves the shock downstream and in line with experimental measurements. It can be noted that all predictions deviate from measurements at the immediate leading edge of the aerofoil $(d S / L<0.4)$. This is believed to be due to minor differences between the experimental and computational geometries that are highlighted in this highly accelerated region.

Figure 6.11 shows time-averaged velocity profiles normal to the aerofoil surface measured at 3 locations downstream of the shock and SWBLI. The flow here is significantly distorted, featuring large unsteady three dimensional vortical structures. While RANS is able to predict the correct boundary layer thickness here, the shape of the profiles do not agree well with measurements. Both the standard and modified RANS-SA models show a velocity deficit towards the aerofoil surface. Hybrid RANSLES on the other hand is able to give a much better prediction of the post SWBLI flow. The shape of the velocity profile agrees well with experimental measurements 


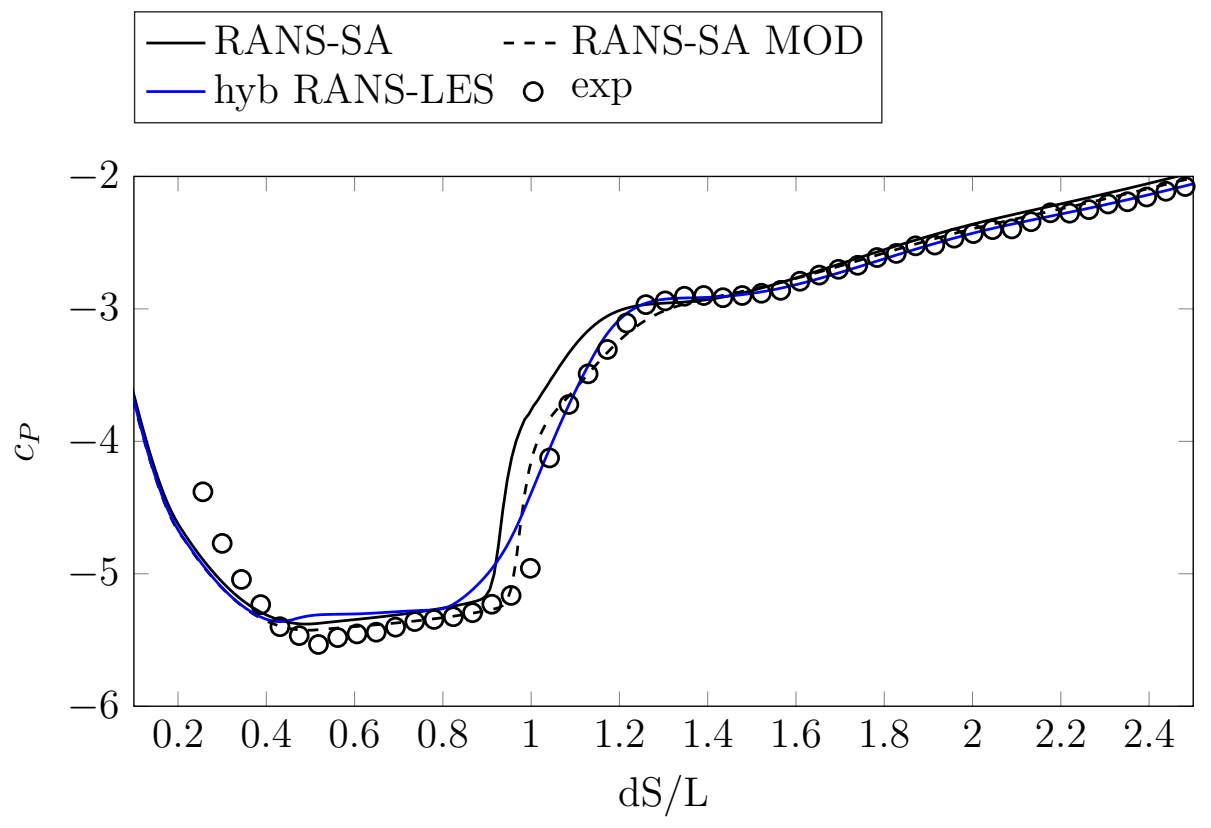

Fig. $6.10 C_{P}$ distribution over the intake lip for $\alpha=23^{\circ}$

at all 3 locations. This trend is also observed for the $\alpha=25^{\circ}$ case and is discussed further in the next section.

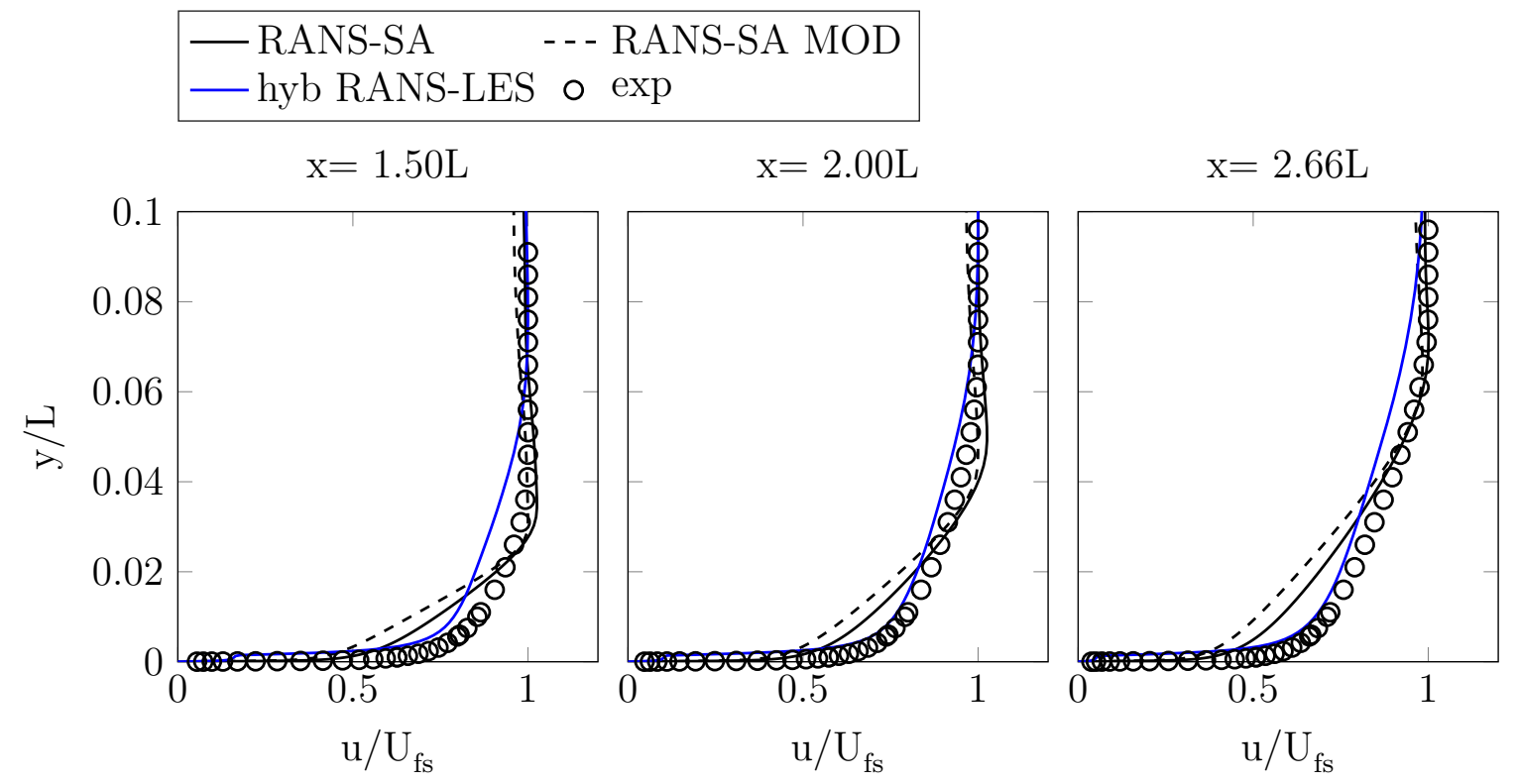

Fig. 6.11 Velocity profiles at 3 locations normal to the intake aerofoil surface for $\alpha=23^{\circ}$

Figure 6.12 shows the time-averaged skin friction coefficient $\left(C_{f}\right)$ over the intake 
lip at $\alpha=23^{\circ}$, defined,

$$
C_{f}=\frac{\tau_{w}}{\frac{1}{2} \rho_{\infty} U_{\infty}^{2}}
$$

where $\tau_{w}$ is the wall shear stress. $C_{f} \leq 0$ indicates flow separation. A summary of the time-averaged separation points, reattachments points and separation bubble size are given in table 6.1. Note that experimental measurements of $C_{f}$ are not available. RANS-SA and hybrid RANS-LES show $C_{f}$ peak at $d S=0.15 L$, where $\tau_{w}$ is highest due to the strong flow acceleration over the intake leading edge. Hybrid RANS-LES then shows a gradual $C_{f}$ decrease under the supersonic flow region going negative at $d S=0.72 L$ indicating flow separation here. Using hybrid RANS-LES, reattachment occurs at $d S=1.15 \mathrm{~L}$. Both the standard and modified RANS-SA models show $C_{f}$ plateau under the supersonic region, before sharply falling at the shock foot region. The standard and modified SA model predict flow separation at $d S=0.94 L$ and $d S=0.98 L$ respectively, the latter being due to the modified SA model giving a shock further downstream than the standard SA. As shown, Hybrid RANS-LES separates much earlier than the RANS models and as a result, predicts a separation bubble size almost double that of the RANS models.

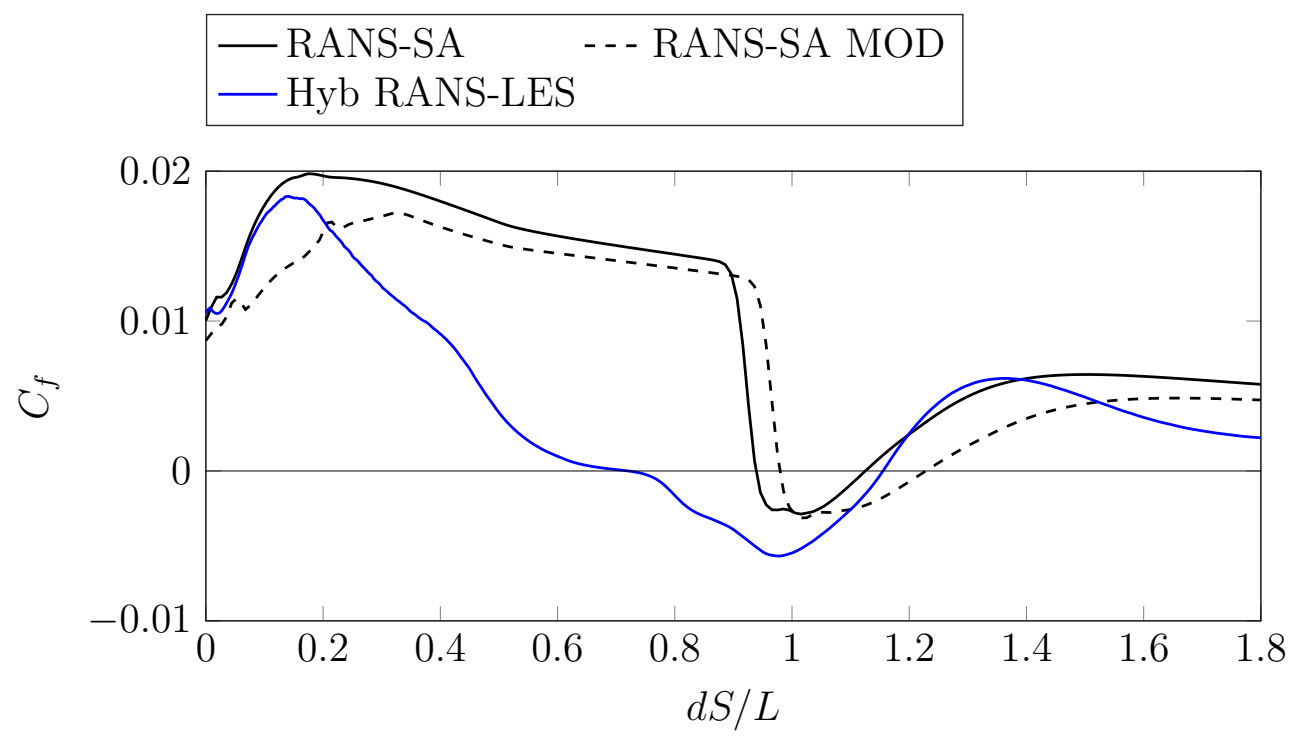

Fig. 6.12 Skin friction coefficient distribution over the intake aerofoil lip for $\alpha=23^{\circ}$

\subsection{2 $\alpha=25^{\circ}$ Case}

For $\alpha=25^{\circ}$, shock unsteadiness is observed. The shock is also stronger than in the baseline case at $M=1.4$. Figure 6.13 shows instantaneous $\mathrm{Q}$ contours from LES of 


\begin{tabular}{|l|l|l|l|}
\hline & $\begin{array}{l}\text { Separation } \\
\text { Point }(\mathrm{dS} / \mathrm{L})\end{array}$ & $\begin{array}{l}\text { Reattachment } \\
\text { point }(\mathrm{dS} / \mathrm{L})\end{array}$ & $\begin{array}{l}\text { Bubble } \\
\text { size }(\mathrm{dS} / \mathrm{L})\end{array}$ \\
\hline RANS-SA & 0.94 & 1.14 & 0.20 \\
\hline RANS-SA Mod & 0.98 & 1.23 & 0.25 \\
\hline Hybrid RANS-LES & 0.72 & 1.15 & 0.43 \\
\hline
\end{tabular}

Table 6.1 Separation, reattachment and bubble size over the intake aerofoil lip for $\alpha=23^{\circ}$

the $\alpha=25^{\circ}$ case. Finer flow structures can be noted compared to hybrid RANS-LES shown in Fig. 6.9 due to the increased grid resolution for LES. As with the baseline case, the flow is laminar over the aerofoil leading edge, undergoing shock induced transition and breaking down to fully turbulent flow downstream of the SWBLI.

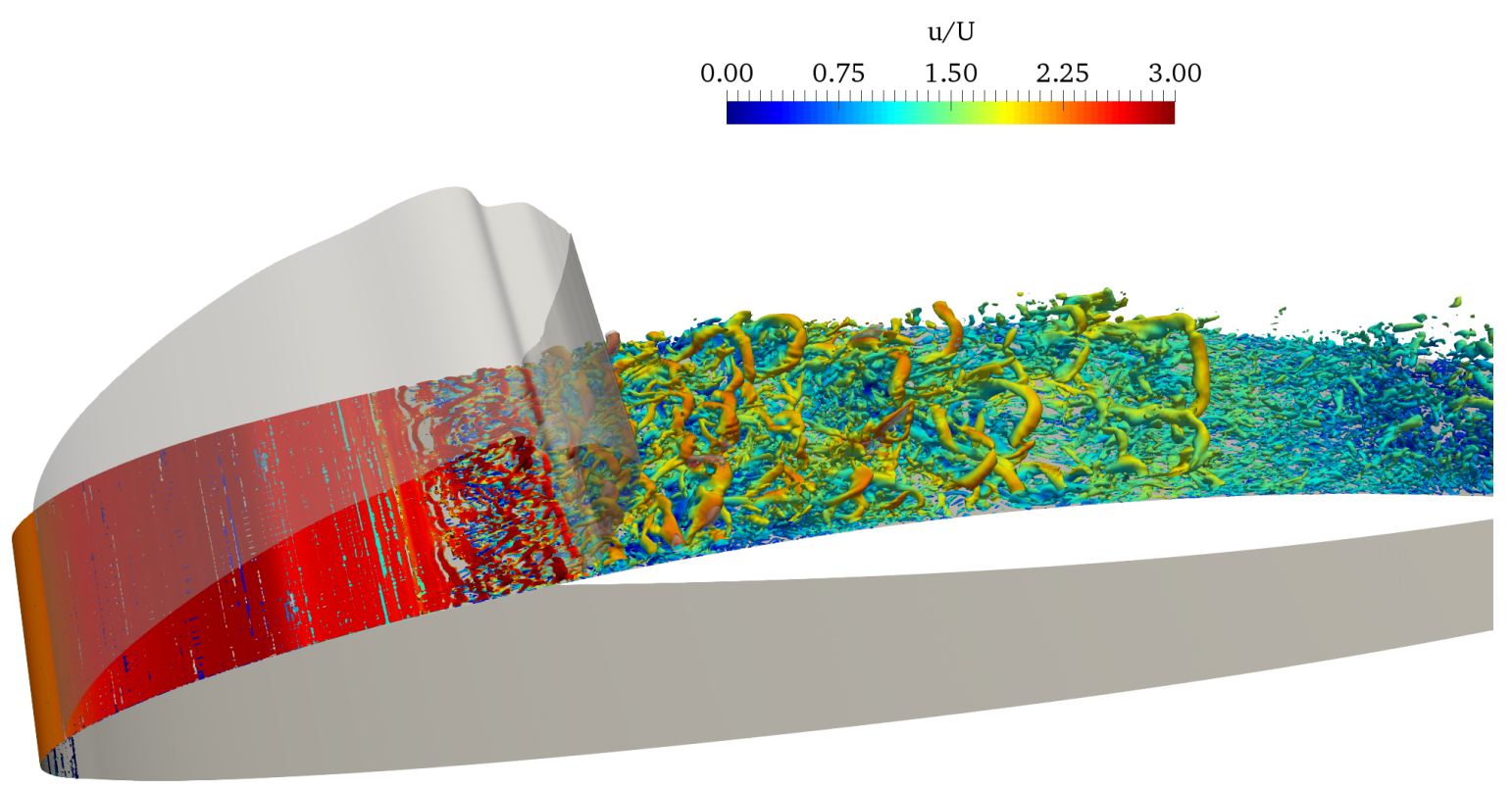

Fig. 6.13 Instantaneous contours of Q-criterion coloured by velocity for LES at $\alpha=25^{\circ}$. Note the grey iso-surface showing the boundary of the supersonic region $(\mathrm{M}=1)$

Figure 6.14 compares the classical Jameson shock sensor and the improved Ducros sensor on the solver smoothing field used for hybrid RANS-LES and LES simulations of the intake rig. Note that the Jameson sensor field is only computed for visualisation purposes, and the Ducros sensor used for actual simulations. As was seen for the transonic hump, the Jameson sensor has a high value in regions of turbulent fluctuations as well as the shock, while the Ducros sensor is able to isolate this and therefore 
preserve the solution accuracy.

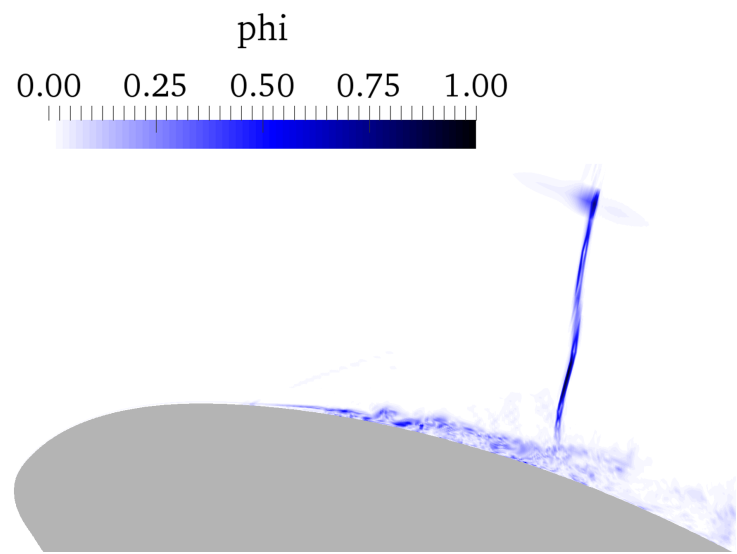

(a) Jameson sensor

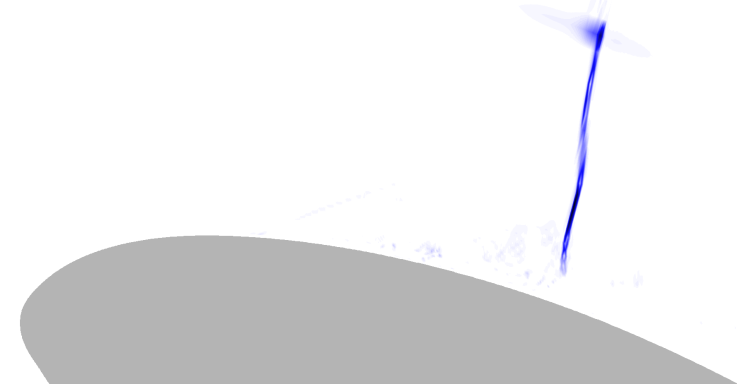

(b) Ducros sensor

Fig. 6.14 Comparisson of the classical Jameson shock sensor and the improved Ducros sensor

Figure 6.15 shows the time-averaged $C_{P}$ distribution over the lip surface for the $\alpha=25^{\circ}$ from CFD and experimental measurements. The pressure recovery in the shock foot region is spread over a longer distance compared to the baseline case due to the shock unsteadiness. The shock foot was roughly measured by experiments to oscillate between $d S / L \approx 0.90$ to $d S / L \approx 1.35$ giving an amplitude of $d S / L=0.45$ (corresponding to approximately $25 \mathrm{~mm}$ ). The steady RANS simulations are unable to account for this and instead show a fixed, well defined shock location. Furthermore, as with the baseline case, the standard RANS-SA model predicts a shock too far upstream compared to measurements. This is more pronounced for $\alpha=25^{\circ}$. The modified RANS-SA model does provide an improvement here, moving the shock closer towards measurements. This is again believed to be influenced by the modified RANSSA producing a thinner boundary layer upstream of the SWBLI, however it is still fundamentally unable to capture the time-averaged shock smearing. Figure 6.16 shows time-averaged contours of Mach number over the aerofoil lip region from the CFD methods. The smearing of the shock captured by hybrid RANS-LES and LES is visible here, while RANS predicts a fixed shock and SWBLI location.

As a result, hybrid RANS-LES and LES simulations agree very well with experimental $C_{P}$ measurements. Both hybrid RANS-LES and LES are able to capture the smearing of the shock as well as the correct shock location and shock oscillation amplitude.

The failure of RANS to predict mean surface pressures due to the high level of unsteadiness in the shock system is a trend observed by many authors [8, 43, 47-49, 51] 


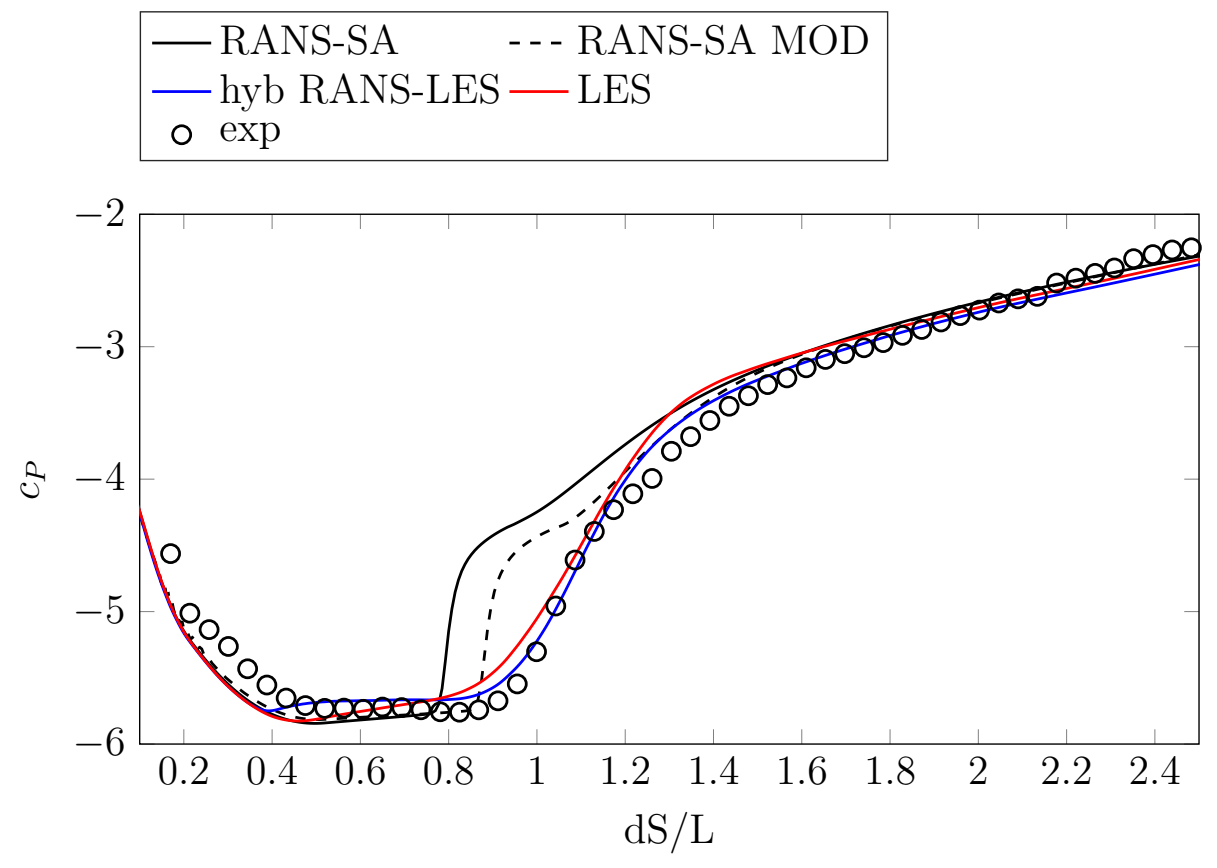

Fig. $6.15 C_{P}$ distribution over the intake lip for $\alpha=25^{\circ}$

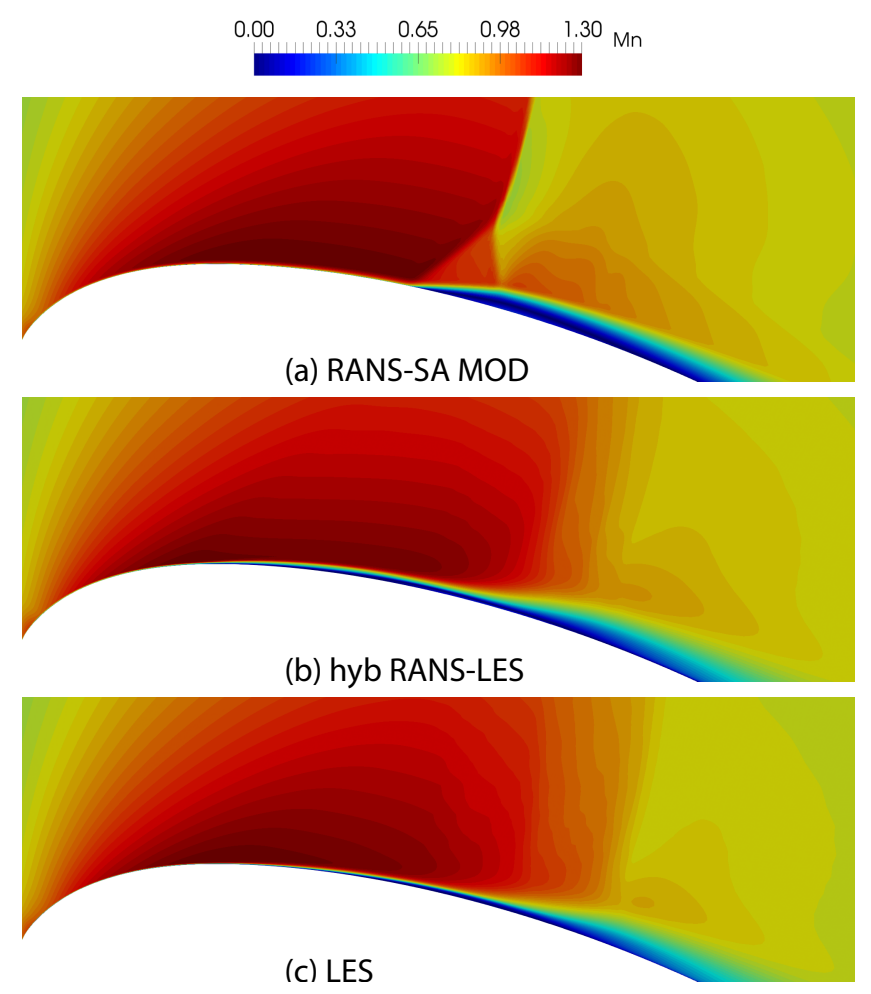

Fig. 6.16 Time-averaged contours of Mach number over the aerofoil lip region for $\alpha=25^{\circ}$ 
as discussed in the literature review. As summarised by Dolling [43], RANS computations of SWBLIs are satisfactory for weak interactions (without flow separation), but poor agreement is seen for strong interactions. This is attributed to low frequency unsteadiness which can be a dominant feature of such flows [43]. This same trend is also observed here, when comparing between the $\alpha=23^{\circ}$ and $\alpha=25^{\circ}$ cases.

Fig. 6.17 shows a velocity profile normal to the aerofoil surface measured at one location downstream of the shock and SWBLI for $\alpha=25^{\circ}$. Again, we see that both RANS methods are unable to produce the correct profile shape here, showing a velocity deficit towards the aerofoil surface. Hybrid RANS-LES and LES instead perform very well. We can attribute this to the highly distorted three dimensional turbulent flow here that RANS cannot reproduce. Further, we can note that hybrid RANS-LES performs very close to LES in predicting these first order moments despite costing significantly less than LES.

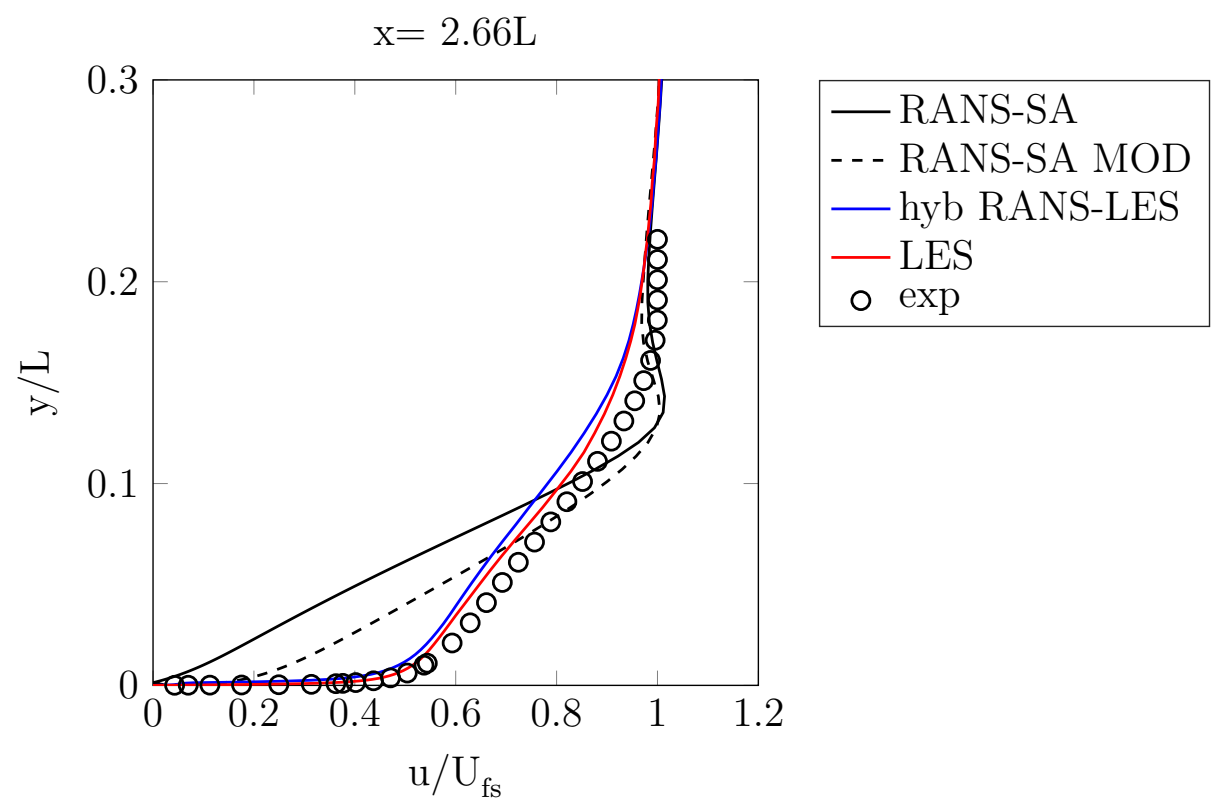

Fig. 6.17 Velocity profiles at one location normal to the intake aerofoil surface for $\alpha=25^{\circ}$

We can further compare the performance of the CFD methods at five equally spaced locations over the lip from $d S / L=0.5$ to 2.5 , although experimental data is not available here. Figure 6.18 shows velocity and turbulence intensity $(\mathrm{Ti})$ profiles at these locations. $T i$ is defined,

$$
T i=\sqrt{\frac{1}{3} \frac{\overline{u^{\prime} u^{\prime}}+\overline{v^{\prime} v^{\prime}}+\overline{w^{\prime} w^{\prime}}}{U_{r e f}^{2}}}
$$


where the primes $\left({ }^{\prime}\right)$ denote the fluctuating part of the velocity respectively. Note that $T i$ is only shown for the eddy resolving hybrid RANS-LES and LES simulations. We can see in Fig. 6.18a at $d S=0.5 \mathrm{~L}$ and $d S=1.0 \mathrm{~L}$ (which are upstream of the shock and SWBLI) that the standard RANS-SA model predicts an oncoming boundary layer that is thicker compared to hybrid RANS-LES and LES. This results in a shock location prediction upstream of measurements as seen earlier. We can also see at $d S=1.0 L$ that the modified RANS-SA model thins this boundary layer. The remaining velocity profiles display the trend previously observed, whereby the RANS velocity profile shapes are in disagreement with hybrid RANS-LES and LES.

For $T i$, we see hybrid RANS-LES in good agreement with LES, further demonstrating hybrid RANS-LES suitability to this flow. Only at the first location $d S=0.5 L$ is the peak $T i$ over-predicted for hybrid RANS-LES compared to LES. At this location, close to the leading edge of the aerofoil, the boundary layer is very thin and so the fixed RANS layer used occupies a large amount of the boundary layer here. This, in addition to the RANS-LES interface zone (the so-called 'grey area') can be considered responsible for the discrepancies here. Ti however recovers as the boundary layer grows, giving good agreement with LES at the remaining locations.

Figure 6.19 shows the time-averaged skin friction coefficient $\left(C_{f}\right)$ over the intake lip at $\alpha=25^{\circ}$. A summary of the time-averaged separation points, reattachments points and separation bubble size are given in table 6.2. Since experimental measurements of $C_{f}$ are not available, comparisons are made with the LES results. LES shows $C_{f}$ peak at $d S=0.15 L$, where the $\tau_{w}$ is highest due to the strong flow acceleration over the intake leading edge. $C_{f}$ then gradually decreases under the supersonic flow region going negative at $d S=0.76 \mathrm{~L}$ indicating flow separation here. LES reattachment occurs at $d S=1.26 L$. Both the standard and modified RANS-SA models over-predict peak $C_{f}$. They also show $C_{f}$ plateau under the supersonic region, before sharply falling at the shock foot region, as was seen for the $\alpha=23^{\circ}$ case. The standard and modified SA model predict flow separation at $d S=0.80 L$ and $d S=0.88 L$ respectively, the latter being due to the modified SA model giving a shock further downstream than the standard SA. Both RANS models over-predict the separation bubble size, with the standard RANS-SA model exaggerating this by $50 \%$ compared to LES. Hybrid RANSLES $C_{f}$ prediction displays characteristics of both RANS and LES. Up to $d S \approx 0.2 L$, hybrid RANS-LES performs exactly as RANS, over predicting peak $C_{f}$. As discussed earlier, this is where the boundary layer is very thin and the RANS layer dominates. However downstream of this, hybrid RANS-LES agrees reasonably well with LES, giving flow separation and reattachment locations of $d S=0.75 L$ and $d S=1.31 L$. 


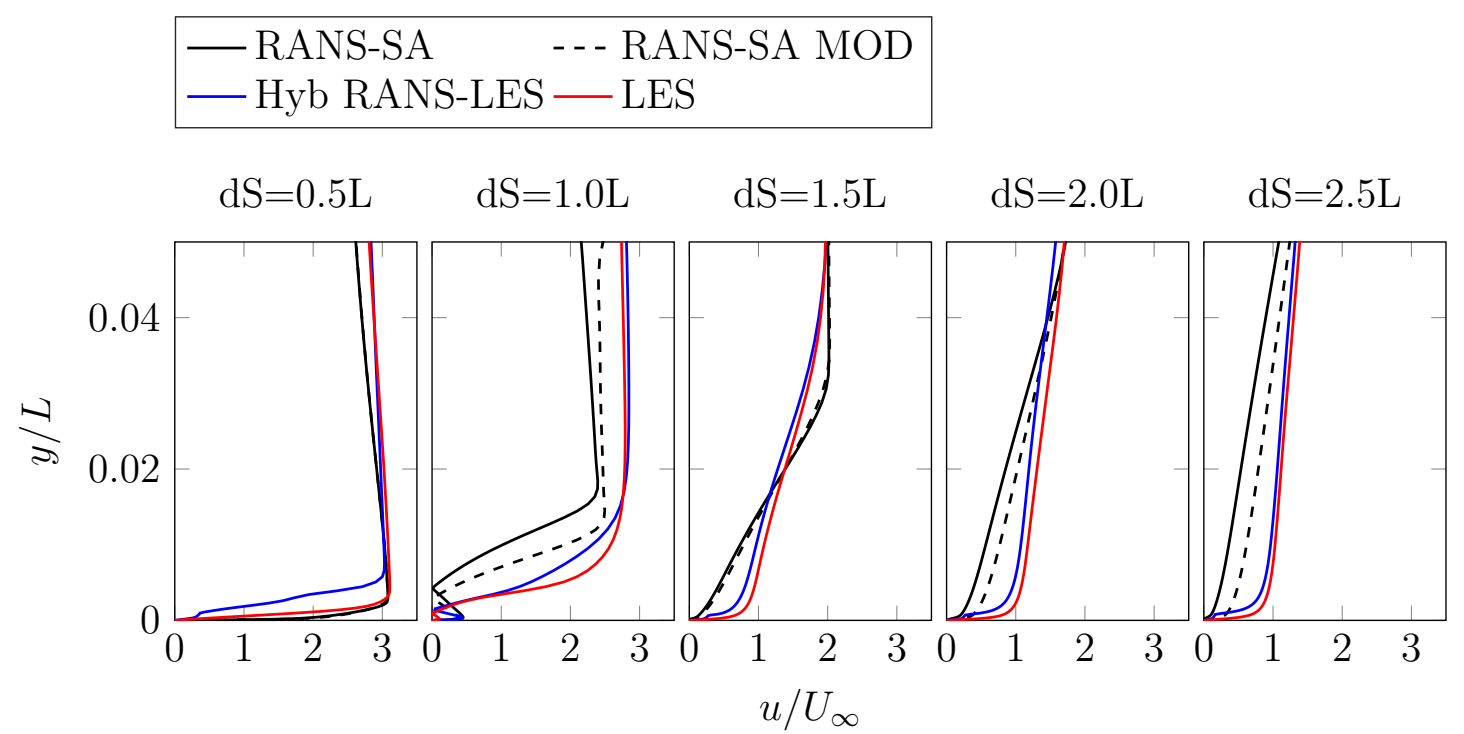

(a) Velocity profiles

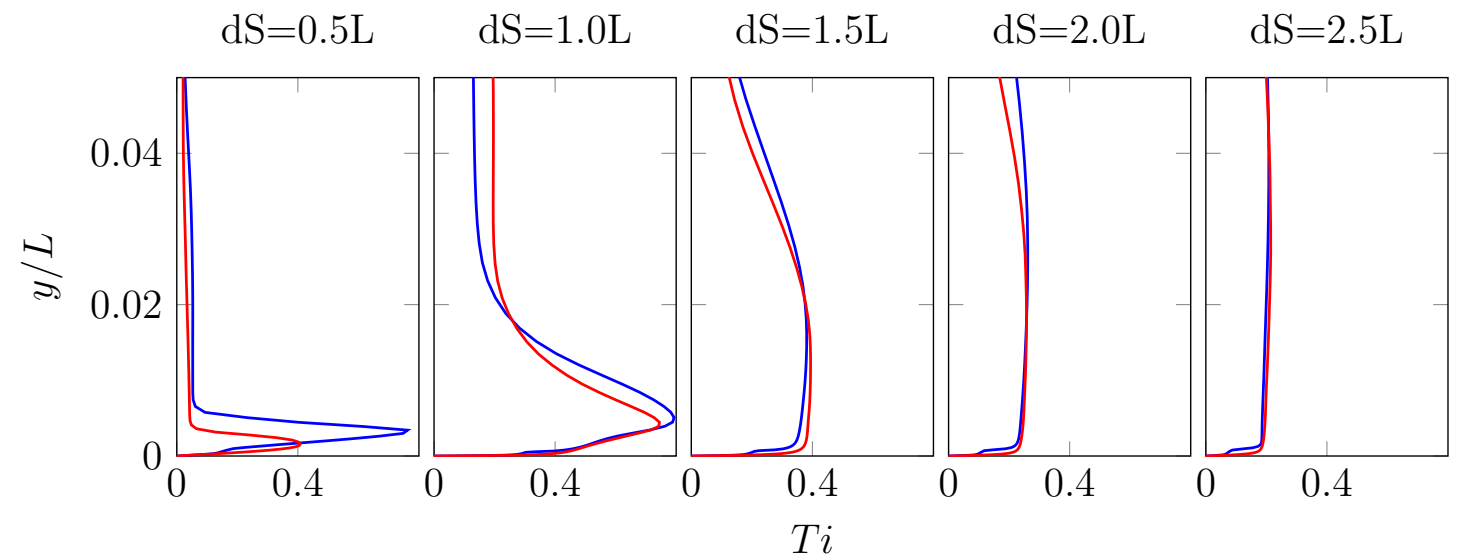

(b) Turbulence intensity profiles

Fig. 6.18 Velocity and turbulence intensity profiles at 5 locations over the intake aerofoil lip for $\alpha=25^{\circ}$

\begin{tabular}{|l|l|l|l|}
\hline & $\begin{array}{l}\text { Separation } \\
\text { Point }(\mathrm{dS} / \mathrm{L})\end{array}$ & $\begin{array}{l}\text { Reattachment } \\
\text { point }(\mathrm{dS} / \mathrm{L})\end{array}$ & $\begin{array}{l}\text { Bubble } \\
\text { size }(\mathrm{dS} / \mathrm{L})\end{array}$ \\
\hline RANS-SA & 0.80 & 1.55 & 0.75 \\
\hline RANS-SA Mod & 0.88 & 1.51 & 0.63 \\
\hline Hybrid RANS-LES & 0.75 & 1.31 & 0.56 \\
\hline LES & 0.76 & 1.26 & 0.50 \\
\hline
\end{tabular}

Table 6.2 Separation, reattachment and bubble size over the intake aerofoil lip for $\alpha=25^{\circ}$ 


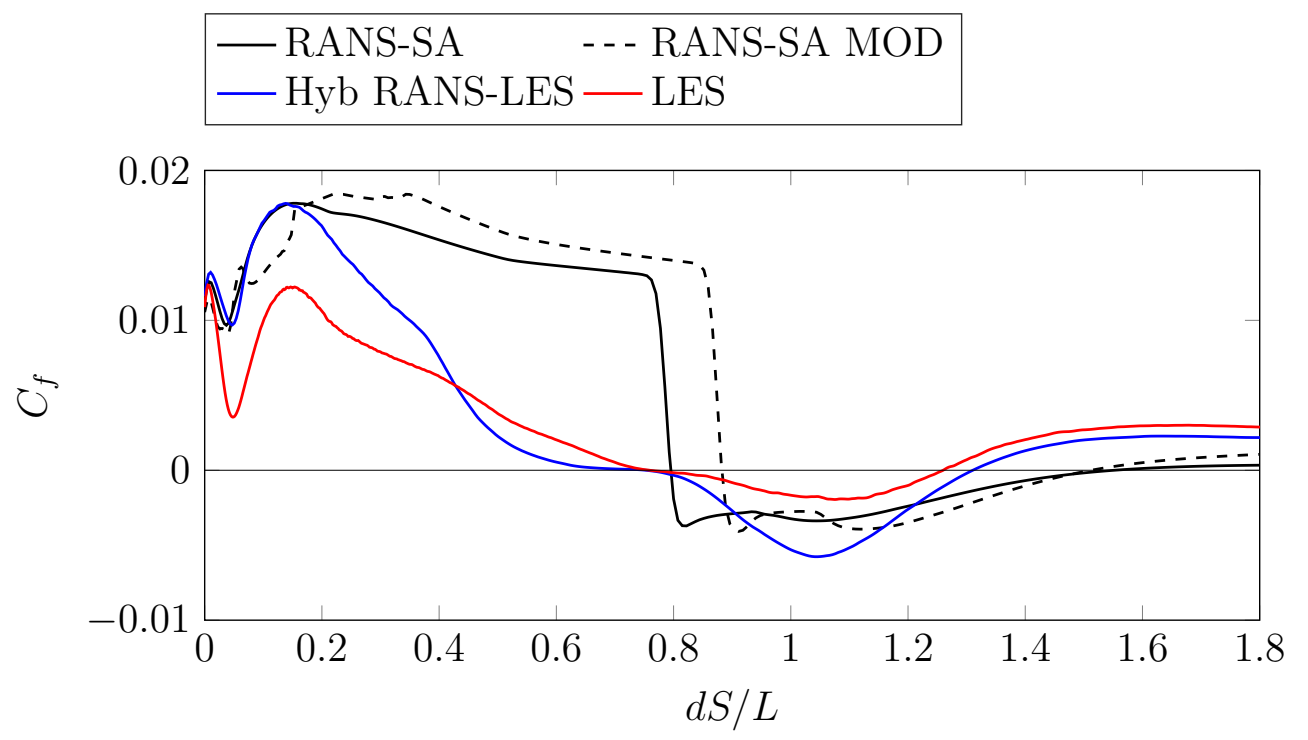

Fig. 6.19 Skin friction coefficient distribution over the intake aerofoil lip for $\alpha=25^{\circ}$

The shock unsteadiness for the $\alpha=25^{\circ}$ case presents a challenge for unsteady eddy resolving simulations as these must be run for a sufficiently long simulation time in order to acquire converged time-averaged flow statistics. This is particularly expensive for wall resolved LES as the fine grids used mean the timestep size must also reduced to maintain numerical stability. In this work, LES of the $\alpha=25^{\circ}$ case could only be run for $7 T^{*}$. Due to its reduced computational cost, Hybrid RANS-LES on the other hand was run for $12 T^{*}$. Hybrid RANS-LES was calculated to cost approximately $20 \%$ of LES for the same simulation time and as shown, performs very close to LES for this flow. This displays the significant benefits hybrid RANS-LES can offer. The close results of hybrid RANS-LES to LES implies that the precise resolution of the near wall behaviour is not vital to the accurate prediction of this flow, with the behaviour of the shock, shock dynamics, SWBLI and post shock flow of more importance. This agrees with Piomelli's review [52] that hybrid RANS-LES methods are most effective in flow conditions that facilitate the rapid amplification of instabilities where, as shown, SWBLIs are suited.

The increased fidelity of LES does allow additional study of flow physics. Figure 6.20 shows Instantaneous contours of Q-criterion coloured by velocity using LES for $\alpha=25^{\circ}$ at 3 time instances corresponding to the shock in its most upstream location (Figure 6.20a), mid period location (Figure 6.20b) and most downstream location (Figure 6.20c). $x_{t}$ and $x_{s}$ mark the onset of transition and shock location respectively. As shown, the transition length $\left(x_{s}-x_{t}\right)$ varies with shock location. The onset of transition is coupled to a secondary compression wave shown in figure 6.21. This 
is captured by LES as shown in figure 6.21a and qualitatively compares well with experimental schlieren images. The compression wave is believed to form from the abrupt increase in boundary layer thickness at the point of transition. This travels through the supersonic region towards the main shock to which it is also coupled. As the shock oscillates, this influences the location of $x_{t}$ directly. Future experimental data is planned to investigate this further, such as characterisation of the upstream boundary layer using infra-red techniques. Nevertheless, results display LES's ability to also reproduce secondary flow features present in this complex flow.

\subsection{Conclusion}

Relaminarisation and curvature corrections to the RANS SA model provide a significant improvement in prediction of shock location and lip surface pressure for intakes at incidence. However, RANS fails to reproduce the post shock interaction flow well, giving incorrect shape of the flow distortion. Further, if the flow exhibits shock unsteadiness as seen at higher angles of incidence, RANS is inherently unable to capture the time-averaged smearing of the shock and related unsteady flow features. Accurately predicting this post interaction flow is crucial for aero-engine intake design, as it is this flow that would be propagating towards the engine fan face.

LES and hybrid RANS-LES predict the shock location and SWBLI well, with the downstream flow distortion also in very good agreement with experimental measurements. LES and hybrid RANS-LES are able to reproduce the time-averaged smearing of the shock which RANS cannot. LES is also able to reproduce secondary flow features such as compression waves that accompany the main shock. However, shock oscillations present a particular challenge for costly LES, requiring long simulation time to obtain time-averaged flow statistics. Hybrid RANS-LES offers a significant saving in computational cost, costing approximately $20 \%$ of LES. Hybrid RANS-LES is therefore recommended for simulations of this flow type and looks promising for future use in industry. 
(a)

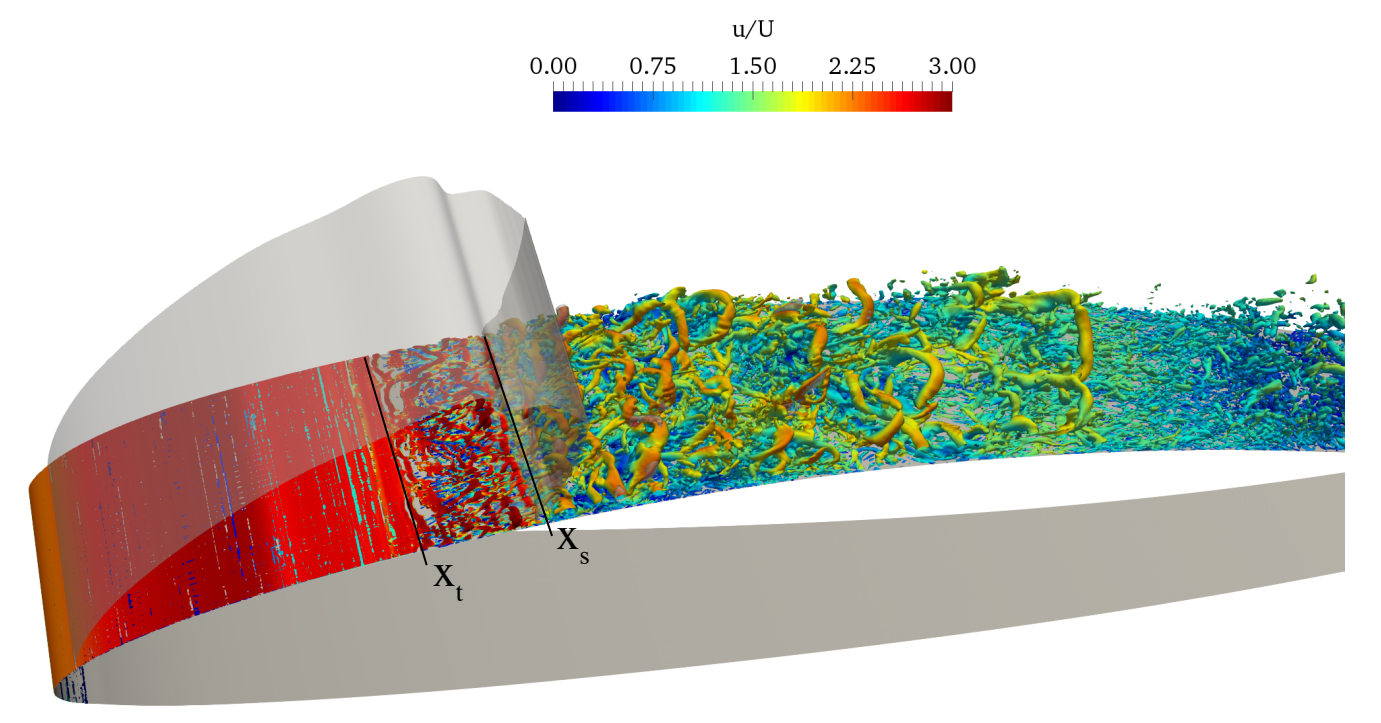

(b)

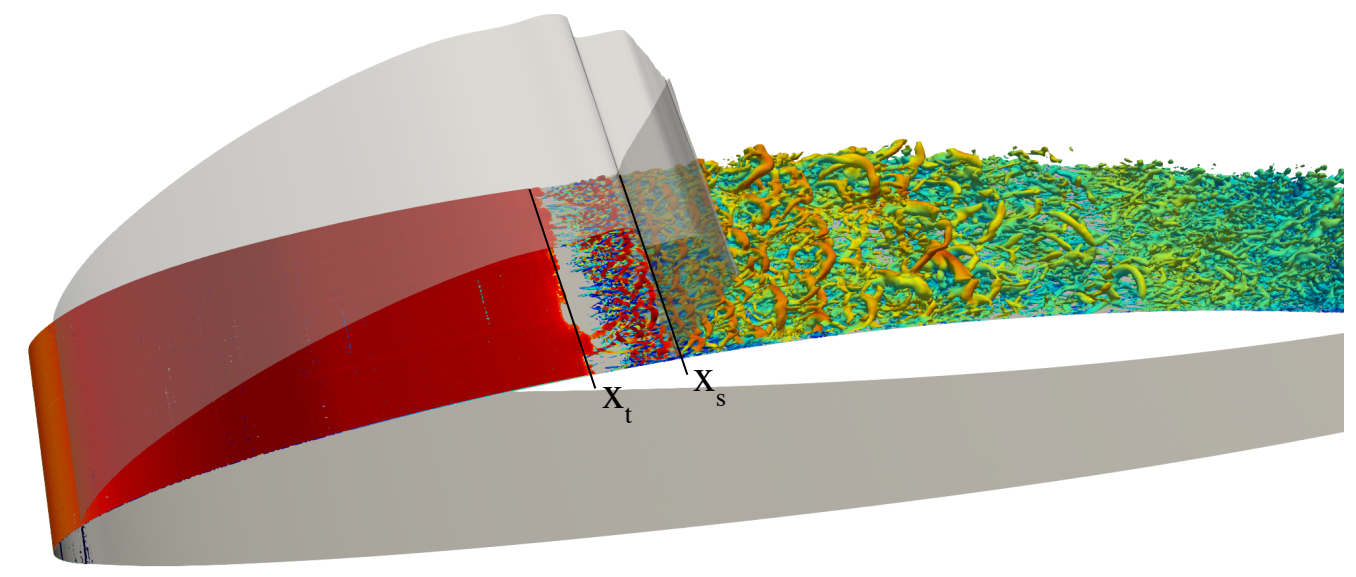

(c)

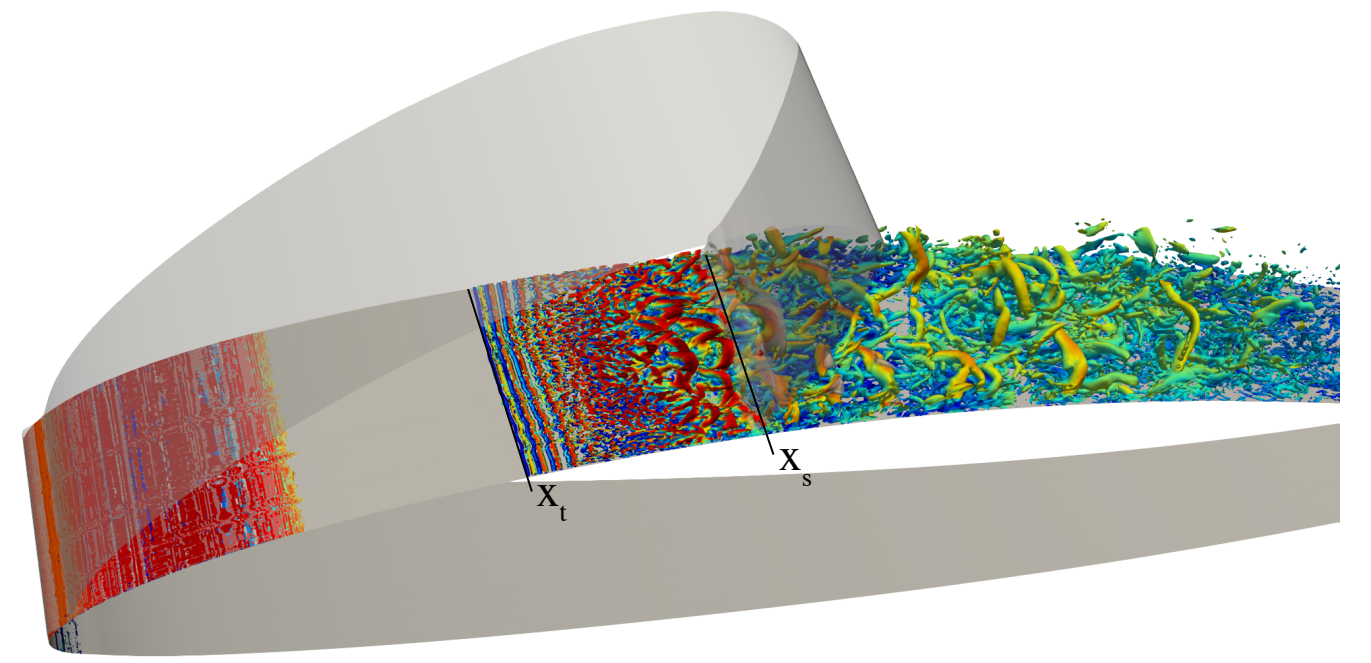

Fig. 6.20 Instantaneous contours of Q-criterion coloured by velocity using LES for $\alpha=25^{\circ}$ at 3 time instances. Note the grey iso-surface showing the boundary of the supersonic region $(\mathrm{M}=1) . x_{t}$ and $x_{s}$ mark the onset of transition and shock location respectively 


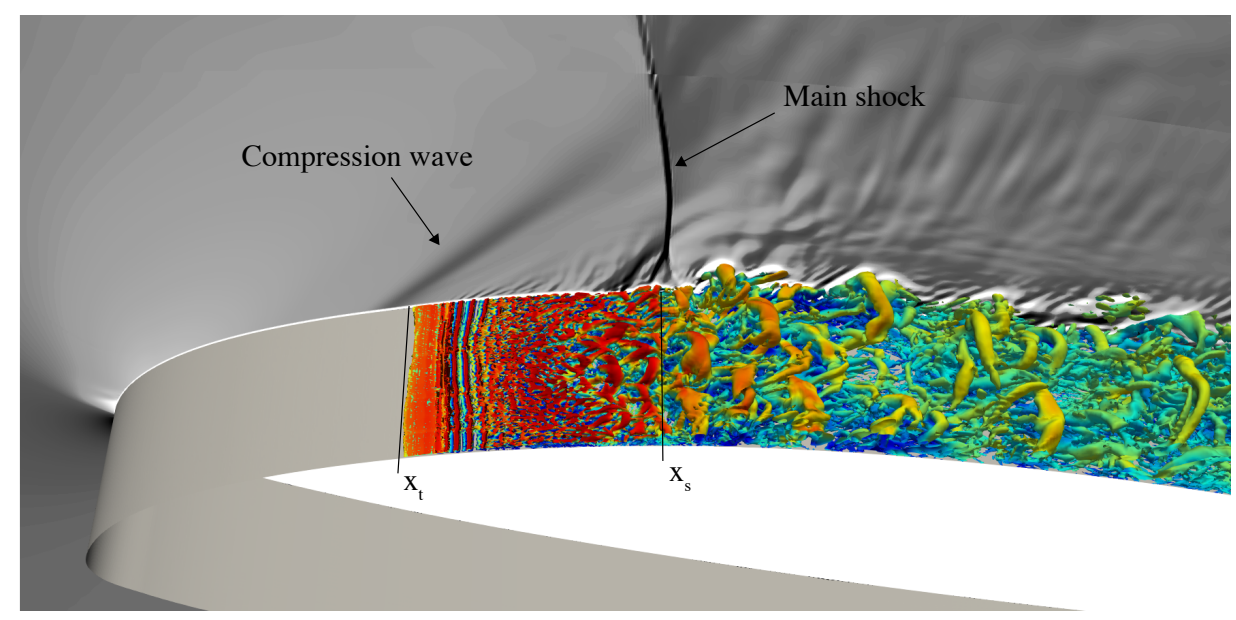

(a) Instantaneous contours of Q-criterion coloured by velocity using LES with a schlieren-like plane of $d \rho / d y$ shown at the spanwise extent. $x_{t}$ and $x_{s}$ mark the onset of transition and shock location respectively.

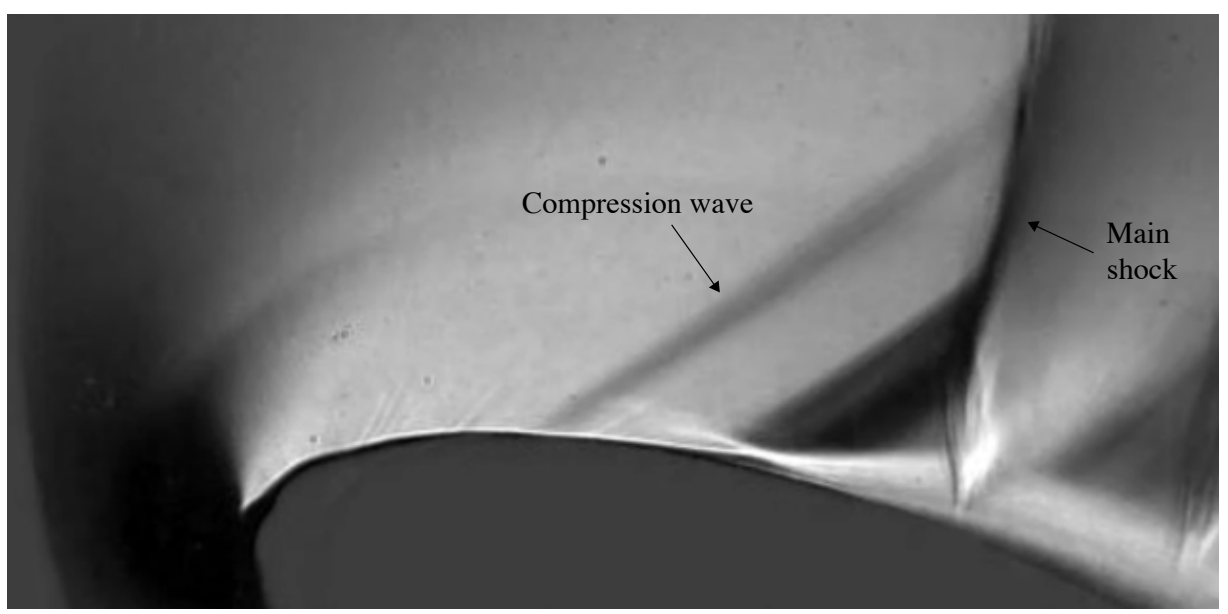

(b) Experimental schlieren image

Fig. 6.21 Comparisson of secondary flow features between CFD and exprimental schlieren image for $\alpha=25^{\circ}$ 



\section{Chapter 7}

\section{Concluding Remarks and Recommendations for Future Work}

\subsection{Concluding Remarks}

Intakes operating at high incidence present an interesting and challenging case for numerical modelling. The coupling of flow physics here, including rapid flow accelerations, SWBLIs and flow separation, mean numerical methods must be sensitive to a wide range of flow physics in order to predict the flow correctly. This project aimed to improve the modelling of this complex flow and provide a heirarchy of predictive strategies using RANS, LES and hybrid RANS-LES, comparing to new intake rig measurements.

Modifications to the one-equation Spalart-Allmaras (SA) RANS turbulence model to account for the effects of relaminarisation and curvature gave a noticeable improvement in surface pressure predictions and shock location, even for mild accelerations. The correct prediction of shock location is important as found on the transonic hump case, as this can significantly affect the flow downstream; especially where shock induced separation occurs. For simulations of the intake rig, RANS compared well to the baseline steady flow case. However, RANS models failed to reproduce the post shock interaction flow well, giving incorrect shape of downstream velocity profiles. This is a known defect of RANS, where the RANS formulation is fundamentally unable to account for highly unsteady three-dimensional turbulent motions here. However, the cheapness of RANS means that it will continue to be a popular tool in industry. Results in this work show that RANS predicts SWBLI flows well for cases where the interaction is steady and the separation is small, and is therefore only recommended for such cases. 
For eddy resolving simulations, LES is shown to be a superior modelling technique for unsteady turbulent flows. Coupled with the $\sigma$-SGS model, LES is shown to be the best predictive tool used in this work for separated flows and unsteady SWBLI predictions. LES is also shown to be able to reproduce secondary transonic flow features such as transition induced compression waves that can enable study of these phenomena in detail. Its natural ability to resolved a wide range of turbulent scales in all parts of the flow mean that it can be considered the most reliable simulation method.

However, shock unsteadiness that is present in most shocked flows of interest presents a particular challenge for costly LES, requiring long simulation time to obtain time averaged flow statistics. This is where hybrid RANS-LES offers significant advantages. Hybrid RANS-LES is shown to perform close to LES for separated flows and unsteady SWBLI predictions, despite costing approximately $20 \%$ of LES. LES and hybrid RANS-LES are both able to reproduce the time averaged smearing of the shock as well as downstream flow distortions, which RANS cannot. The use of the Ducros shock sensor is also recommended for use with eddy resolving simulations, where the superior shock detection helps to preserve numerical accuracy. The close results of hybrid RANS-LES to LES implies that the precise resolution of the near wall behaviour is not vital to the accurate prediction of SWBLI flow, with the behaviour of the shock, shock dynamics, SWBLI and post shock flow of more importance. Specifically, hybrid RANS-LES methods are most effective in flow conditions that facilitate the rapid amplification of instabilities where, as shown, SWBLIs are suited. As a result, hybrid RANS-LES is the recommended simulation method for aero-engine intakes at incidence, and looks promising for future use in industry.

\subsection{Recommendations for Future Work}

The novel experimental rig simulated in this work presents many interesting opportunities for future work. New experimental measurements for the $\alpha=23^{\circ}$ and $\alpha=25^{\circ}$ are still being made, and so further validations with the simulation data already obtained in this project will be possible. Characterisation of the upstream boundary layer in particular will be interesting to explore.

It would also be interesting to explore the effects of a fully turbulent boundary layer on the SWBLI by numerically tripping this at the leading edge. This would lead to a turbulent SWBLI, with a boundary layer that may be more resilient to separation. This could be extended to the exploring the effects of lip surface roughness on the 
intake walls to represent acoustic liners that are present on real intakes.

The investigation of the effects of a fan downstream of the lip is also recommended for future work. In a real intake, the presence of the fan can significantly alter the flow. This includes suppression of the separation bubble or a redistribution of the mass flow. The flow distortion experienced by a fan downstream of SWBLI will also be interesting to study. A simple low order method (such as a bodyforce model) is recommended for simulating a fan in the upper channel of the intake rig. Coupled with LES or hybrid RANS-LES, this would provide a very interesting case for research.

The rig also allows varying shapes of the lip profile to be measured. These are also currently being experimentally tested. Since future intakes are moving towards thinner and shorter designs, the effects of varying the lip shape and profile on the flow and SWBLI is a topic that many are interested in. The good performance of LES and hybrid RANS-LES demonstrated in the work gives confidence in using these for other lip shapes, without the immediate need for experimental validation data.

With regards to numerical simulation methods, further zonalisation of the hybrid RANS-LES framework would be worth investigating. Figure 7.1 shows recommended zonalisation strategies, whereby the downstream extent of the wind tunnel and the intake aerofoil underside can be modelled using RANS, while the intake lip region can be wall resolved LES or hybrid RANS-LES. This would allow a significant saving in computational cost, and if successful, could be very useful for full 3D intake simulations. Other non-zonal hybrid RANS-LES methods such as Delayed Detached Eddy Simulation (DDES) could also be explored.

(a)

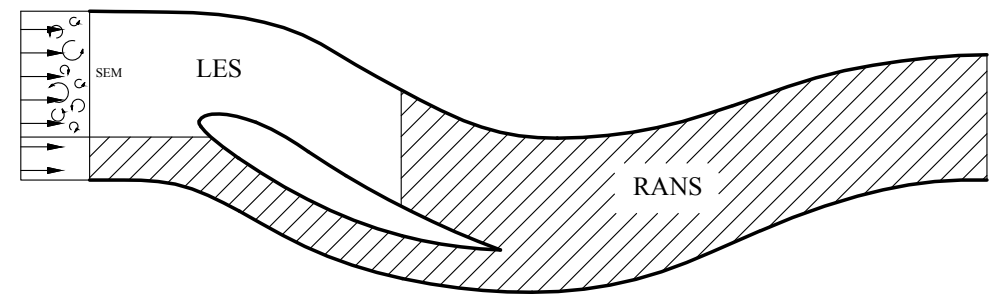

(b)

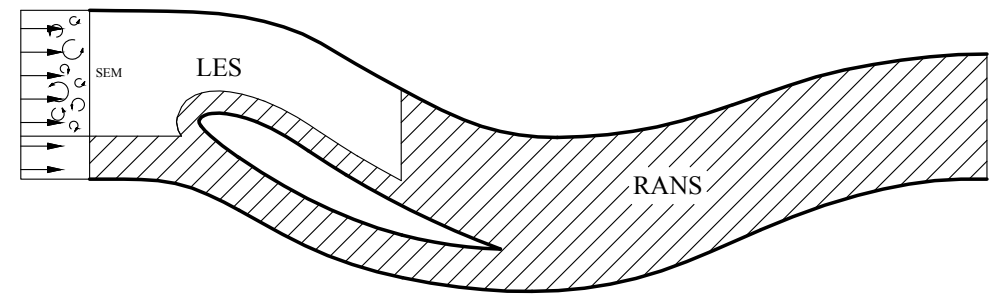

Fig. 7.1 Recommended zonalisation strategies for future intake rig simulations

Also shown in figure 7.1 is freestream turbulence at the wind tunnel inflow. Although turbulence for the rig was measured to be low, its effect on transition over the 
lip surface would be interesting for future work as real aero-engine intake experience freestream turbulence during operation. This may have the effect of transitioning the boundary layer upstream of the shock, leading to a turbulent SWBLI that is more resilient to separation. The Synthetic Eddy Method (SEM) to generate inflow turbulence has recently been implemented into the HYDRA CFD code, and so its use here would be recommended.

In addition to this, extending the relaminarisation modification used in this work to other RANS turbulence models is recommended. The modification noticeably improved predictions for high and low accelerations, and could provide better predictive ability for a wide variety of flows.

The final recommendation for future work would be to extend the CFD methods explored in this project to a 3D intake geometry at incidence. As explored in the literature review, curved 3D lip profiles have been experimentally and computationally tested, although this is for pure $90^{\circ}$ crosswind flow with no SWBLI. Hybrid RANS-LES would be recommended for simulation here, which could ultimately enable improved predictions for real intakes. 


\section{References}

[1] U. R. Oriji and P. G. Tucker. Modular Turbulence Modeling Applied to an Engine Intake. Journal of Turbomachinery, 136(5):051004, sep 2013.

[2] U. R. Oriji and P. G. Tucker. Hybrid RANS/ILES For Aero Engine Intake. In Proceedings of the ASME Turbo Expo 2014, pages 1-15, 2014.

[3] N. R. Vadlamani and P. G. Tucker. Eddy resolving simulations of intake under crosswinds. In Proceedings of 11th Direct and Large eddy simulation, pages 3-4, Pisa, Italy, 2017.

[4] C. A. Hall and T. P. Hynes. Nacelle Interaction with Natural Wind Before Takeoff. Journal of Propulsion and Power, 21(5), 2005.

[5] C. a. Hall and T. P. Hynes. Measurements of Intake Separation Hysteresis in a Model Fan and Nacelle Rig. Journal of Propulsion and Power, 22(4):872-879, jul 2006 .

[6] Y. Colin, B. Aupoix, F. Boussuge, and P. Chanez. Numerical Simulation of the Distortion Generated by Crosswind Inlet Flows. International Soc. for Air Breathing Engines, pages 1-13, 2007.

[7] J. M. Delery. Shock Wave/Turbulent Boundary Layer Interaction and its Control. Progress in Aerospace Sciences, 22:209-280, 1985.

[8] A. Hadjadj and J.-P. Dussauge. Shock wave boundary layer interaction. Shock Waves, 19(6):449-452, nov 2009.

[9] J. Delery and J. Marvin. Shock-Wave Boundary Layer Interactions. Advisory Group for Aerospace Research and Development (AGARD), (21), 1986.

[10] N. A. Adams. Direct simulation of the turbulent boundary layer along a compression ramp at $\mathrm{M}=3$ and $\operatorname{Re} \theta=1685$. Journal of Fluid Mechanics, 420:47-83, 2000 .

[11] B. Becker, M. Reyer, and M. Swoboda. Steady and unsteady numerical investigation of transitional shock-boundary-layer-interactions on a fan blade. Aerospace Science and Technology, 11(7-8):507-517, nov 2007.

[12] T. S. Lund, X. Wu, and K. D. Squires. Generation of Turbulent Inflow Data for Spatially-Developing Boundary Layer Simulations. Journal of Computational Physics, 140(2):233-258, 1998. 
[13] G. Eitel-Amor, R. Örlü, and P. Schlatter. Simulation and validation of a spatially evolving turbulent boundary layer up to $\mathrm{Re}=8300$. International Journal of Heat and Fluid Flow, 47:57-69, 2014.

[14] D. Greenblatt, K. B. Paschal, C. S. Yao, J. Harris, N. W. Schaeffler, and A. E. Washburn. Experimental Investigation of Separation Control Part 1: Baseline and Steady Suction. AIAA Journal, 44(12):2820-2830, dec 2006.

[15] T. Makuni. Shock-Wave/Boundary-Layer Interactions in Transonic Aircraft Engine Intakes at High Incidence. PhD thesis, University of Cambirdge, 2017.

[16] P. G. Tucker. Unsteady Computational Fluid Dynamics in Aeronautics. Springer, 2014.

[17] Rolls-Royce. Rolls-Royce shares next generation engine designs, 2014.

[18] C. T.Wakelam, T. P. Hynes, H. P. Hodson, S. W. Evans, and P. Chanez. Separation Control for Aeroengine Intakes, Part 1: Low-Speed Investigation of Control Strategies. Journal of Propulsion and Power, 28(4):758-765, jul 2012.

[19] U. Oriji. Numerical Investigation of Intake Flows in Crosswind. PhD thesis, University of Cambridge, 2014.

[20] H. Babinsky and J. K. Harvey. Shock Wave Boundary-Layer Interaction. Cambridge University Press, 2011.

[21] T. E. Makuni, H. Babinsky, M. Slaby, and C. Sheaf. Experimental Investigations of Shock-Wave / Boundary-Layer Interactions in Transonic Aircraft Engine Intakes at High Incidence. 53rd AIAA Aerospace Sciences Meeting, (January):1-8, 2015.

[22] T. Makuni. Experimental Investigations of the Interaction between Normal Shock Waves and Transitional Boundary-Layers in Subsonic Intakes at High Incidence. PhD thesis, University of Cambridge, 2013.

[23] A. Coschignano, H. Babinsky, C. Sheaf, and E. Platt. Influence of near-leading edge curvature on the performance of aero-engine intake lips at high-incidence. In AIAA SciTech, pages 1-13, 2016.

[24] A. Coschignano, H. Babinsky, C. Sheaf, and E. Platt. Normal Shock WaveTurbulent Boundary Layer interactions in transonic intakes at incidence. In $A I A A$ SciTech, pages 1-16, 2018.

[25] D. P. Aeschliman and W. L. Oberkampf. Experimental Methodology for Computational Fluid Dynamics Code Validation. AIAA Journal, 36(5), 1998.

[26] C. T. Wakelam, T. P. Hynes, H. P. Hodson, S. W. Evans, and P. Chanez. Separation Control for Aero Engine Intakes, Part 2: High-Speed Investigations. Journal of Propulsion and Power, 28(4):766-772, jul 2012.

[27] B. E. Launder and W. P. Jones. On the Prediction of Laminarisation. Her Majesty's Stationary Office, (1036), 1969. 
[28] W. Kays, R. Loyd, and R. Moffat. The Turbulent Boundary Layer on a Porous Plate- An Experimental Study of the Fluid Dynamics with Strong Favorable Pressure Gradients and Blowing. Thermosciences Div. Dep. Mech. Eng. Stanford Univ. Rep. HMT-13., 1970.

[29] B. E. Launder and H. Stinchcombe. Non-Normal Similar Boundary Layers. Imp. Coll. Mech. Engng Dept. Rep. TWF/TN/21., 1976.

[30] S. Karimisani, U. R. Oriji, and P. G. Tucker. RANS Modelling of Accelerating Boundary Layers. Proceedings of the ASME 2013 International Mechanical Engineering Congress \& Exposition, pages 1-12, 2013.

[31] C. L. Rumsey and P. R. Spalart. Turbulence Model Behavior in Low Reynolds Number Regions of Aerodynamic Flowfields. AIAA Journal, 47(4):982-993, apr 2009 .

[32] Q. Zhang and Y. Yang. A new simpler rotation/curvature correction method for Spalart-Allmaras turbulence model. Chinese Journal of Aeronautics, 26(2):326333, apr 2013.

[33] P. R. Spalart and M. Shur. On the Sensitization of Turbulence Models to Rotation and Curvature. Aerospace Science and Technology, (5):297-302, 1997.

[34] M. L. Shur, M. K. Strelets, and A. K. Travin. Turbulence Modeling in Rotating and Curved Channels: Assessing the Spalart - Shur Correction. AIA A Journal, $38(5), 2000$.

[35] R. Watson. Large Eddy Simulation of Cutback Trailing Edges for Film Cooling Turbine Blades. PhD thesis, 2013.

[36] P. R. Spalart, W. H. Jou, M. K. Strelets, and S. R. Allmaras. Comments on the feasibility of LES for wings and on a hybrid RANS/LES approach. Advances in DNS/LES, 1(JANUARY):4-8, 1997.

[37] L. Davidson and S. H. Peng. Hybrid LES-RANS modelling: A one-equation SGS model combined with a k- $\omega$ model for predicting recirculating flows. International Journal for Numerical Methods in Fluids, 43(9):1003-1018, 2003.

[38] D. L. Motycka. Reynolds Number and Fan / Inlet Coupling Effects on Subsonic Transport Inlet Distortion. Journal of Propulsion and Power, 1(3):229-234, 1985.

[39] J. P. Longley and E. M. Greitzer. Inlet distortion effects in aircraft propulsion system integration. In AGARD, Steady and Transient Performance Prediction of Gas Turbine Engines, 1992.

[40] T. Cao, N. R. Vadlamani, P. G. Tucker, A. R. Smith, M. Slaby, and C. T. J. Sheaf. Fan-Intake Interaction Under High Incidence. (49682):V001T01A014, 2016.

[41] A. Peters, Z. S. Spakovszky, W. K. Lord, and B. Rose. Ultra-Short Nacelles for Low Fan Pressure Ratio Propulsors. (45578):V01AT01A029, 2014. 
[42] T. Andrew, T. Lehnig, and S. Rahm. Inlet/fan cowl design for increased angle-ofattack requirements of transport aircraft - Methodology and test results. In 27 th Joint Propulsion Conference, Joint Propulsion Conferences. American Institute of Aeronautics and Astronautics, jun 1991.

[43] D. S. Dolling. Fifty Years of Shock-Wave / Boundary-Layer Interaction Research : What Next? 39(8):1517-1531, 2001.

[44] W. D. Bachalo and D. A. Johnson. Transonic, Turbulent Boundary-Layer Separation Generated on an Axisymmetric Flow Model. AIAA, 24(3):437-443, 1986.

[45] J. Ackeret, F. Feldmann, and N. Rott. Investigations of Compression Shocks and Boundary Layers in Gases Moving at High Speed. Technical Memorandom, NACA, 10(1113), 1947.

[46] H. W. Liepmann. The Interaction Between Boundary Layer and Shock Waves in Transonic Flow. Journal of the Aeronautical Sciences (Institute of the Aeronautical Sciences), 13(12):623-637, dec 1946.

[47] A. Zheltovodov. Shock waves/turbulent boundary-layer interactions - Fundamental studies and applications. In Fluid Dynamics Conference, Fluid Dynamics and Co-located Conferences. American Institute of Aeronautics and Astronautics, jun 1996.

[48] D. Knight and G. Degrez. Shockwaveboundarylayer interactions in high Mach number flows - a critical survey of current CFD prediction capabilities. AGARD AR-319, 2, 1998.

[49] D. Knight, H. Yan, A. G. Panaras, and A. Zheltovodovc. Advances in CFD prediction of shock wave turbulent boundary layer interactions. Progress in Aerospace Sciences, 39(2-3):121-184, feb 2003.

[50] R. V. Kaenel, L. Kleiser, N. a. Adams, and J. B. Vos. Large-Eddy Simulation of Shock-Turbulence Interaction. AIAA Journal, 42(12):2516-2528, dec 2004.

[51] E. Garnier, P. Sagaut, and M. Deville. Large Eddy Simulation of Shock/Boundary-Layer Interaction. AIAA Journal, 40(10):1935-1944, oct 2002.

[52] U. Piomelli. Wall-layer models for large-eddy simulations. Progress in Aerospace Sciences, 44(6):437-446, 2008.

[53] H. Loyau, P. Batten, and M. A. Leschziner. Modelling Shock / Boundary-Layer Interaction with Nonlinear Eddy-Viscosity Closures. Flow, Turbulence and Combustion, 60:257-282, 1998.

[54] J. Delery, C. Copy, and J. Reisz. Analyse au velocimetre laser bidirectionnel d'une interaction choc-couche limite turbulente avec decollement etendu. ONERA Report No. 37/7078 AY 014, 1980.

[55] D. D. Apsley and M. A. Leschziner. A new low-Reynolds-number nonlinear twoequation turbulence model for complex ${ }^{-}$ows. In, 19:209-222, 1998. 
[56] A. Jameson, W. Schmidt, and E. Turkel. Numerical solution of the Euler equations by finite volume methods using Runge Kutta time stepping schemes. In 14th Fluid and Plasma Dynamics Conference, Reston, Virigina, jun 1981. American Institute of Aeronautics and Astronautics.

[57] F. Ducros, V. Ferrand, F. Nicoud, C. Weber, D. Darracq, C. Gacherieu, and T. Poinsot. Large-Eddy Simulation of the Shock/ Turbulence Interaction. Journal of Computational Physics, 152:517-549, 1999.

[58] E. Garnier, P. Sagaut, and M. Deville. A Class of Explicit ENO Filters with Application to Unsteady Flows. Journal of Computational Physics, 170:184-204, 2001.

[59] A. Jameson. The Present Status, Challenges, and Future Developments in Computational Fluid Dynamics. In 12th Australasian Conference on Fluid Mechanics, 1995 .

[60] Z. R. Murphree, J. Jagodzinski, E. S. Hood, N. T. Clemens, and D. S. Dolling. Experimental Studies of Transitional Boundary Layer Shock Wave Interactions. In 44th AIAA Aerospace Sciences Meeting and Exhibit, number January, pages $1-10,2006$.

[61] Lander and Sandham. Closure Strategies for Turbulent and Transitional Flows. Cambridge University Press, 2002.

[62] O. Reynolds. On the dynamic theory of incompressible viscous fluids and the determination of the criterion. Philosophical transactions. Series A, Mathematical, physical, and engineering sciences, 186:123-164, 1895.

[63] E. Mastorakos. 4A12: Turbulence and Vortex Dynamics, Lecture Notes, 2014.

[64] P. R. Spalart and S. R. Allmaras. A one-equation turbulence model for aerodynamic flows. La Recherche Aerospatiale, 1:5-21, 1994.

[65] M. Shur, M. Strelets, L. Zajkov, A. Gulyaev, V. Kozlov, and A. Sekundov. Comparative numerical testing of one- and two-equation turbulence models for flows with separation and reattachment. In 33rd Aerospace Sciences Meeting and Exhibit, Aerospace Sciences Meetings. American Institute of Aeronautics and Astronautics, jan 1995.

[66] E. R. V. Driest. On Turbulent Flow Near a Wall. Journal of the Aeronautical Sciences (Institute of the Aeronautical Sciences), 23(11):1007-1011, nov 1956.

[67] J. Smagorinsky. General Circulation Experiments With the Primitive Equations. Monthly Weather Review, 91(3):99-164, 1963.

[68] D. K. Lilly. On the application of the eddy viscosity concept in the Inertial subrange of turbulence. Boulder Colo.: National Center for Atmospheric Research, (January), 1966.

[69] F.Nicoud and F. Ducros. Subgrid scale stress modelling based on the squared of the velocity gradient tensor. pages 1-36, 1999. 
[70] F. Nicoud, H. B. Toda, O. Cabrit, S. Bose, and J. Lee. Using singular values to build a subgrid-scale model for large eddy simulations. Physics of Fluids, 23(085106), 2011.

[71] L. Lapworth. HYDRA CFD: A Framework for Collaborative CFD Development. In Proceedings of the 2nd International Conference on Scientific and Engineering Computation (IC-SEC 2004), Singapore, 2004.

[72] P. L. Roe. Approximate Riemann Solvers, Parameter Vectors, and Difference Schemes. Journal of Computational Physics, 43:357-372, 1981.

[73] C. Rumsey, T. Gatski, W. Sellers, V. Vatsa, and S. Viken. Summary of the 2004 CFD Validation Workshop on Synthetic Jets and Turbulent Separation Control. 2nd AIAA Flow Control Conference, pages 1-45, jun 2004.

[74] T. Gatski and C. Rumsey. Case 3, Langley Research Center Workshop, 2004.

[75] P. E. Morgan, D. P. Rizzetta, and M. R. Visbal. Large-Eddy Simulation of Separation Control for Flow over a Wall-Mounted Hump. AIAA Journal, 45(11):26432660, nov 2007.

[76] A. Avdis, S. Lardeau, and M. Leschziner. Large Eddy Simulation of Separated Flow over a Two-dimensional Hump with and without Control by Means of a Synthetic Slot-jet. Flow, Turbulence and Combustion, 83(3):343-370, may 2009.

[77] J. a. Franck and T. Colonius. Compressible Large-Eddy Simulation of Separation Control on a Wall-Mounted Hump. AIAA Journal, 48(6):1098-1107, jun 2010.

[78] B. D. You, M. Wang, and P. Moin. Large-eddy simulation of flow over a wallmounted hump with separation control. pages 223-234, 2005.

[79] G. I. Park. Wall-modeled large-eddy simulation of a separated flow over the NASA wall-mounted hump. Center for Turbulence Research Annual Research Briefs 2015, (2006), 2016.

[80] V. Krishnan, K. D. Squires, and J. R. Forsythe. Prediction of Separated Flow Characteristics over a Hump using RANS and DES. In 2nd AIAA Flow Control Conference, number July, 2004.

[81] C. L. Rumsey and D. Greenblatt. Parametric Study of Flow Control Over a Hump Model Using an Unsteady Reynolds- Averaged Navier-Stokes Code. (September), 2007.

[82] C. L. Rumsey and D. Greenblatt. Flow Control Predictions Using Unsteady Reynolds-Averaged Navier-Stokes Modeling: A Parametric Study. AIA A Journal, 47(9):2259-2262, sep 2009.

[83] D. C. Lyons, F. J. Zajaczkowski, L. J. Peltier, and E. G. Paterson. Assessment of DES Models for Separated Flow from a Hump in a Turbulent Boundary Layer. In Proceedings of the 5th Joint ASME/JSME Fluids Engineering Conference, number 2004, pages 1-10, 2007. 